\title{
Synthesis and characterization of molecularly doped tri-p- tolylamine/polycarbonate blends using solid-state NMR
}

\author{
Brian D. Kesling \\ West Virginia University
}

Follow this and additional works at: https://researchrepository.wvu.edu/etd

\section{Recommended Citation}

Kesling, Brian D., "Synthesis and characterization of molecularly doped tri-p-tolylamine/polycarbonate blends using solid-state NMR" (2004). Graduate Theses, Dissertations, and Problem Reports. 2116.

https://researchrepository.wvu.edu/etd/2116

This Dissertation is protected by copyright and/or related rights. It has been brought to you by the The Research Repository @ WVU with permission from the rights-holder(s). You are free to use this Dissertation in any way that is permitted by the copyright and related rights legislation that applies to your use. For other uses you must obtain permission from the rights-holder(s) directly, unless additional rights are indicated by a Creative Commons license in the record and/ or on the work itself. This Dissertation has been accepted for inclusion in WVU Graduate Theses, Dissertations, and Problem Reports collection by an authorized administrator of The Research Repository @ WVU.

For more information, please contact researchrepository@mail.wvu.edu. 
Synthesis and Characterization of Molecularly Doped Tri-pTolylamine/Polycarbonate Blends Using Solid-State NMR

\author{
Brian D. Kesling
}

Dissertation submitted to the Eberly College of Arts and Science at West Virginia University in partial fulfillment of the requirements

for the degree of

Doctor of Philosophy in

Physical Chemistry

Terry Gullion, Ph.D., Chair

Kenneth Showalter, Ph.D.

Charles Jaffe, Ph.D.

Bjorn Soderberg, Ph.D.

David Murray, Ph.D.

Department of Chemistry, Morgantown, WV 


\section{Abstract \\ Synthesis and characterization of molecularly doped tri-p-tolylamine/ \\ polycarbonate blends using solid-state NMR. \\ Brian D. Kesling}

Polymers doped with organic photoconductors play an important role in the xerography process. The macroscopic distribution of the doped polymers has been assumed to follow a uniform distribution model as a result of the lack of information regarding the spatial relationships or macroscale organization of the dopant molecules. Because of the amorphous nature of the blend, characterization of the dopant molecule organization is unattainable by the traditional method of x-ray diffraction. Solid-state NMR experiments begun by Hewitt et al. have been expanded using two modern solid-state NMR techniques: Rotational Echo, Double Resonance (REDOR) and Rotational Echo, Adiabatic

Passage Double Resonance (REAPDOR). These techniques, coupled with isotope labeling syntheses, provide data that suggests a high degree of macrosopic organization in the dopant molecules and a model for the organization is proposed. 


\section{Table of Contents}

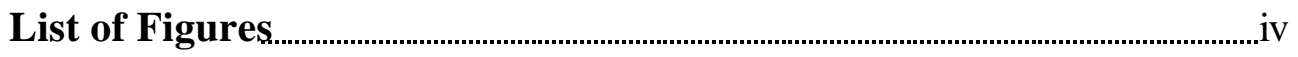

\section{Chapter}

1 Introduction $\ldots$

2 Solid-State NMR

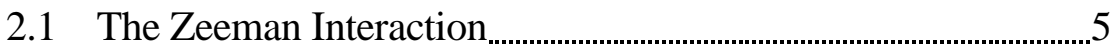

2.2 Chemical Shift Anisotropy

2.3 Heteronuclear Dipole-dipole Interaction ............................................

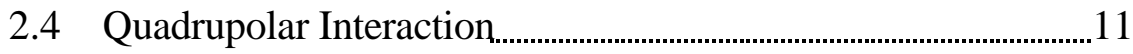

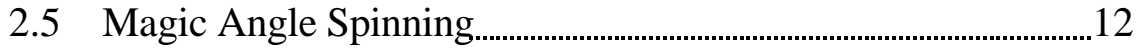

2.6 Rotational-Echo, Double-Resonance NMR ………………………....14

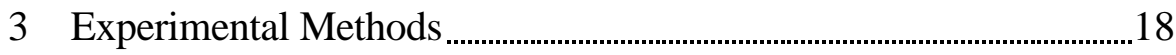

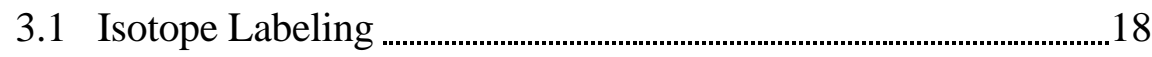

3.1.1 TTA Synthesis ........................................................................................... 19

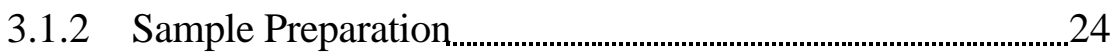

3.2 NMR

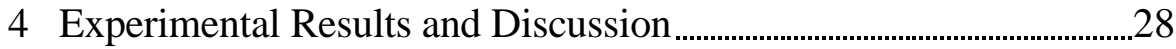

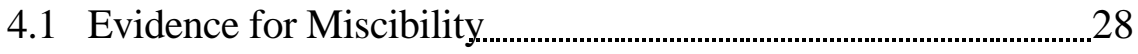

4.2 Structural Relationship Between TTA Molecules...............................35

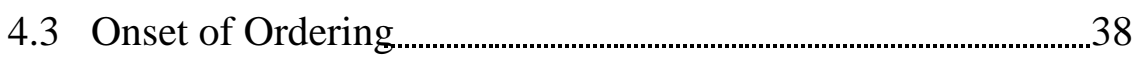

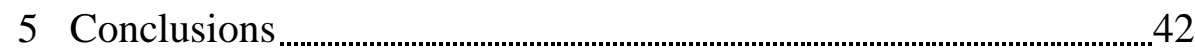

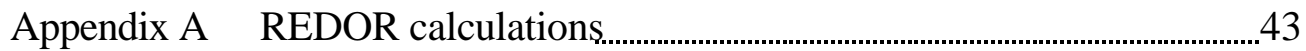

Appdenix B NMR spectra of synthesized compounds $\quad$.......................................... 44

Bibliography 


\section{List of Figures}

Figure 1.1 The xerography process

Figure 1.2 Electron hopping in a photoreceptor

Figure 1.3 Commonly used organic photoconductors

Figure 2.1.1 Motion of $\mu$ in a magnetic field

Figure 2.1.2 Zeeman interaction for $\mathrm{I}=1 / 2$

Figure 2.2.1 Shielding tensor effect on ${ }^{13} \mathrm{C}$ resonance of ethene

Figure 2.2.2 Simulated ${ }^{13} \mathrm{C}$ NMR powder spectrum of ethene

Figure 2.3.1 Effect of S-spin on I-spin spectrum for $\mathrm{S}=1 / 2$ and $\mathrm{I}=1 / 2$

Figure 2.3.2 Static powder spectrum for $\mathrm{S}=1 / 2$ spin coupled to $\mathrm{I}=1 / 2$

Figure 2.4.1 Effect of Quadrupole interaction for $\mathrm{S}=1$

Figure 2.4.2 Quadrupole powder spectrum for an $\mathrm{S}=1$ nucleus

Figure 2.5.1 A solid-state NMR rotor spinning at the magic angle

Figure 2.5.2 MAS effect on the NMR spectrum of ${ }^{31} \mathrm{P}$ in solid triphenylphosphine

Figure 2.6.1 Three orientation dependent resonances

Figure 2.6.2 S-spin effects on the $\mathrm{H}_{\mathrm{L}}$ of a nearby I-spin

Figure 2.6.3 The REDOR experiment

Figure 2.6.4 Theoretical REDOR plot for a dipole-dipole interaction

Figure 3.1.1 Sythesis of tri-p-tolylamine (TTA)

Figure 3.2.1 Schematic of the NMR spectrometer

Figure 4.1.1 The PC monomer and TTA molecule with ${ }^{13} \mathrm{C}$ positions labeled

Figure 4.1.2 ${ }^{13} \mathrm{C}$ spectra showing ${ }^{13} \mathrm{C}$ peak positions of PC and TTA

Figure 4.1.3 Full and reduced ${ }^{13} \mathrm{C}-{ }^{2} \mathrm{H}$ REDOR spectra for a $\mathrm{PC} / \mathrm{d}_{21}$-TTA blend

Figure 4.1.4 $\Delta \mathrm{S} / \mathrm{S}$ REDOR data for methyl ${ }^{13} \mathrm{C}$ of TTA

Figure 4.1.5 ${ }^{13} \mathrm{C}_{-}{ }^{14} \mathrm{~N}$ REAPDOR for PC/TTA Blend

Figure 4.1.6 Cartoon representation of Hewitt's stacking model

Figure $4.2 .1{ }^{13} \mathrm{C}^{15} \mathrm{~N}$ REDOR data measuring TTA to TTA proximity 
Figure 4.2.2 ${ }^{13} \mathrm{C}^{-15} \mathrm{~N}$ REDOR data measuring distance from a TTA ${ }^{13} \mathrm{C}$ methyl group to a neighboring TTA ${ }^{15} \mathrm{~N}$

Figure 4.2.3 ${ }^{13} \mathrm{C}^{-15} \mathrm{~N}$ REDOR data for a series of blends

Figure 4.2.4 TTA crystal lattice along the c-axis

Figure 4.2.5 Ring carbon to nearest nitrogen distances in pure TTA

Figure 4.3.1 REDOR data for blends with differing weight percents

Figure 4.3.2 Possible TTA chain arrangements in PC

Figure 4.3.3 REDOR data from a blend of deuterated PC and TTA 


\section{Chapter 1. Introduction}

Xerography has grown into a process responsible for upwards of 5 billion copies per day with an annual revenue in excess of $\$ 100$ billion. The process of xerography requires the formation of a charge pattern that replicates the desired image and is illustrated in Figure 1. In Step (1), a corona discharge places a uniform positive charge on the

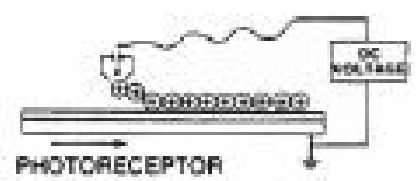

(1) Charge

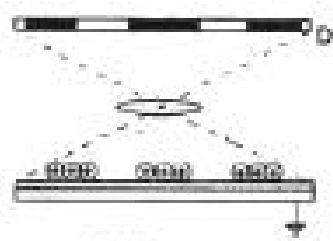

(2) Expose

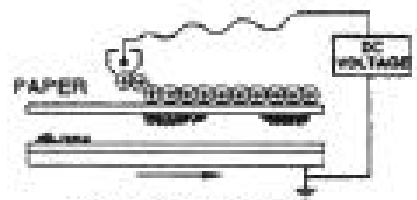

(4) Transfer

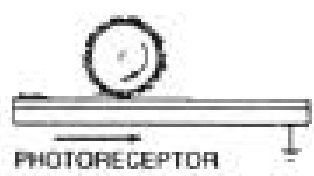

(6) Clean

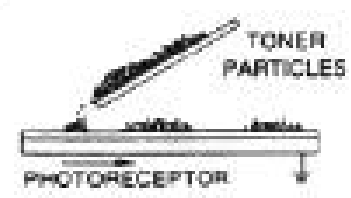

(3) Develop

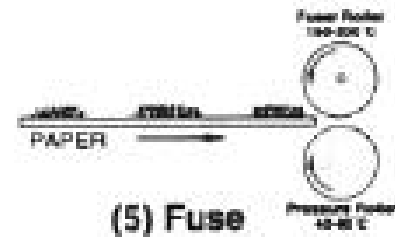

(5) Fuse

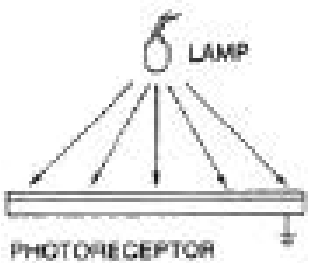

(7) Erase
Figure 1.1. The 7 Step process of xerography ${ }^{\text {. }}$. Organic photoconductors play an important role in (2).

photoreceptor surface, typically composed of a polymer film doped with a photo-organic molecule. Step (2) is the exposure of the photoreceptor surface to an optical image being reproduced and the formation of a latent image. Step (3) consists of charged toner particles being brought into contact with the latent image. The toner particles are transferred to a receiver, such as paper, in Step (4) and fused in Step (5). Steps (6) and (7) involve the removal of the remaining toner particles and uniform exposure of the photoreceptor to 
remove any remaining surface charges. The entire process can be replicated quickly enough to generate more than 100 pages per minute.

Figure 2 illustrates the process by which the latent image is formed during Step (2). A typical photoreceptor consists of a polymer film ${ }^{2}$ (boundaries indicated by vertical lines) doped with organic photoconducting molecules. Common photoconducting molecules contain $\mathrm{sp}^{3}$-hybridized nitrogen sites with easily photoionized lone pair electrons. Incident light photoexcites electrons, releasing them to neutralize the positive charge deposited on the photoreceptor surface (left side of Figure 2), thereby forming the latent image. An electric field is applied across the photoreceptor to drive the electron hopping process. The right side of the photoreceptor film is grounded so that photo-generated holes can be neutralized.

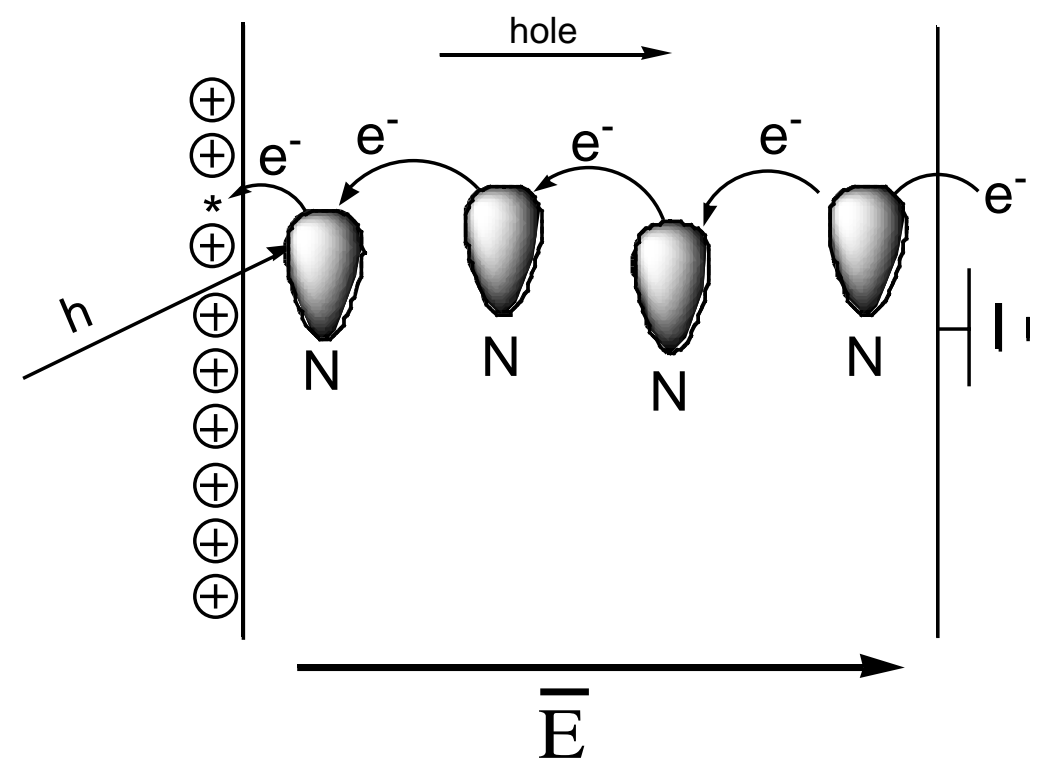

Figure 1.2. The vertical lines represent the edges of the molecularly doped polymer film. An electric field, $\bar{E}$, is applied across the sample. A photoexcited electron neutralizes a surface charge to create an image. The $\bar{E}$ drives the photoexcited etoward the charged surface. The ground plane provides a source of $e^{-}$to neutralize the holes produced after photoexcitation

Most modern copiers use a miscible blend consisting of an organic photoconducting dopant molecule blended in a polycarbonate matrix ${ }^{2}$ for the image forming 
step. Polycarbonate is chosen because it forms clear and durable films. The dopant molecule may be either a hole transporting or an electron transporting molecule.

Figure 3 shows a variety of charge transporting molecules that have been or are currently being used in the xerography process. Each compound has one or more $\mathrm{sp}^{3}$ hybridized nitrogen atoms. The $\mathrm{N}-\mathrm{C}$ bonds in these molecules are very nearly planar, and each of those nitrogens has a lone pair of electrons available for photoexcitation.
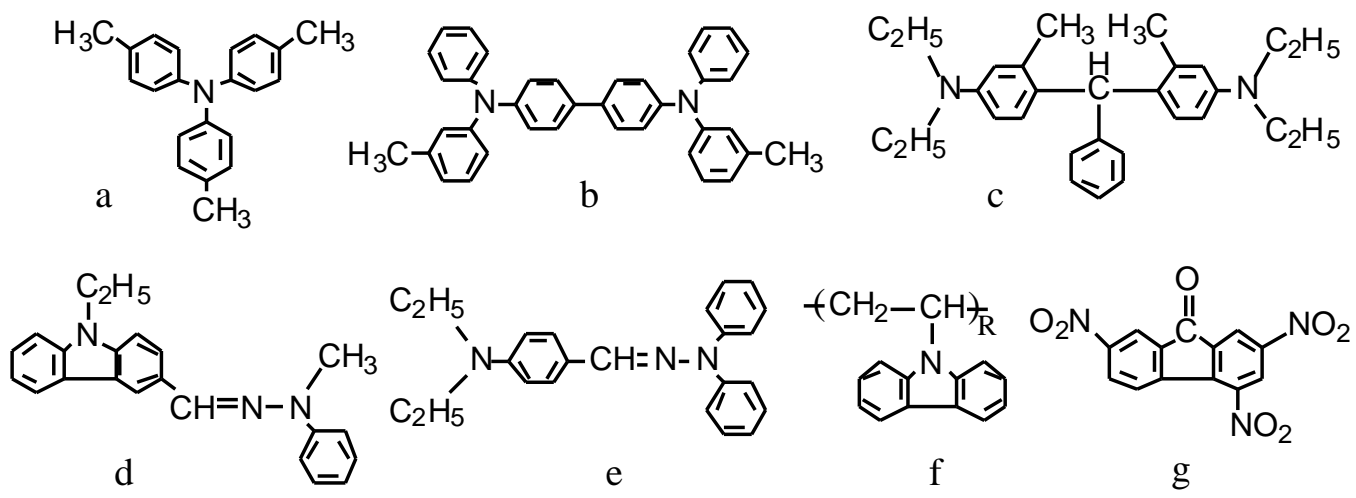

Figure 1.3. Molecules commonly used in the xerography process today ${ }^{I}$ a) trip-tolylamine, $b$ ) biphenyldiamine, c) bisdiethylamino triphenylmethane, $d$ carbazole hydrazone, e) benzaldehyde hydrazone, $f)$ poly( $N$-vinyl carbazole), and g) 2,4,7-trinitro-9-fluorenone

The dopant molecules play a central role in the xerography process, yet there is little information available about how they are ordered in the polycarbonate (PC) matrix. Generally, it is assumed that no long-range order of dopant molecules is present in miscible molecularly doped polymer blends. The structural relationship between dopant molecules is crucial to the understanding of the electron hopping process that occurs after photoexcitation. Because of its relatively simple structure, tri-p-tolylamine (TTA) is a hole transport molecule that is often used in studies of blends for the xerography process. A major problem with determining the TTA spatial positions results from the fact that the blend is an amorphous solid and traditional methods used to obtain structural information typically require the sample to be in liquid form (liquid NMR) or a crystalline solid (x-ray diffraction). There are modern $\mathrm{x}$-ray techniques that can provide information about materials lacking long-range order, but the nature of the x-ray interaction requires both a 
contrast in the weights of the atoms being studied and that the atoms have mass equal to or greater than carbon. While the TTA molecules and PC monomers contain carbon atoms, neither TTA molecules nor PC monomers contain sufficiently different heavy molecules to distinguish one from the other. Because of the difficulty associated with characterizing these polymer blends, most theoretical models used to calculate hole transport simply assume that the charge transporting molecules are arranged by uniform dispersion. Solid-state nuclear magnetic resonance (SSNMR) experiments are capable of providing long range structural information in systems lacking long-range order and can provide important structural information.

The goal of this work is to develop a model of the ordering of TTA on the molecular level of the TTA:PC blend. A model will be produced by combining judicious isotope labeling and SSNMR. If ordering of the dopant molecules can be proven, this work will have a major impact on polymer science since it is generally assumed that miscible blends lack any long-range order. 


\section{Chapter 2. Solid-State NMR}

NMR is a powerful tool used for chemical analysis and to determine molecular structures. Solution state NMR is familiar to chemists and biochemists. It is used to characterize small molecules and to determine structures of large molecules, such as proteins. Although less familiar to most chemists, solid-state NMR has recently become a routine method for determining molecular structures. The various interactions that must be dealt with in solid-state NMR are described in this section.

The internal NMR interactions present in the blends are the chemical shift anisotropy, quadrupole interaction, and dipole-dipole interactions. The dipole-dipole interaction is of particular interest since it contains distance information. However, the dipole-dipole interaction for these blends is two to four orders of magnitude smaller than the other interactions. Yet, it is still possible to use this weak interaction to obtain important distance information.

\subsection{The Zeeman interaction}

In order for a nucleus to be studied by NMR, it must possess both angular

momentum, $\vec{J}$, and a magnetic moment $\vec{\mu} .^{3} \vec{J}$ and $\vec{\mu}$ are vectors and are related by $\vec{\mu}=\gamma \vec{J}$, where $\gamma$ is the gyromagnetic ratio. In the presence of an externally applied magnetic field $\overrightarrow{B_{0}}$, a magnetic moment experiences a torque $\vec{\tau}$ such that $\vec{\tau}=\overrightarrow{\mu \times} \overrightarrow{B_{0}}$. The angular momentum can be related to the torque through the classical equation of motion $\vec{\tau}=\frac{d \vec{J}}{d t}$. Combining the two torque equations results in $\frac{d \vec{\mu}}{d t}=\vec{\mu} \times\left(\gamma \overrightarrow{B_{0}}\right)$, which describes the precession of the magnetic moment about $\vec{B}_{0}$ at a rate $\omega_{0}$ equal to $\gamma B_{0}$. Figure 2.1.1 shows the precession of $\vec{\mu}$ about $\overrightarrow{B_{0}}$. If $\vec{\mu}$ is placed inside the volume defined by a solenoidal coil 
of wire, then the precessional motion of $\vec{\mu}$ induces a voltage in the coil. This induced voltage is the source of all NMR signals.

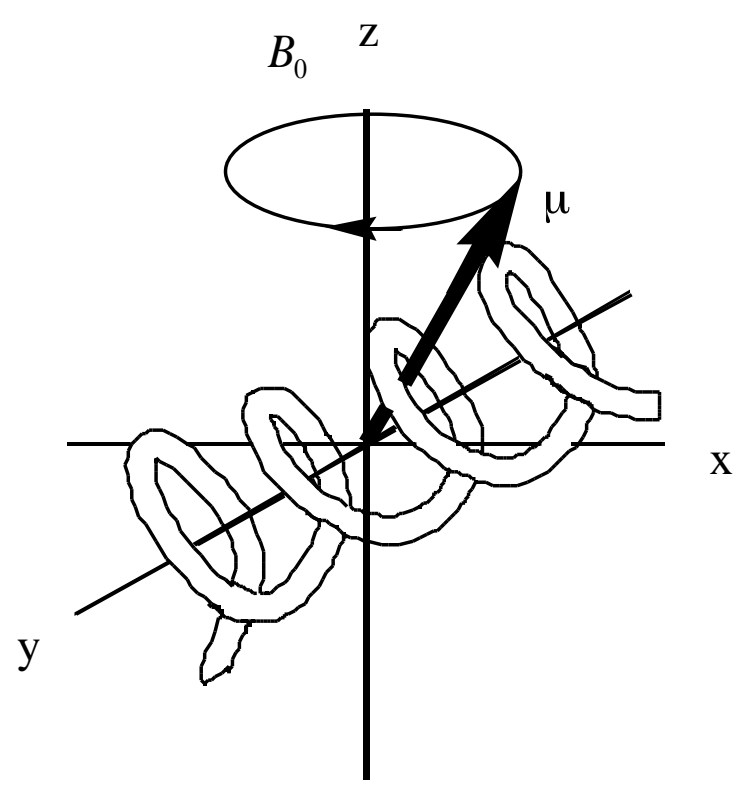

Figure 2.1.1. Precessional motion of $\vec{\mu}$ in a magnetic field $\vec{B}$.

The energy between a magnetic moment and an external magnetic field is described by the Hamiltonian

$$
\mathcal{H}_{o}=-\vec{\mu} \bullet \overrightarrow{B_{0}}
$$

A dimensionless angular momentum operator $\vec{I}$ is defined through

$$
\vec{J}=\hbar \vec{I}
$$

$I$ is the nuclear spin quantum number and has either integer or half-integer values. The eigenvalue of $I^{2}$ is $I(I+1)$ and $I_{z}$ has eigenvalues ranging from $-I$ to $I$ in integer increments.

The Hamiltonian of an I-spin in a magnetic field $\vec{B}_{0}$ can now be given as $\mathcal{H}_{0}=-\gamma \hbar B_{0} I_{z}$ with energy eigenvalues $E_{m}=-\gamma \hbar B_{0} m$. The difference between adjacent energy states is $\Delta E=+\gamma \hbar B_{0}$. The interaction between the magnetic moment of the nucleus and the externally applied field is known as the Zeeman interaction and is illustrated in Figure 2.1.2 for $\mathrm{I}=1 / 2$. 


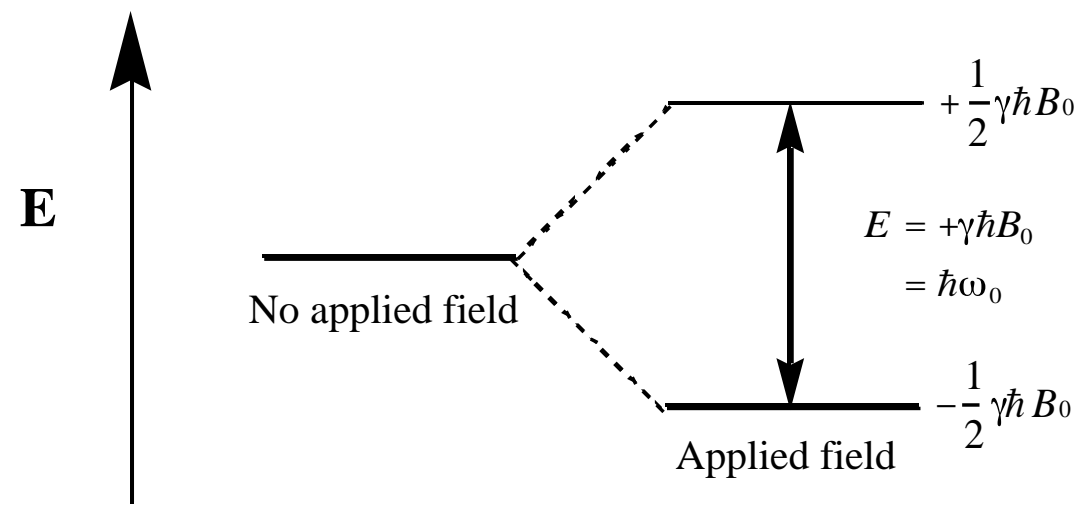

Figure 2.1.2. Zeeman interaction for $I=1 / 2$ nucleus in an applied field.

At thermal equilibrium, the energy states are populated according to the Boltzman distribution. It is the difference in the population that accounts for the bulk nuclear magnetization, $\vec{M} \cdot \vec{M}$ is proportional to $\vec{\mu}$ and obeys the same equation of motion as $\vec{\mu}$. For simplicity, NMR calculations are often performed in a rotating frame with a rotation rate of $\omega_{0}=\gamma B_{0}$ so that the nuclear magnetization appears static provided no internal interactions are present.

The presence of internal interactions is where NMR gains its usefulness. The chemical shift correlates specific resonances to functional groups, dipole-dipole interactions provide distance information, and quadrupole interactions provide information about molecular symmetry. Following is a discussion of the NMR interactions present in the blended systems.

\subsection{Chemical-Shift Anisotropy}

In addition to the large externally applied magnetic field produced by the NMR magnet, nuclei also experience smaller locally generated magnetic fields that arise from the motion of electrons in their molecular orbitals in response to the application of a strong externally applied magnetic field. Because the electron cloud surrounding a nucleus is rarely symmetric, this response leads to an orientation dependent first order perturbation of the 
energy levels. This perturbation is called chemical shift shielding because the small local magnetic field created by the electrons partially shields the nucleus from the large static field (in effect, this is Lenz's Law). The chemical shift contribution to the Hamiltonian is $\mathcal{H}_{C S}=\gamma \bar{I} \bar{I}^{=} \bar{\sigma} \bullet \bar{B}_{0},{ }^{3}$ where $\bar{\sigma}$ is the chemical shift shielding tensor. The effect of the shielding tensor on ${ }^{13} \mathrm{C}$ resonance positions for different orientations of ethene is illustrated in Figure 2.2.1. The position of the ${ }^{13} \mathrm{C}$ NMR resonance is different for each of the shown orientations.

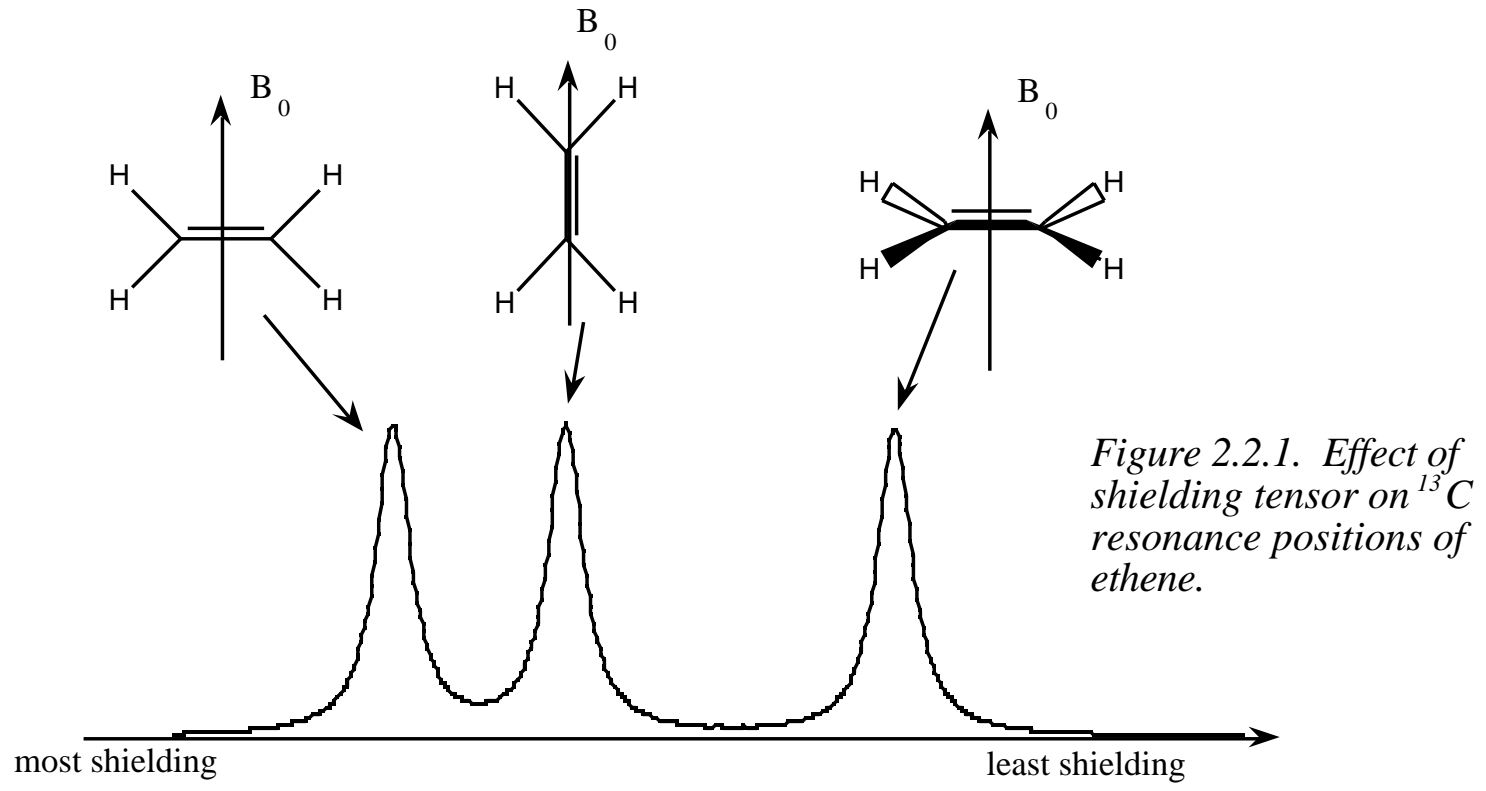

All molecular orientations with respect to $\vec{B}_{0}$ are present in a powder sample. Hence the chemical shift dominated powder spectrum has a wide range of resonances. In a suitable frame, $\sigma=\left(\begin{array}{ccc}\sigma_{11} & 0 & 0 \\ 0 & \sigma_{22} & 0 \\ 0 & 0 & \sigma_{33}\end{array}\right)$, and the average shift is $\sigma_{i s o}=1 / 3\left(\sigma_{11}+\sigma_{22}+\sigma_{33}\right)$. The corresponding resonance frequency for a shift tensor with Euler angles $\alpha$ and $\beta$ with respect to $\overrightarrow{B_{0}}$ is given by $\omega=\omega_{11} \sin \beta \cos \alpha+\omega_{22} \sin \beta \sin \alpha+\omega_{33} \cos \beta$ where $\omega_{j j}=-\gamma_{N} B_{0}\left(1-\sigma_{j j}\right)$. An example of a ${ }^{13} \mathrm{C}$ powder pattern is illustrated in Figure 2.2.2. 
Whereas ${ }^{13} \mathrm{C}$ resonances in liquids are on the order of a $\mathrm{Hz}$ in width, powder patterns in solid state NMR may span tens of kilohertz in frequency. In liquid samples, the molecules tumble rapidly and sample all molecular orientations. Consequently, the shielding interaction is averaged to $\sigma_{i s o}$ and the liquid NMR resonance position is $\gamma B_{0}\left(1-\sigma_{\text {iso }}\right)$.

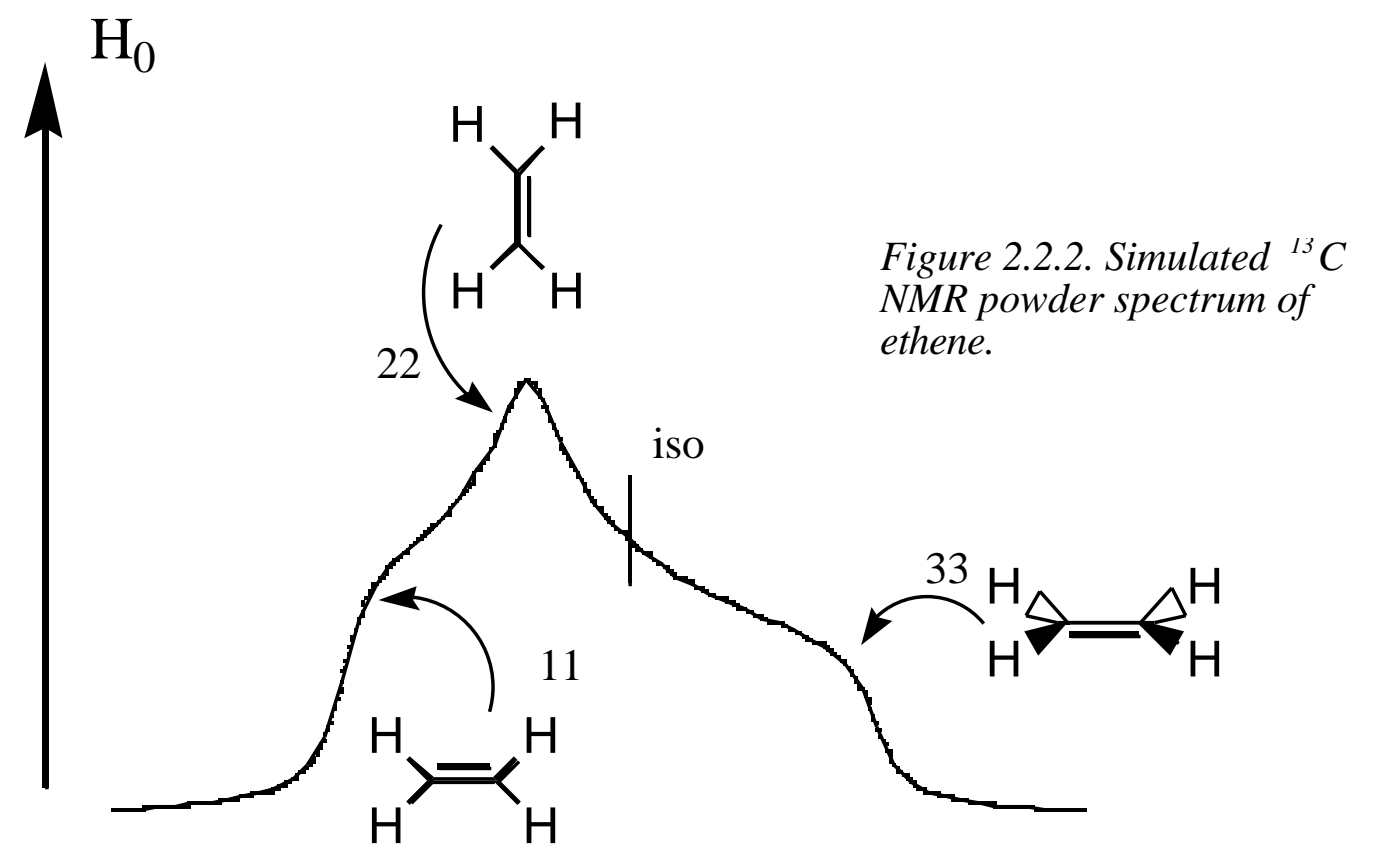

\subsection{Heteronuclear Dipole-dipole interaction}

The Hamiltonian for a pair of dipolar coupled spins $\overrightarrow{\mathrm{I}}$ and $\overrightarrow{\mathrm{S}}$ is $\mathcal{H}_{D}=\bar{I} \bullet \bar{D} \bullet \bar{S}$

where $^{3} \stackrel{=}{D}=\hbar \omega_{D}\left(\begin{array}{ccc}1-3 x^{2} & -3 x y & -3 x z \\ -3 x y & 1-3 y^{2} & -3 y z \\ -3 x z & -3 y z & 1-3 z^{2}\end{array}\right)$ and $\omega_{D}=\frac{\mu_{0} \gamma_{I} \gamma_{S} \hbar}{4 \pi r^{3}}$

$\gamma_{I}$ and $\gamma_{S}$ are the respective magnetogyric ratios and $r$ is the distance between the two nuclei. Replacing the Cartesian coordinates with spherical coordinates allows the Hamiltonian to be rewritten as $\mathcal{H}_{D}=\hbar \omega_{D} \mathrm{~A}$, where $\mathrm{A}=\left(1-3 \cos ^{2} \theta\right) I_{z} S_{z}$ for a heteronuclear spin pair. The observed I-spins will experience two local fields when coupled 
to an S-spin: one due to the $m_{S}=+1 / 2$ spin state and one due to the $m_{S}=-1 / 2$ spin state. The effect of the local fields is to split the single resonance from the Zeeman only interaction to a pair of resonances. This effect is illustrated in Figure 2.3.1.

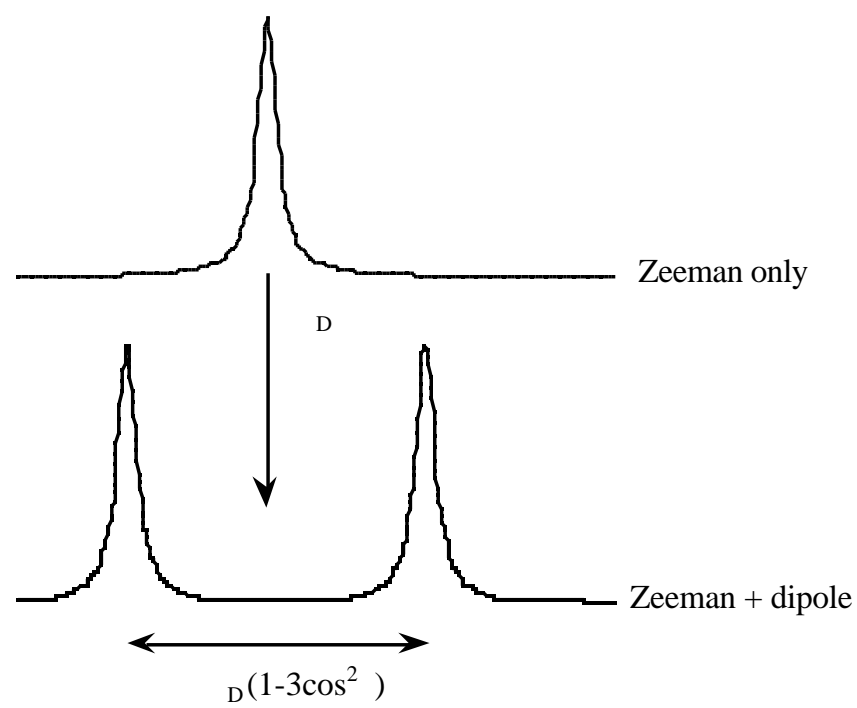

Figure 2.3.1. Effect of spin$S$ on the spectrum of spin-I, where $I=1 / 2$ and $S=1 / 2$

The static powder spectrum of the I-spin coupled to an $S=1 / 2$ spin is generated by summing over the angular dependent resonance frequency $\pm \frac{1}{2} \omega_{D}\left(1-3 \cos ^{2} \theta\right)$ over all values of $\theta$, and weighting each resonance position by $\sin \theta$. A powder spectrum for an $S=1 / 2$ nucleus coupled to an $I=1 / 2$ nucleus is shown in Figure 2.3.2. The difference in the peak heights is the dipole coupling $\omega_{D}$. The dipole coupling has special significance in structure studies of

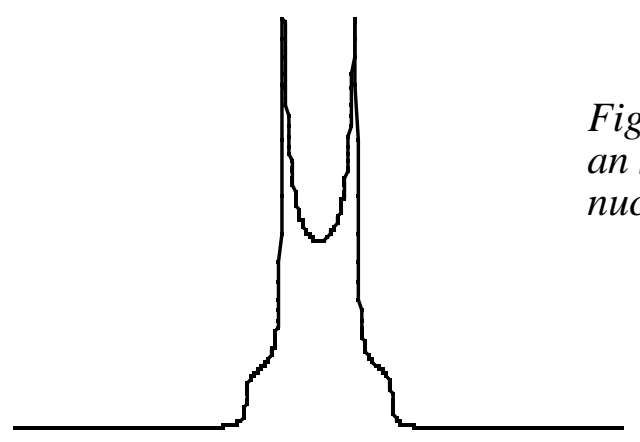

Figure 2.3.2 Static powder spectrum for an $S=1 / 2$ nucleus coupled to an $I=1 / 2$ nucleus. 
materials since $\omega_{D}=\frac{\mu_{0} \gamma_{I} \gamma_{S} \hbar}{4 \pi r^{3}}$. Hence, measuring $\omega_{D}$ provides the distance between the Ispin nucleus and the S-spin nucleus.

\subsection{Quadrupolar Interaction (S=1)}

S>1/2 nuclei have a quadrupole moment which can interact with electric field gradients generated by local electronic structure ${ }^{3}$. The quadrupole interaction shifts the Zeeman energy levels as illustrated in the following Figure for $S=1$.

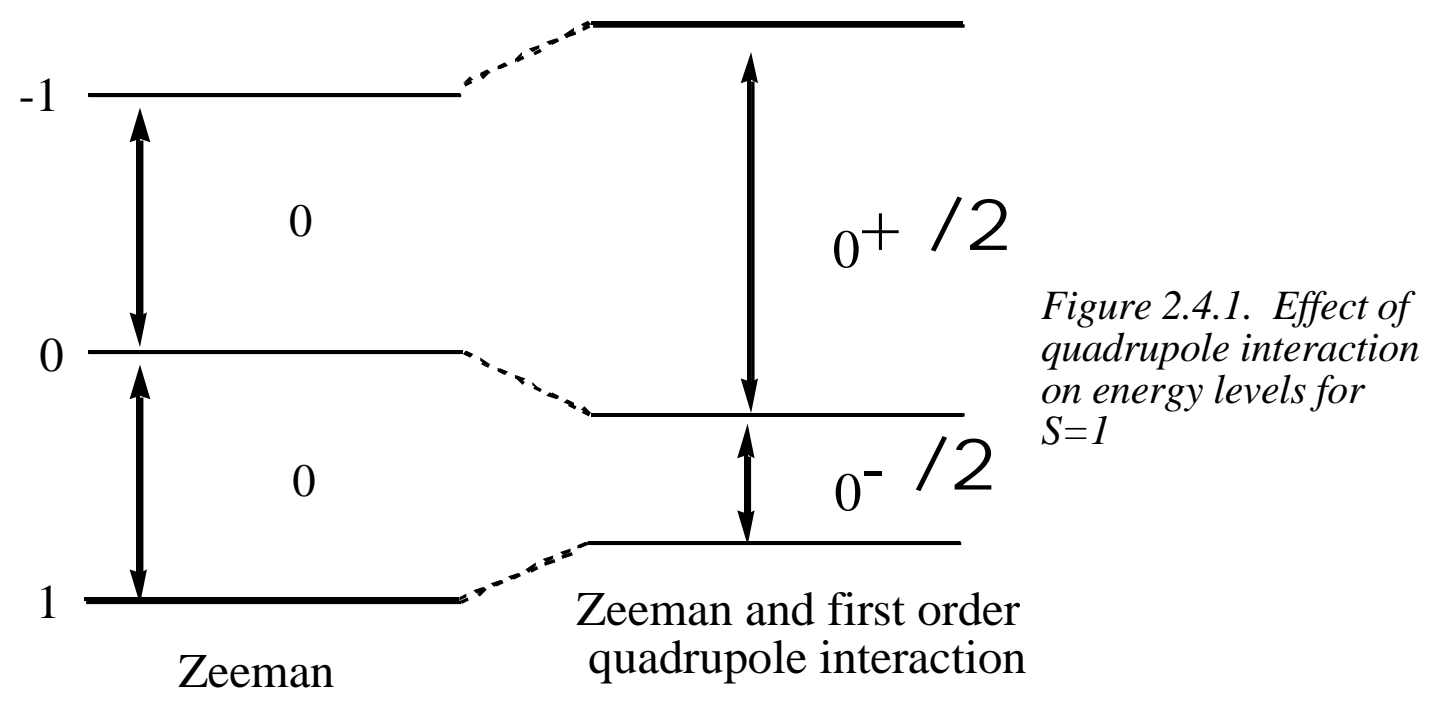

The first order quadrupole interactions cause a change in the splitting in the Zeeman levels of a spin $>1 / 2$ nucleus. For $S=1$, the energy levels are shifted by $\pm \frac{1}{2} \Delta$, where $\Delta=\frac{3 e^{2} q Q}{4 \hbar}\left(3 \cos ^{2} \theta-1\right), \frac{e^{2} q Q}{h}$ is the quadrupole coupling constant, and $e Q$ is the quadrupole moment of the nucleus. The first order quadrupole interaction for $S=1$ is shown below for $\eta=0$. 


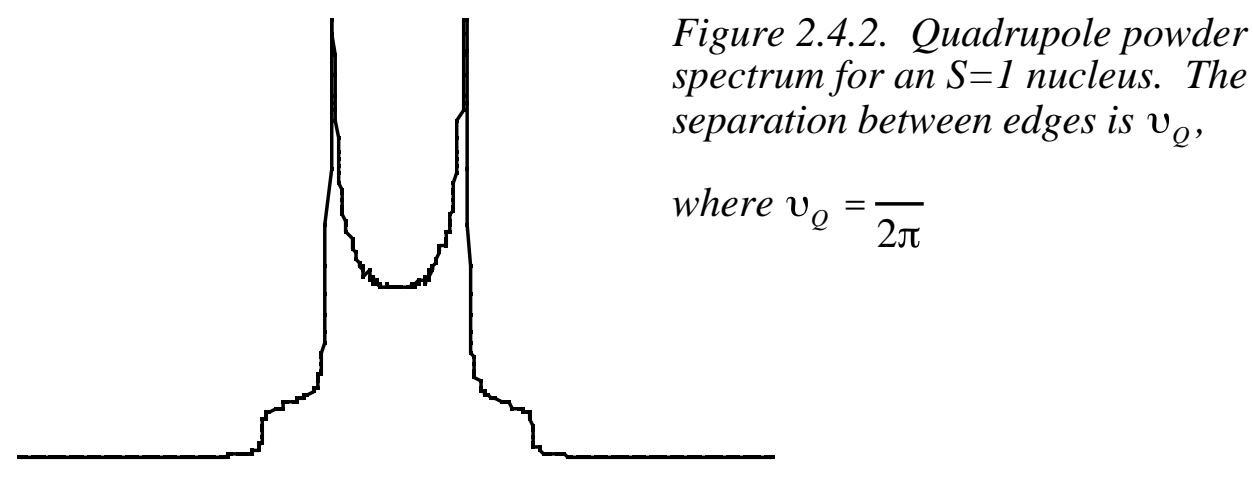

\subsection{Magic angle spinning}

In order to use solid-state NMR as a tool for determining the structure of complex organic systems, it is important to obtain high-resolution ${ }^{13} \mathrm{C}$ NMR spectra from solid

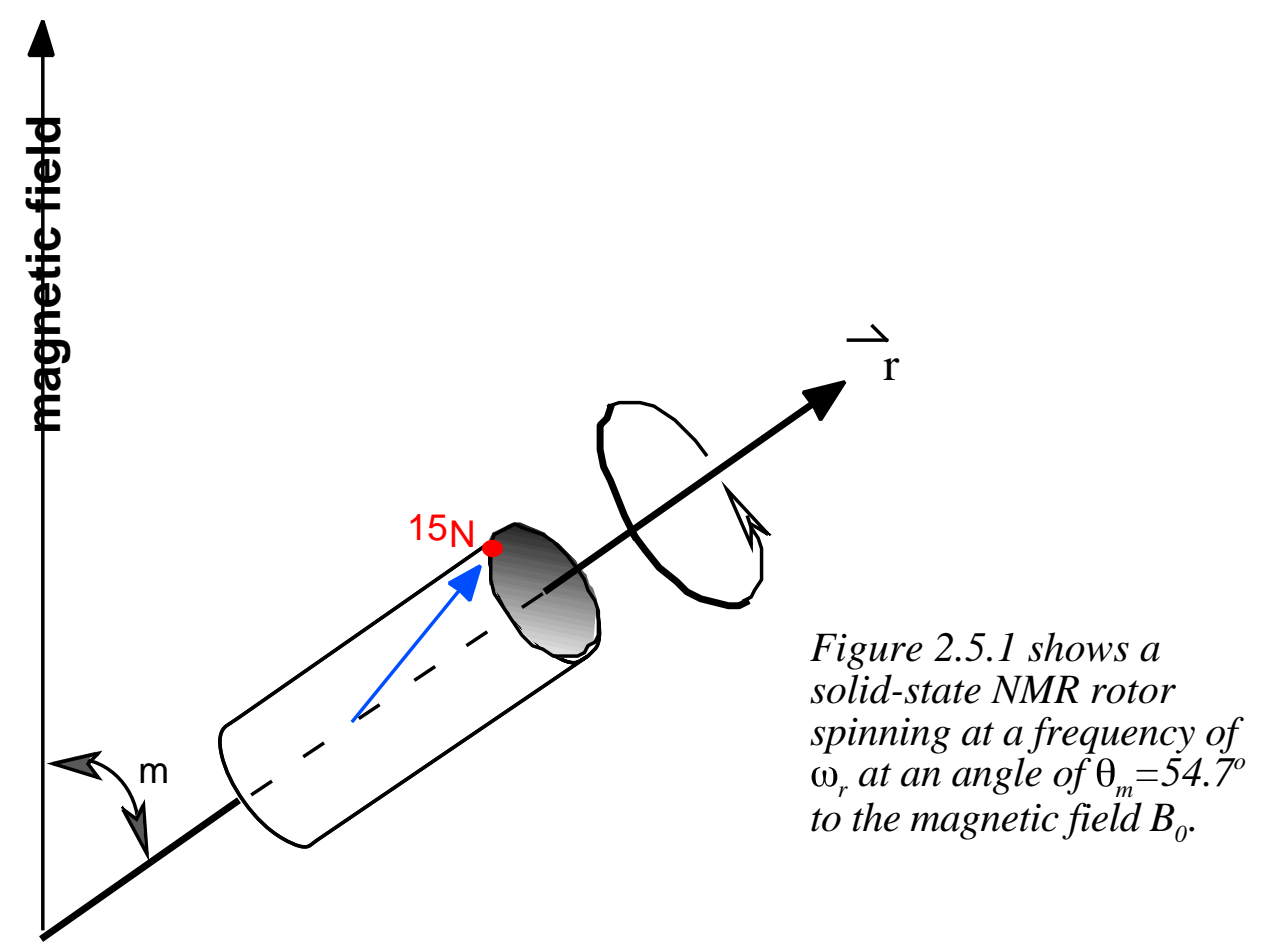


samples similar to that found in liquid NMR. In order to obtain liquid-like spectra, the averaging of the chemical shift anisotropy to the isotropic chemical shift is done by magicangle spinning (MAS). Under magic-angle spinning conditions, the internal Hamiltonians become time dependent. For the chemical shift interaction for an $\mathrm{I}=1 / 2$ nucleus such as ${ }^{13} \mathrm{C}$, the instantaneous resonance frequency becomes ${ }^{3,4}$ $\omega_{C S A}=\frac{\gamma B_{0} \sigma_{11}}{2}\left[\sin ^{2} \beta \cos 2\left(\alpha+\omega_{r} t\right)-\sqrt{2} \sin 2 \beta \cos \left(\alpha+\omega_{r} t\right)\right]+\sigma_{i s o} \gamma B_{0}$ for an axially symmetric chemical shift. As a result, mechanically spinning the NMR sample at an angle of $54.7^{\circ}$ with the respect to $\overrightarrow{B_{0}}$, as shown in Figure 2.5.1, averages the CSA to the isotropic shift. The dramatic effect of MAS is illustrated in Figure 2.5.2 for the case of ${ }^{31} \mathrm{P}$ in triphenylphosphine. At low spinning speeds, the broad static spectrum is replaced with spinning sidebands centered about the isotropic shift frequency. At very high spinning

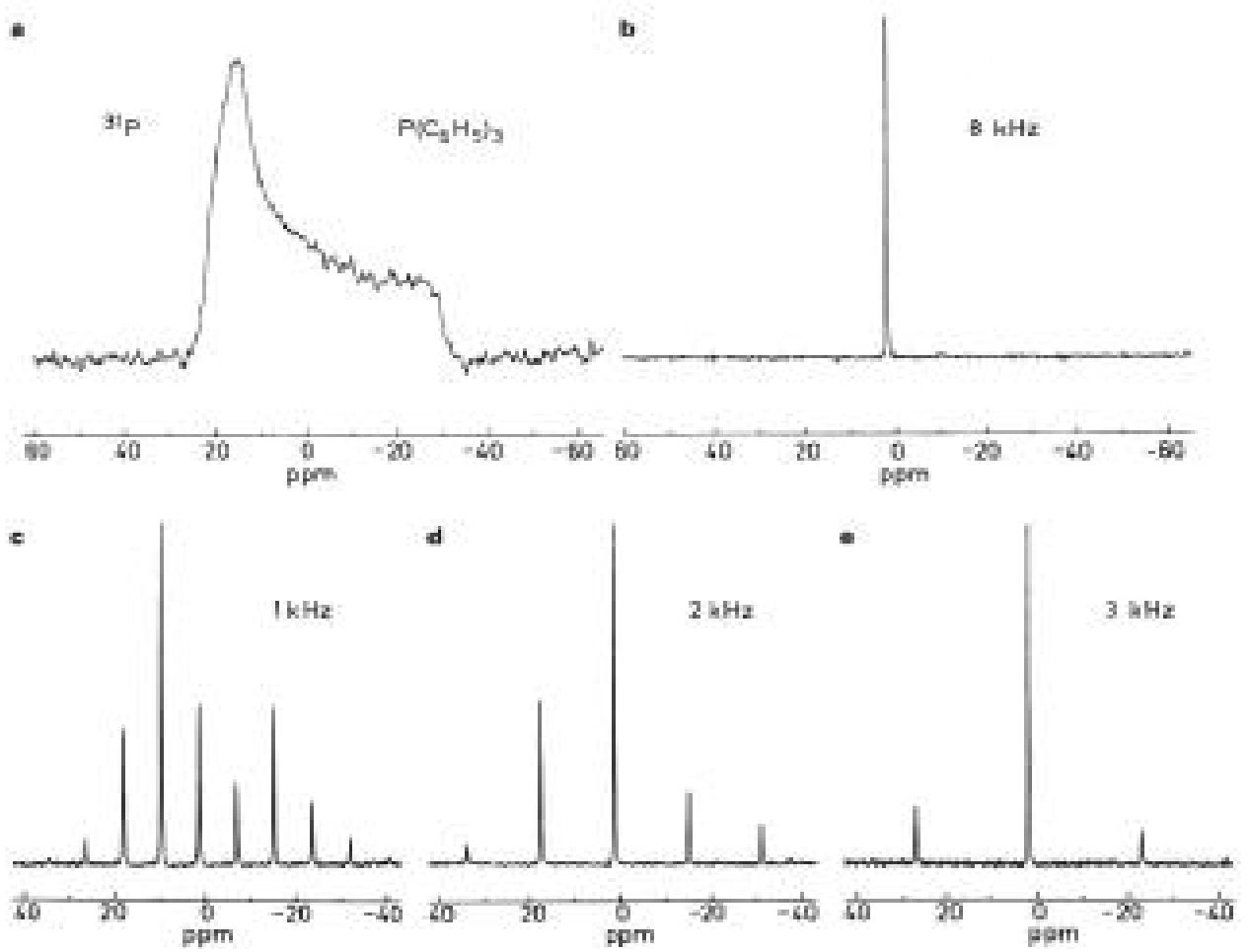

Figure 2.5.2. ${ }^{31} P$ resonance of solid triphenylphosphine without (a) and with (b) MAS. NMR frequency $121.5 \mathrm{MHz}$, (c)-(e) show spinning sidebands at different rotational frequencies. Adapted from reference 4. 
speeds, only the centerband remains and it is located at the same position found for a liquid sample.

Although MAS experiments produce sharp, liquid-like lines, they also average away useful information related to the dipole-dipole interaction. In fact, the dipole-dipole interaction is averaged to zero under MAS conditions. The ability to determine internuclear distances, both intermolecular and intramolecular is an important tool in determining molecular structure. Hence, it is necessary to recouple the dipolar interaction while maintaining high-resolution conditions.

\subsection{Rotational Echo Double Resonance NMR}

Consider a nucleus with a chemical shift anisotropy such that it has a different resonance depending on its alignment with a magnetic field. Figure 2.6.1 shows three such resonances colored such that each resonance corresponds to a molecular orientation along a particular axis. For example, when the green vector points along the z-axis (vertical axis), then the green colored resonance is observed. If the anisotropic shielding is spun uniformly

Figure 2.6.1 Three orientation dependent resonances and the orientations responsible for each. Each colored resonance occurs when the corresponding axis is aligned along the z-axis (assumed vertical).
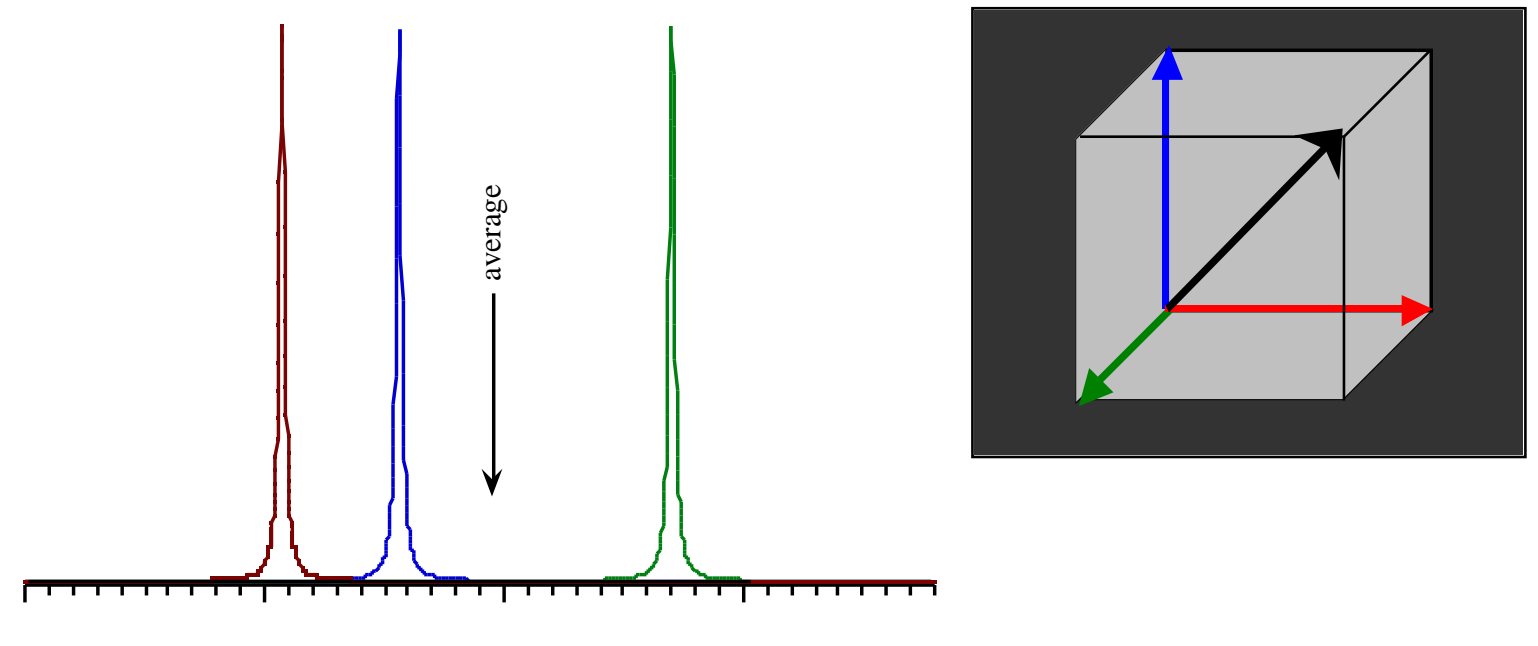
about the axis formed by the black arrow, then the anisotropy will average to the average value of all resonances since each of the three colored vectors spends the same amount of time along the z-axis.

While MAS averages the CSA to its isotropic shift value and the dipole-dipole interaction to zero, the dipole-dipole interaction can still be recovered under MAS conditions even in the presence of large chemical shift anisotropy. Figure 2.6.2 shows the effect of the orientation of an S-spin on the local field experienced by a nearby I spin. For example, when the S-spin is up, then the I-spin experiences a local field pointing downward. An NMR $\pi$ pulse applied to the S-spin produces the down state. Consequently, the local field experienced by the I-Spin now points up. In effect, S-spin $\pi$ pulses can be used to toggle the local dipole field at the I-spin in an up-down fashion and produce an average of zero., 5

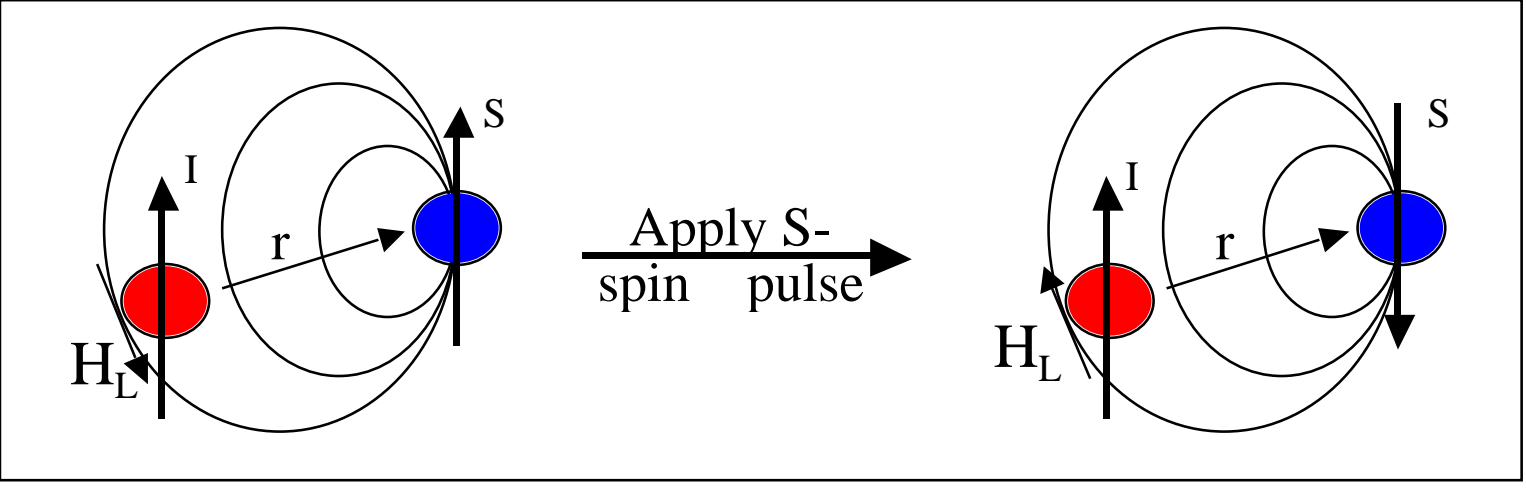

Figure 2.6.2 Manipulation of a nearby $S$-spin affects the $H_{L}$ experienced by an Ispin. The elliptical lines represent some dipole generated field lines of the S-spin.

REDOR and REAPDOR experiments allow manipulation of local dipole-generated fields to influence the echo signal formed by an I-spin nucleus. Figure 2.6 .3 shows a rotational echo, double resonance (REDOR) experiment, which consists of two separately performed pulse sequences. 

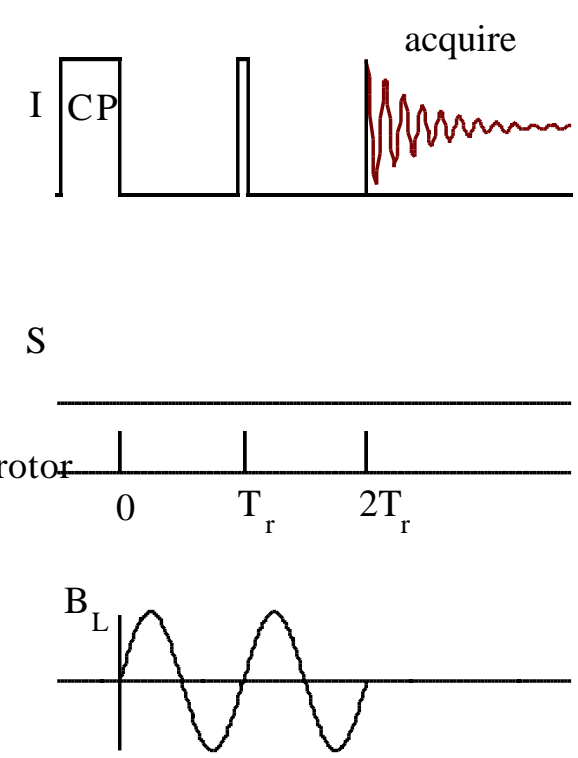
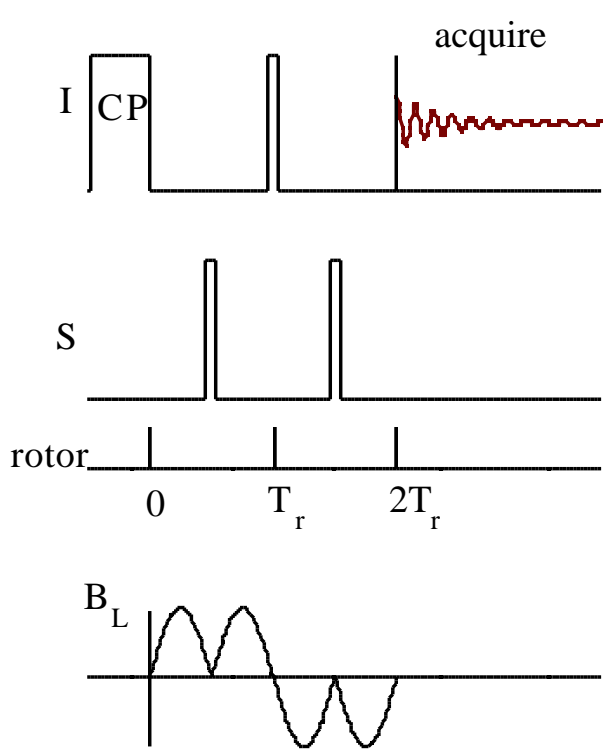

Figure 2.6.3. The REDOR experiment and corresponding local dipolar fields and signals generated. The rotor period is $T_{r}$.

A control experiment is obtained by omitting the S-spin pulses. The initial CP pulse places the magnetization of the I nucleus along the $\mathrm{x}$ axis (as shown in Figure 2.1.1). In the rotating frame, the magnetic moment precesses about $B_{L}$ for a time $\mathrm{T}_{\mathrm{r}}$, at which point an Ispin $\pi$ pulse is applied. If the initial I-spin magnetization was along the $\mathrm{x}$-axis, then a phase angle 0 is accumulated during the precession time $T_{r}$ since the dipole-dipole interaction has zero average over that time. There is no net phase accumulation during the time $\mathrm{T}_{\mathrm{r}}$ to $2 \mathrm{~T}_{\mathrm{r}}$ for the same reason. The sequence is run again with $\pi$ pulses applied to the $S$ channel as well. The $\pi$ pulses on the S channel will invert the local field experienced by the I nucleus. The local field determines the direction in which the magnetic moment precesses, inverting the local field midway through the evolution time $\mathrm{T}_{\mathrm{r}}$ prevents the I nucleus magnetization from refocusing along the $\mathrm{x}$-axis (changed the sense of precession), because the dipole-dipole interaction is no longer averaged to zero and the magnitude of the I-spin magnetization that is measured will be reduced. The reduction of the magnitude of the magnetization is known as dipolar dephasing. The amount of dephasing is dependent on the proximity of S-spin to 
the I-spin, and a measure of the amount of dephasing allows the distance between the nuclei to be determined with the REDOR experiment. Appendix A provides further details on the REDOR experiment. Theoretical plots for various spin systems have been generated with the dephased signal $\left(\mathrm{S}_{\mathrm{d}}\right)$ divided by the full signal $\left(\mathrm{S}_{0}\right)$ on the vertical axis and dipolar coupling multiplied by the evolution time on the horizontal axis. The experimental data is fitted to the theoretical curve by varying the dipolar coupling until the experimental curve fits the theoretical curve. An example of the theoretical dipolar dephasing curve is shown in Figure 2.6.4. The dipolar coupling is $D=\frac{\mu_{0} \gamma_{I} \gamma_{S} \hbar}{4 \pi r^{3}}$, and the internuclear distance can be calculated for any given $D, \gamma_{I}$, and $\gamma_{s}$. For REAPDOR, the pulse sequence is modified to contain a single dipolar dephasing pulse in the S-channel. This S-channel adiabatic passage pulse is applied for a duration of $1 / 3 \mathrm{~T}_{\mathrm{r}}$ to optimize the dipolar dephasing.

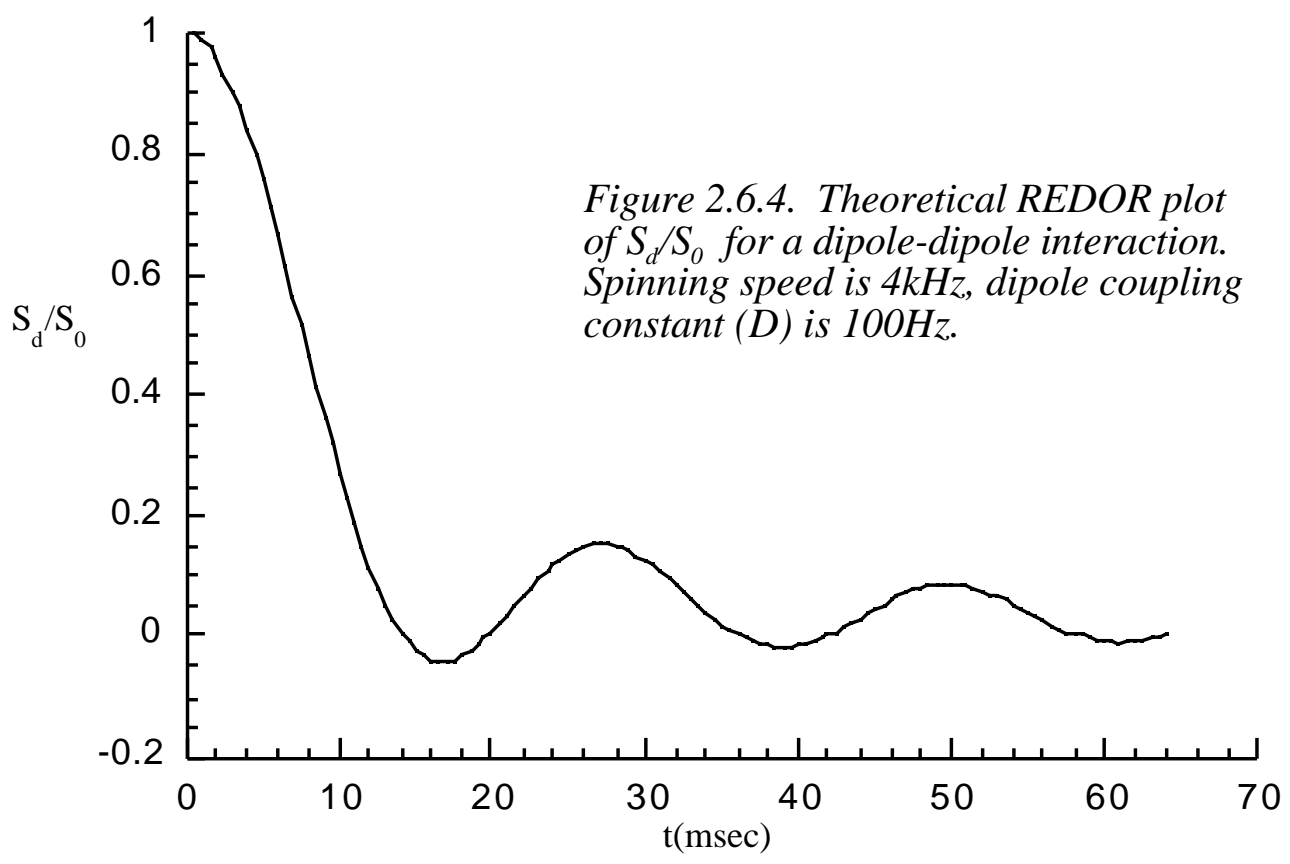




\section{Chapter 3. Experimental Methods}

The synthesis and preparation of samples is described in this chapter. The critical step in preparing TTA was the discovery of two specific zeolites whose chemical properties were first reported by Smith et al. ${ }^{8,9}$ The first zeolite is a NaY that allows selective para bromination of toluene. Because of the cost associated with isotopically labeled compounds, it is crucial that the syntheses be carried out in as few steps as possible, with as little handling as possible. Normal bromination procedures for toluene simply require the addition of bromine in the absence of light. The result is a 60:40 ortho:para mixture of bromotoluene, which then must be distilled to separate the ortho from the para. The presence of $\mathrm{NaY}$ zeolite allows for a $>99: 1$ ratio of para:ortho bromotoluene. The benefit is twofold in that 1) little to no starting material is lost on the presence of undesired product and 2) the purification step consists simply of filtering off the zeolite and removing the solvent with a rotary evaporator.

The second important zeolite $\left(\mathrm{H}^{+} \beta\right)$ is used in the synthesis of nitrotoluene. Again, the benefit of this zeolite is twofold. Normal nitration procedures for toluene require that the nitric acid being used is concentrated (70wt $\%)$. The highest concentration of ${ }^{15} \mathrm{~N}$ labeled nitric acid that is available commercially is $40 \mathrm{wt} \%$. In the presence of concentrated nitric acid, toluene reacts to form nitrotoluene in a 60:40 ratio of ortho:para. In the presence of the $\mathrm{H}^{+} \beta$ zeolite, toluene reacts with $40 \mathrm{wt} \%$ nitric acid to form a mixture of 75:25 para:ortho nitrotoluene.

\subsection{Isotope Labeling}

Isotope labeling is an important tool in NMR. ${ }^{13} \mathrm{C}$-observe REDOR and REAPDOR both involve cross polarization of the protons and carbons. If the protons on a molecule are uniformly replaced with deuterons, then cross polarization will not take place and ${ }^{13} \mathrm{C}$ resonances from that molecule can be removed from the ${ }^{13} \mathrm{C}$ spectrum. Another way that isotope labeling can be useful is to label a particular atom that has a low natural 
abundance. Labeling a carbon site with ${ }^{13} \mathrm{C}$ can enhance the signal from $1 \%$ natural abundance to $100 \%$ labeled abundance (ie, signal enhancement of 100 can be obtained). Finally, specific isotope labeling allows specific internuclear distances to be measured using REDOR and REAPDOR by enhancing the signal from a previously unlabeled ${ }^{13} \mathrm{C}$ resonance. While isotope labeling can be a very important tool for NMR, it can also be cost prohibitive. Simple molecules such as toluene or nitric acid are available with particular sites deuterated, ${ }^{15} \mathrm{~N}$ labeled, or ${ }^{13} \mathrm{C}$ labeled, but custom syntheses of more complex molecules such as TTA or PC can cost any where from hundreds of dollars per gram for deuterium labeling to tens of thousands of dollars for ${ }^{13} \mathrm{C}$ labeling per gram, per sample. In order to study the TTA/PC system with NMR, it has been necessary to synthesize the isotopically labeled samples in-house.

\subsubsection{TTA Synthesis}

Various sites in the TTA have been labelled with either ${ }^{13} \mathrm{C},{ }^{15} \mathrm{~N}$, or ${ }^{2} \mathrm{H}$. For the majority of the syntheses, commercially available ${ }^{13} \mathrm{C}$ or ${ }^{2} \mathrm{H}$ labelled toluene was used as a starting material, and TTA with the desired sites labelled was synthesized according to the scheme shown in Figure 3.1.1. ${ }^{10}$ In the case of ${ }^{15} \mathrm{~N}$ labeling, the nitration proceeds with $40 \% \mathrm{wt} / \mathrm{wt}$

${ }^{15} \mathrm{~N} \mathrm{HNO}_{3}$, which is the most concentrated product available commercially with the ${ }^{15} \mathrm{~N}$ label. In the case where the nitrogen is not labeled, concentrated $\mathrm{HNO}_{3}$ is used.

$\mathrm{NH} 4^{+} \beta$ zeolite was obtained through Zeolyst International (P.O. Box 830 Valley Forge, PA 19482; Telephone: 610-651-4621) and converted to the $\mathrm{H}^{+}$form by heating to $600^{\circ} \mathrm{C}$ overnight, refluxing twice in $1 \mathrm{M}$ aqueous ammonium acetate $(10 \mathrm{~mL} / \mathrm{g}$ zeolite), and reheating to $600^{\circ} \mathrm{C}$ overnight. It was reheated to $400^{\circ} \mathrm{C}$ for two hours immediately prior to use. $\mathrm{NaY}$ zeolite was also obtained from Zeolyst International and dried at $100^{\circ} \mathrm{C}$ under vacuum for twelve hours prior to use. All other reagents were obtained from commercial sources and used as received. The reactions were carried out in oven-dried glassware, and solvents were removed from reaction mixtures and products on a rotary evaporator at water 
aspirator pressure. Column chromatography was performed on silica gel (200-400 mesh, Natland International Corporation, P.O. Box 13391 Research Triangle Park, NC 27709). Melting points were determined using a Mettler Toledo DSC30 differential scanning calorimeter.<smiles>Cc1ccc(N)cc1</smiles>

Figure 3.1.1. Synthesis

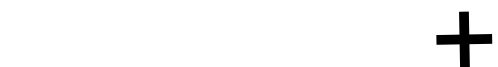
of tri-p-tolylamine<smiles>Cc1ccc(Br)cc1</smiles>

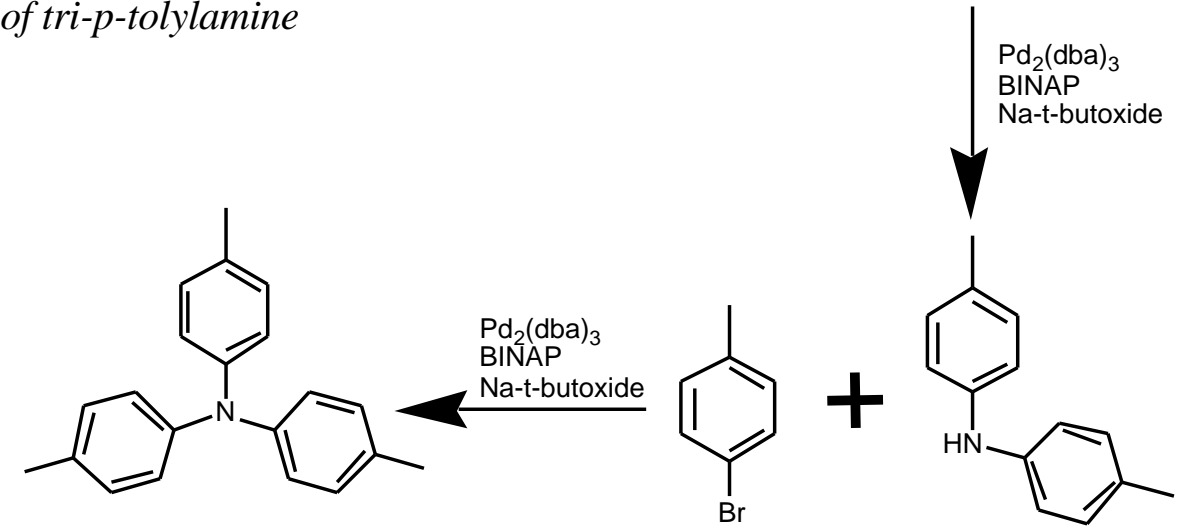

$\left({ }^{2} \mathbf{D}_{7}\right)$-4-Bromotoluene (1). A solution of bromine $(0.65 \mathrm{~mL}, 12.6 \mathrm{mmol})$ in dichloromethane $(4 \mathrm{~mL})$ was added dropwise with stirring to an aluminum foil wrapped flask containing $\mathrm{NaY}$ zeolite $(6 \mathrm{~g})$, dichloromethane $(45 \mathrm{~mL})$, and $\left(2 \mathrm{D}_{8}\right)$-toluene $(1.0 \mathrm{~g}, 9.98$ mmol). The mixture was allowed to stir at ambient temperature for $6 \mathrm{~h}$. The zeolite was removed by filtration, and the filtrate was treated with sodium hydrogen bisulfite $(10 \mathrm{~mL}$, sat. aqueous) to destroy the excess bromine. The organic phase was dried $\left(\mathrm{MgSO}_{4}\right)$, 
filtered, and the solvent was removed at reduced pressure (40 C, 2 h) affording 1 (1.44 g, $8.10 \mathrm{mmol}, 81 \%)$ as colorless crystals. $\mathrm{mp} 16{ }^{\circ} \mathrm{C} ; 13 \mathrm{C} \mathrm{NMR} \delta 136.3,130.7(\mathrm{t}, \mathrm{J}=25.2$ Hz), 130.3 (t, J = 26.8 Hz), 118.7, 19.9 (apparent septet, $\mathrm{J}=18.5 \mathrm{~Hz}$ ).

$\left({ }^{13} \mathbf{C H}_{3}\right)$-4-Bromotoluene (2). Bromine $(0.31 \mathrm{~mL}, 6.01 \mathrm{mmol})$ in dichloromethane $(4 \mathrm{~mL})$ was reacted $(6 \mathrm{~h})$ with $\mathrm{NaY}$ zeolite $(4 \mathrm{~g})$ and $\left({ }^{13} \mathrm{CH}_{3}\right)$-toluene $(500 \mathrm{mg}, 5.37 \mathrm{mmol})$ in dichloromethane $(45 \mathrm{~mL})$ and purified as described above, affording 2 (687 mg, $3.99 \mathrm{mmol}$, $74 \%$ ) as colorless crystals. mp $16{ }^{\circ} \mathrm{C} ; 1 \mathrm{H}$ NMR $\delta 7.36(\mathrm{~d}, \mathrm{~J}=8.3 \mathrm{~Hz}, 2 \mathrm{H}), 7.05(\mathrm{dd}, \mathrm{J}=$ 8.1 and 4.3 Hz, 2H), $2.29(\mathrm{~d}, \mathrm{~J}=126.8 \mathrm{~Hz}, 3 \mathrm{H}) ; 13 \mathrm{C}$ NMR $\delta 136.7(\mathrm{~d}, \mathrm{~J}=44.2 \mathrm{~Hz}), 131.3$, $130.9(\mathrm{~d}, \mathrm{~J}=3.1 \mathrm{~Hz}), 119.1,21.0$.

( $\left.\mathbf{C}^{2} \mathbf{D}_{3}\right)$-4-Bromotoluene (3). Bromine (0.31 mL, $\left.6.01 \mathrm{mmol}\right)$ in dichloromethane (4 mL) was reacted $(6 \mathrm{~h})$ with $\mathrm{NaY}$ zeolite $(4 \mathrm{~g})$ and $\left(\mathrm{C}^{2} \mathrm{D}_{3}\right)$-toluene $(500 \mathrm{mg}, 5.25 \mathrm{mmol})$ in dichloromethane (45 mL), and purified as described above, affording 3 (679 mg, $3.88 \mathrm{mmol}$, $74 \%)$ as colorless crystals. mp $16^{\circ} \mathrm{C}$; $1 \mathrm{H}$ NMR $\delta 7.36(\mathrm{~d}, \mathrm{~J}=8.3 \mathrm{~Hz}, 2 \mathrm{H}), 7.03(\mathrm{~d}, \mathrm{~J}=8.3$ Hz, 2H); 13C NMR $\delta$ 136.6, 131.2, 130.8, 119.0, 20.0 (apparent quintet, J = 24.7 Hz).

(15N)-4-Nitrotoluene (5). $\mathrm{H}^{+} \beta$ zeolite $(1.00 \mathrm{~g})$ and $\mathrm{H}^{15} \mathrm{NO}_{3}(1.0 \mathrm{~g}, 15.6 \mathrm{mmol}, 40 \%$ $\mathrm{H}^{15} \mathrm{NO}_{3}$ by weight) was added to an oven-dried round bottom flask equipped with a stir bar. The mixture was cooled (ice bath), and acetic anhydride (13 mL, $137 \mathrm{mmol})$ was added dropwise. After stirring for 30 minutes, toluene $(1.90 \mathrm{~mL}, 17.8 \mathrm{mmol})$ was added dropwise via pipette. The resulting reaction mixture was stirred at $0-5 \mathrm{C}$ for 30 minutes, and at ambient temperatre for $1.5 \mathrm{~h}$. The zeolite was removed by filtration and washed with chloroform. The filtrate was placed in a separatory funnel, and washed with water $(10 \mathrm{~mL})$. The organic phase was dried $\left(\mathrm{MgSO}_{4}\right)$ and the solvent was removed at reduced pressure. The crude product was purified by chromatography (hexanes:chloroform, 3:2) to give a mixture of $\left({ }^{15} \mathrm{~N}\right)$-4-nitrotoluene $(5)$ and $\left({ }^{15} \mathrm{~N}\right)$-2-nitrotoluene. Recrystallization from 
ethanol-water gave $\left({ }^{15} \mathrm{~N}\right)$-4-nitrotoluene $(5)(1.11 \mathrm{~g}, 8.04 \mathrm{mmol}, 52 \%)$ as faint yellow crystals. $\operatorname{mp} 48^{\circ} \mathrm{C} ; 1 \mathrm{H}$ NMR $\delta 8.10(\mathrm{dd}, \mathrm{J}=8.7$ and $2.0 \mathrm{~Hz}, 2 \mathrm{H}), 7.30(\mathrm{~d}, \mathrm{~J}=8.3 \mathrm{~Hz}, 2 \mathrm{H})$, $2.45(\mathrm{~s}, 3 \mathrm{H}) ; 13 \mathrm{C}$ NMR $\delta$ 146.3, 145.9, $129.3(\mathrm{~d}, \mathrm{~J}=2.1 \mathrm{~Hz}), 123.4,21.5$.

$\left({ }^{15} \mathbf{N},{ }^{2} \mathbf{D}_{7}\right)$-4-nitrotoluene (6). A slurry of $\mathrm{H}^{+} \beta$ zeolite (1.00 g), $\mathrm{H}^{15} \mathrm{NO}_{3}(1.0 \mathrm{~g}, 15.6$ mmol), acetic anhydride $(13 \mathrm{~mL}, 137 \mathrm{mmol})$, and $\left({ }^{2} \mathrm{D}_{8}\right)$-toluene $(2.00 \mathrm{~g}, 21.7 \mathrm{mmol})$ was reacted $(2 \mathrm{~h})$ as described above. Extraction and purification yielded 6 (1.154 g, $7.95 \mathrm{mmol}$, $51 \%$ ) as faint yellow crystals. mp $48{ }^{\circ} \mathrm{C} ; 13 \mathrm{C}$ NMR $\delta 145.9(\mathrm{~d}, \mathrm{~J}=15.4 \mathrm{~Hz}), 145.6,129.3$ (t, J = 24.2 Hz, 123.0 (t, J = 25.2 Hz), 20.6 (apparent quintet, J = 19.6 Hz).

$\left({ }^{15} \mathrm{~N}\right)$-4-Aminotoluene (7). To an oven-dried hydrogenation flask was added $\left({ }^{15} \mathrm{~N}\right)-4$ nitrotoluene (5) (1.105 g, $8.00 \mathrm{mmol})$, absolute ethanol (200 mL), and palladium on carbon (10\%, $400 \mathrm{mg})$. The vessel was sealed, pressurized to $3 \mathrm{~atm}$ of $\mathrm{H} 2$, and stirred $(2.5 \mathrm{~h})$. The catalyst was removed by filtration through Celite and the filtrate was evaporated to dryness at reduced pressure. The crude product was purified by chromatography (hexanes:chloroform, 1:7) to give $7(353 \mathrm{mg}, 3.26 \mathrm{mmol}, 41 \%)$ as colorless crystals. $\mathrm{mp} 42$ oC; $1 \mathrm{H}$ NMR d 6.96 (d, J = 8.1 Hz, 2H), 6.61 (dd, J = 8.3 and 2.0 Hz, 2H), 3.42 (br s , 2H), 2.24 (s, 3H); 13C NMR d 143.7 (d, J = 10.8 Hz), 129.7, 127.7, 115.2 (d, J = 2.6 Hz), 20.4 .

$\left({ }^{15} \mathbf{N}\right)$-4-Amino- $\left({ }^{2} \mathbf{D}_{7}\right)$-toluene (8). $\left({ }^{15} \mathrm{~N},{ }^{2} \mathrm{D}_{7}\right)$-4-nitrotoluene (6) (887 mg, $\left.6.11 \mathrm{mmol}\right)$, absolute ethanol (200 mL), and $\mathrm{Pd} / \mathrm{C}(10 \%, 400 \mathrm{mg})$ was reacted and purified as described above (2.5 h) affording $8(370 \mathrm{mg}, 321 \mathrm{mmol}, 53 \%)$ as colorless crystals. $\mathrm{mp} 42^{\circ} \mathrm{C} ; 1 \mathrm{H}$ NMR $\delta 3.42$ (br s); 13C NMR $\delta 143.6$ (d, J = 10.3 Hz), $129.2(\mathrm{t}, \mathrm{J}=23.2 \mathrm{~Hz}), 127.3$, $114.8(\mathrm{t}, \mathrm{J}=23.2 \mathrm{~Hz}), 19.7(\mathrm{~m}, \mathrm{~J}=18.8 \mathrm{~Hz})$. 
$\left({ }^{15} \mathrm{~N}\right)-\mathrm{N}, \mathrm{N}$-bis(4-methylphenyl)-4-methylbenzeneamine $(9)$ and $\left({ }^{15} \mathrm{~N}\right)-\mathrm{N}-(4-$ methylphenyl)-4-methylbenzeneamine (10). To an oven-dried ACE-Glass pressure tube was added 4-bromotoluene (1.28 g, 7.50 mmol), $\left({ }^{15} \mathrm{~N}\right)-4$-aminotoluene (7) $(353 \mathrm{mg}$, $3.27 \mathrm{mmol}$ ), sodium t-butoxide (432 $\mathrm{mg}, 4.49 \mathrm{mmol}$ ), tris(dibenzylideneacetone) dipalladium(0) $\left(\mathrm{Pd}_{2}(\mathrm{dba})_{3}\right)(60 \mathrm{mg}, 0.065 \mathrm{mmol}),(\mathrm{R})-(+)-2,2^{\prime}$-bis(diphenylphosphino)1,1'-binapthyl (BINAP) (122 mg, $0.196 \mathrm{mmol})$, and a small stir bar. The reaction vessel was flushed with $\mathrm{N}_{2}$ and sealed with a Teflon screwcap, then heated to $90^{\circ} \mathrm{C}$ for four days with stirring. The mixture was allowed to cool to ambient temperature, taken up in diethyl ether $(50 \mathrm{~mL})$, and filtered through Celite. Solvent removal and purification by chromatography (hexanes:chloroform, 5:1) gave, in order of elution, $9(202 \mathrm{mg}, 0.70 \mathrm{mmol}$, $21 \%$ ) and $\mathbf{1 0}(350 \mathrm{mg}, 1.77 \mathrm{mmol}, 54 \%)$ both as faint yellow crystals. Analytical data for $\mathbf{9}$ : mp $114^{\circ} \mathrm{C}$; $1 \mathrm{H}$ NMR $\delta 7.03(\mathrm{~d}, \mathrm{~J}=8.5 \mathrm{~Hz}, 6 \mathrm{H}), 6.95(\mathrm{~d}, \mathrm{~J}=8.3 \mathrm{~Hz}, 6 \mathrm{H}), 2.29(\mathrm{~s}, 9 \mathrm{H}) ; 13 \mathrm{C}$ NMR $\delta 145.7(\mathrm{~d}, \mathrm{~J}=15.4 \mathrm{~Hz}), 131.7,129.7,123.8,20.7$. Analytical data for 10: $\mathrm{mp} 78^{\circ} \mathrm{C}$; $1 \mathrm{H}$ NMR $\delta 7.05(\mathrm{~d}, \mathrm{~J}=8.3 \mathrm{~Hz}, 2 \mathrm{H}), 6.92(\mathrm{dd}, \mathrm{J}=8.3$ and 1.8, 2H), $5.50(\mathrm{br} d, \mathrm{~J}=27 \mathrm{~Hz}$, 1H), $2.28(\mathrm{~s}, 6 \mathrm{H}) ; 13 \mathrm{C}$ NMR $\delta 141.2(\mathrm{~d}, \mathrm{~J}=14.4 \mathrm{~Hz}), 130.2,129.9,118.0,20.7$.

$\left({ }^{15} \mathrm{~N}\right) \mathrm{N}, \mathrm{N}$-bis(4-methylphenyl)-4-methylbenzeneamine (9). Reaction of 4bromotoluene (342 mg, $2.00 \mathrm{mmol}),(15 \mathrm{~N})-\mathrm{N}$-(4-methylphenyl)-4-methylbenzeneamine (10) (349 mg, $1.77 \mathrm{mmol}$ ), sodium t-butoxide (480 mg, $5.00 \mathrm{mmol}$ ), $\mathrm{Pd}_{2}(\mathrm{dba})_{3}(32.4 \mathrm{mg}$, $0.035 \mathrm{mmol})$, and BINAP (66.1 $\mathrm{mg}, 0.106 \mathrm{mmol})$, as described above $\left(90^{\circ} \mathrm{C}, 4\right.$ days $)$ gave, after chromatography (hexanes-chloroform, 6:1), $9(361 \mathrm{mg}, 1.25 \mathrm{mmol}, 71 \%)$ as faint yellow crystals.

$\left({ }^{15} \mathrm{~N},{ }^{2} \mathrm{D}_{21}\right)$-N,N-bis(4-methylphenyl)-4-methylbenzeneamine (11) and $\left({ }^{15} \mathrm{~N},{ }^{2} \mathrm{D}_{14}\right) \mathrm{N}$ (4-methylphenyl)-4-methylbenzeneamine (12). Reaction of $\left({ }^{2} \mathrm{D}_{7}\right)$-4-bromo-1-toluene (1) (641 mg, $3.60 \mathrm{mmol}),\left({ }^{15} \mathrm{~N},{ }^{2} \mathrm{D}_{7}\right)$-4-aminotoluene (8) (370 mg, $3.42 \mathrm{mmol}$ ), sodium tbutoxide (461 mg, $4.80 \mathrm{mmol}), \mathrm{Pd}_{2}(\mathrm{dba})_{3}(62.7 \mathrm{mg}, 0.068 \mathrm{mmol})$, and BINAP (128 mg, 
$0.205 \mathrm{mmol})$ as described above $\left(90^{\circ} \mathrm{C}, 4\right.$ days $)$ gave, after extraction and purification, 11 (108 mg, $0.37 \mathrm{mmol}, 11 \%)$ and $12(367 \mathrm{mg}, 1.85 \mathrm{mmol}, 54 \%)$ both as faint yellow crystals. Analytical data for 11: $\mathrm{mp} 114^{\circ} \mathrm{C} ; 13 \mathrm{C}$ NMR $\delta 145.5(\mathrm{~d}, \mathrm{~J}=16.0 \mathrm{~Hz}), 131.1,129.3(\mathrm{t}, \mathrm{J}=$ 23.7 Hz), 123.4 (t, J = 23.2 Hz), 19.9 (apparent sextet, $\mathrm{J}=18.5 \mathrm{~Hz}$ ).

Analytical data for 12: $\mathrm{mp} 78^{\circ} \mathrm{C}$; $1 \mathrm{H}$ NMR $\delta 5.55$ (br s); $13 \mathrm{C}$ NMR $\delta 140.9$ (d, J = 14.9 $\mathrm{Hz}), 129.6,129.2(\mathrm{dd}, \mathrm{J}=23.2$ and $16.0 \mathrm{~Hz}), 117.2(\mathrm{dd}, \mathrm{J}=47.3$ and 23.2), 19.7 (apparent sextet, $\mathrm{J}=19.0 \mathrm{~Hz}$ ).

$\left({ }^{15} \mathbf{N},{ }^{2} \mathbf{D}_{21}\right) \mathrm{N}, \mathbf{N}$-bis(4-methylphenyl)-4-methylbenzeneamine (11). Reaction of $\left({ }^{2} \mathrm{D}_{7}\right)$-4-bromotoluene (8) (391 mg, $\left.2.18 \mathrm{mmol}\right),\left({ }^{15} \mathrm{~N},{ }^{2} \mathrm{D}_{14}\right) \mathrm{N}-(4-m e t h y l p h e n y l)-4-$ methylbenzenamine (12) (367 mg, $1.79 \mathrm{mmol}$ ), sodium t-butoxide (506 mg, $5.26 \mathrm{mmol}$ ), $\mathrm{Pd}_{2}(\mathrm{dba})_{3}(34 \mathrm{mg}, 0.037 \mathrm{mmol})$, and BINAP $(69.6 \mathrm{mg}, 0.112 \mathrm{mmol})$ as described above (90 ${ }^{\circ} \mathrm{C}, 4$ days) gave, after extractionand purification, 11 (335 mg, $\left.1.08 \mathrm{mmol}, 60 \%\right)$ as faint yellow crystals.

N,N-bis(4-methylphenyl)-4-(13CH3)-methylbenzeneamine (13). Reaction of $\left({ }^{13} \mathrm{CH}_{3}\right.$ )-4-bromotoluene (3) (342 mg, $\left.1.99 \mathrm{mmol}\right)$, N-(4-methylphenyl)-4methylbenzenamine (473 mg, $2.40 \mathrm{mmol}$ ), sodium t-butoxide (269 mg, $2.80 \mathrm{mmol}$ ), $\operatorname{Pd}_{2}(\mathrm{dba})_{3}(18.4 \mathrm{mg}, 0.037 \mathrm{mmol})$, and BINAP $(37.4 \mathrm{mg}, 0.11 \mathrm{mmol})$ as described above $\left(90^{\circ} \mathrm{C}, 4\right.$ days) gave, after extraction and purification, 13 (467 mg, $\left.1.62 \mathrm{mmol}, 67 \%\right)$ as faint yellow crystals. $\mathrm{mp} 114^{\circ} \mathrm{C} ; 1 \mathrm{H}$ NMR $\delta 7.02(\mathrm{~d}, \mathrm{~J}=8.5 \mathrm{~Hz}, 6 \mathrm{H}), 6.94(\mathrm{~d}, \mathrm{~J}=8.5 \mathrm{~Hz}, 6 \mathrm{H})$, $2.29(\mathrm{~d}, \mathrm{~J}=126.2 \mathrm{~Hz}, 3 \mathrm{H}), 2.29(\mathrm{~s}, 6 \mathrm{H}) ; 13 \mathrm{C}$ NMR $\delta$ 145.7, 131.7, 129.7, 123.8, 20.8.

\subsubsection{Sample Preparation:}

Films were prepared by dissolving appropriate weight:weight ratios of TTA and polycarbonate in methylene chloride. The TTA/PC:methylene chloride ratio is kept at $1 \%$ wt:vol. This solution is sealed in a flask to prevent solvent evaporation and stirred overnight 
before being caste in $20 \mathrm{~mL}$ aliquots onto flat glass plates $\left(2100 \mathrm{~cm}^{2}\right)$ and spread with a glass roller. The plates are allowed to dry $5 \mathrm{~h}$ at room temperature then are scraped from the glass and dried under vacuum at $360 \mathrm{~K}$ to remove any remaining solvent.

\subsection{NMR}

REDOR and REAPDOR solid state NMR measurements were made on a homebuilt triple-channel spectrometer ${ }^{11}$ operating at a proton frequency of $151.394 \mathrm{MHz}$ and employing a Tecmag Libra pulse programmer. The triple tuned MAS probe uses a Chemagnetics $7.5 \mathrm{~mm}$ spinning assembly and is of a transmission-line design. Typical proton rf field strengths were $50 \mathrm{kHz}$ for $\mathrm{CP}$ and $110 \mathrm{kHz}$ for proton decoupling. All $\mathrm{CP}$ contact times were $1 \mathrm{~ms},{ }^{13} \mathrm{C},{ }^{14} \mathrm{~N}$, and ${ }^{2} \mathrm{H}$ rf field strengths were 50,44 , and $45 \mathrm{kHz}$ respectively. Sample spinning rates were $1996 \mathrm{~Hz}$ for the ${ }^{13} \mathrm{C}-{ }^{14} \mathrm{~N}$ REAPDOR experiments and $3125 \mathrm{~Hz}$ for the ${ }^{13} \mathrm{C}-{ }^{2} \mathrm{D}$ REDOR experiments. Spinning rate stability is critical for the experiments and was controlled to within $0.2 \mathrm{~Hz}$ of the desired values. A schematic of the spectrometer is shown in Figure 3.2.1.

The Tecmag pulse programmer is basically a gating device that controls the radio frequency (rf) transmitter unit by gating rf pulses of desired phases $\left(0^{\circ}, 90^{\circ}, 180^{\circ}\right.$, and $\left.270^{\circ}\right)$ at certain times of the NMR experiment. The pulse programmer also gates the detector located in the home-built receiver section. In addition, filters and the signal digitizer are located in the Tecmag pulse programmer. Pulse sequences and data manipulation are performed on a Macintosh IIci computer using Tecmag software.

The rf unit is based on heterodyning at $25 \mathrm{MHz}$. Rf signals at $25 \mathrm{MHz}$ are generated for the four desired phases. The power splitters are located inside the homebuilt rf transmitter unit (shown outside for clarity). Heterodyning at $25 \mathrm{MHz}$ is used since all phase generation can be performed at a common frequency which is independent of any nuclear resonance frequency. The $25 \mathrm{MHz}$ pulse signals are mixed with the $25 \mathrm{MHz}+\mathrm{v}_{0}$ frequency generated by the synthesizer to generate the NMR frequency $v_{0}$, which is sent to 
a 25 Watt AMT amplifier (for ${ }^{1} \mathrm{H}$ ). The rf pulse is further amplified by a $2 \mathrm{~kW}$ att Creative Electronics tuned amplifier and this amplified signal is sent to the probe via the duplexer.

The NMR response signal at $v_{0}$ produced by the sample in the probe is detected (via the duplexer) by a low power Miteq high-gain preamp. This signal is then mixed with a frequency at $25 \mathrm{MHz}+\mathrm{v}_{0}$ to produce a frequency modulated signal at $25 \mathrm{MHz}$. The signal is then passed a $90^{\circ}$ splitter to generate orthogonal signals ( $\mathrm{x}$ and $\mathrm{y}$ ) components. The signal is mixed once again at $25 \mathrm{MHz}$ to produce an "audio" signal that is filtered, digitized, and stored in memory. 


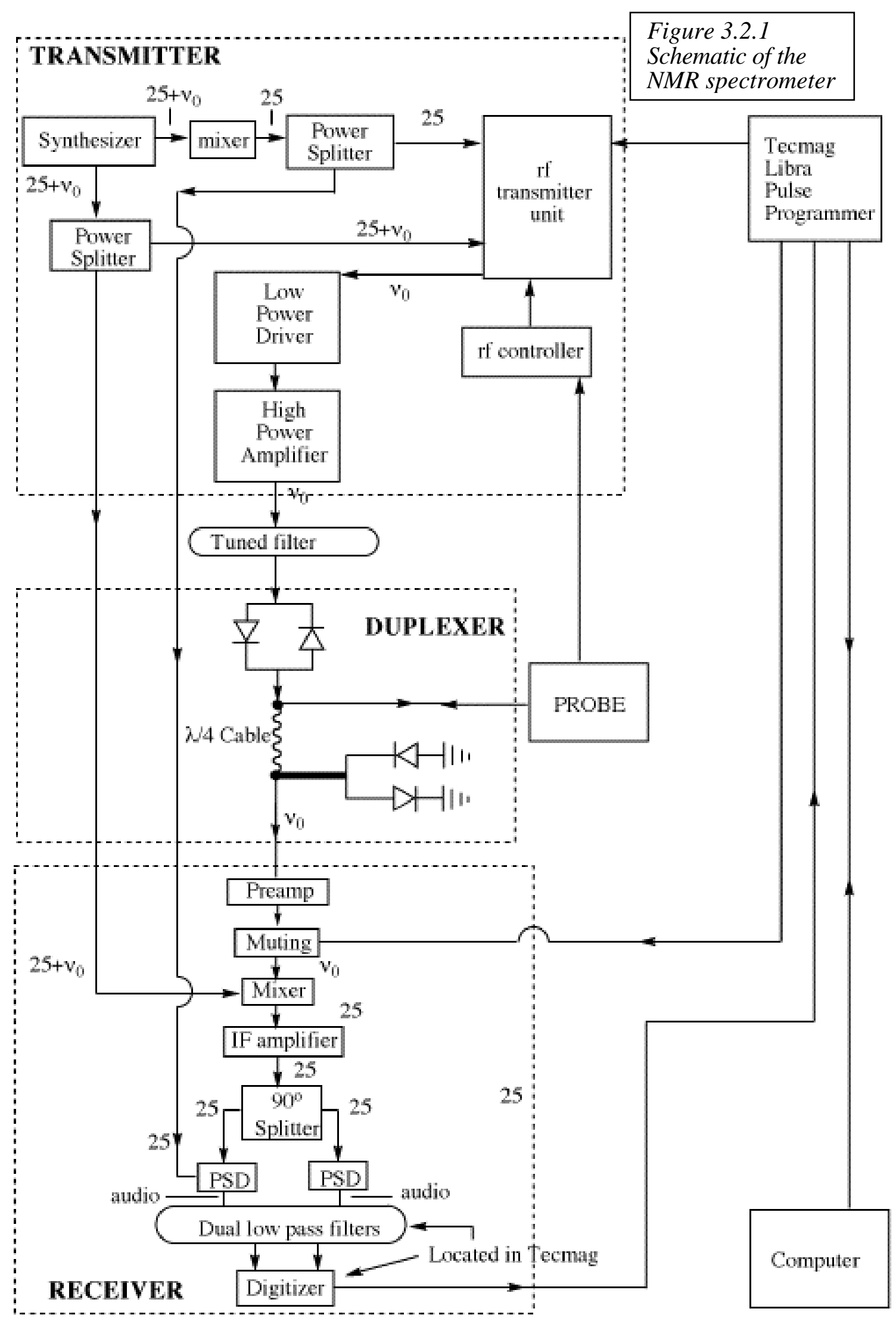




\section{Chapter 4. Experimental Results and Discussion}

A series of REDOR and REAPDOR experiments were performed to obtain structural information of amorphous TTA:PC blends. These NMR experiments are ideally suited to extract internuclear distances and work for amorphous and glassy systems.

\subsection{Evidence for Miscibility}

It is important to show that the TTA dopant molecules form a miscible blend with polycarbonate. Traditionally, the proof of miscibility for blends involves differential scanning calorimetry (DSC) and requires a change in the glass transition of the individual components, or a reduction in the melting points of the individual components. Although DSC provides a quick result, the interpretation of the data can be difficult. Miscibility can also be shown using solid-state NMR. ${ }^{13} \mathrm{C}-{ }^{2} \mathrm{H}$ REDOR data from a blend of fully deuterated TTA $\left(\mathrm{d}_{21}\right.$-TTA) and PC can show miscibility. Analysis of this data provides the extent to which the TTA and PC are blended. Figure 4.1.1 shows the structures of PC and TTA. The labeled sites identify the ${ }^{13} \mathrm{C}$ resonances that appear in the ${ }^{13} \mathrm{C}$ MAS NMR spectra shown in Figure 4.1.2. Unlabeled peaks are spinning sidebands. In the case of the

${ }^{13} \mathrm{C}$ spectrum of a TTA/PC blend (bottom spectrum of Figure 4.1.2), some ${ }^{13} \mathrm{C}$ resonances of both molecules overlap. For example, it is clear that the ${ }^{13} \mathrm{C}$ resonances for the $\mathrm{A}$ position of PC and the A position of TTA cannot be resolved. Complete deuteration of one species can simplify the ${ }^{13} \mathrm{C}$ NMR spectrum. The upper spectrum of Figure 4.1.2 is from a blend of fully deuterated TTA (30 wt $\%)$ and PC (70 wt \%). Because REDOR and REAPDOR make use of cross polarization (CP) between ${ }^{13} \mathrm{C}$ and protons, ${ }^{13} \mathrm{C}$ resonances are not observed for ${ }^{13} \mathrm{C}$ nuclei located on fully deuterated molecules, especially if the $\mathrm{CP}$ contact time is kept short. Hence, the only resonances observed in the top spectrum of Figure 4.1.2 are from the $\mathrm{PC}{ }^{13} \mathrm{C}$ nuclei. 


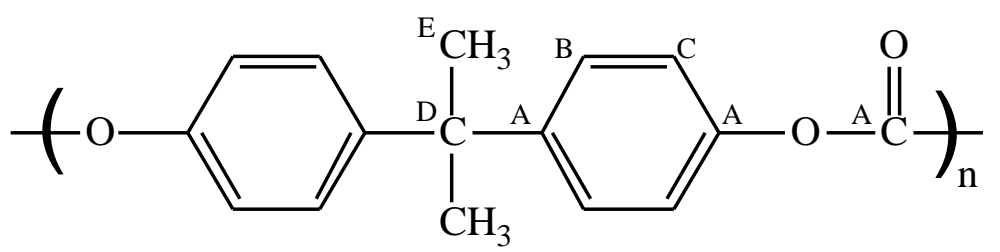

bisphenol-A-polycarbonate

Figure 4.1.1. The PC monomer and TTA molecule with ${ }^{13} \mathrm{C}$ positions<smiles>C[14c]1ccc([13CH2])c[13c]1</smiles>
from spectra in Figure 4.1.2 labeled.

tri-p-tolylamine

Comparing the ${ }^{2} \mathrm{H}$ - labeled and unlabeled blend spectra, it is evident that only the methyl ${ }^{13} \mathrm{C}$ (F in the Figures) of TTA is completely resolved from the $\mathrm{PC}{ }^{13} \mathrm{C}$ resonances, and the $\mathrm{B}$ ' resonance of TTA is partially resolved.

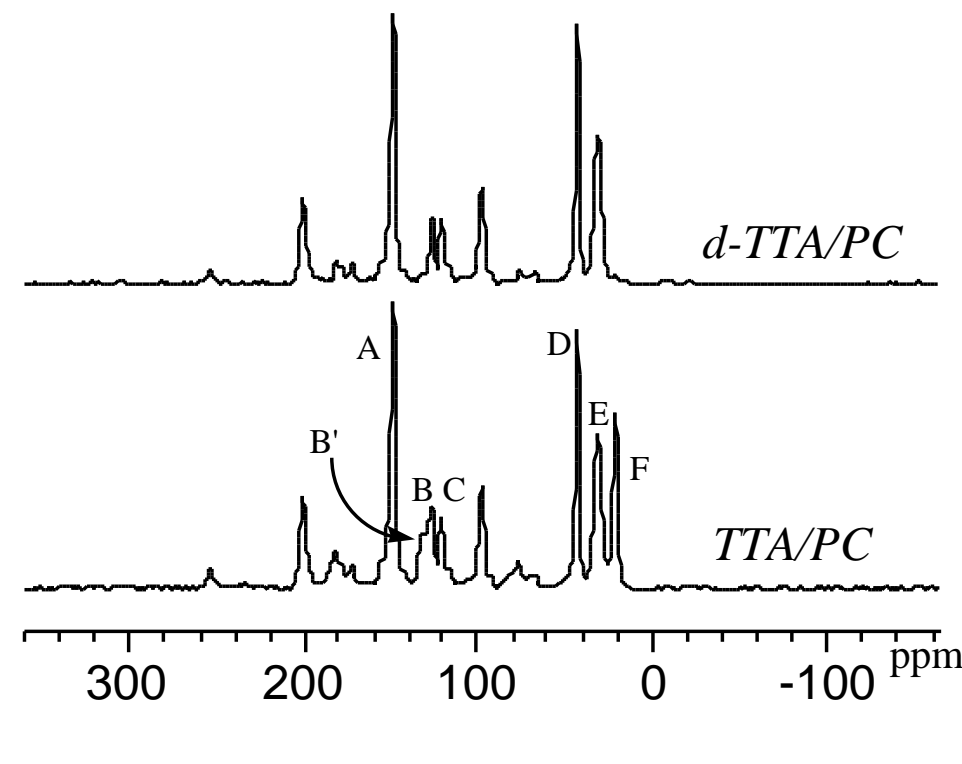

Figure 4.1.2. Spectra showing the ${ }^{13} \mathrm{C}$ peak positions for TTA and PC 
Spectral overlap can be a problem with our experiments, because the distance measurements made are dependent on the change in the signal intensity. Consider measuring a distance from the A carbon on the TTA molecule to a position on the polycarbonate or to a position on another TTA molecule. A simple REDOR experiment at

${ }^{13} \mathrm{C}$ natural abundance would yield little useful information because any changes in the peak height of the TTA's A position ${ }^{13} \mathrm{C}$ resonance would be skewed by dephasing (or lack thereof) from the A position ${ }^{13} \mathrm{C}$ resonances of the polycarbonate. If the A position of the TTA were enriched with a ${ }^{13} \mathrm{C}$, the ${ }^{13} \mathrm{C}$ signal from the A position would increase substantially as a result of the labeling and would be dominated by the contribution from TTA. Any changes in signal height from dephased atoms other than the TTA A position could be factored out (using a sample blank and comparing it to the labeled results) or considered negligible due to the very intense signal from the labeled TTA position.

The blend can be shown to be miscible by performing ${ }^{13} \mathrm{C}-{ }^{2} \mathrm{H}$ REDOR experiments on a blend made with fully deuterated TTA. The full and difference ${ }^{13} \mathrm{C}^{2} \mathrm{H}$ REDOR spectra are shown in Figure 4.1 .3 for a blend of $30 \mathrm{wt} \% \mathrm{~d}_{21}$-TTA and $70 \mathrm{wt} \%$ PC. The full spectrum shows the ${ }^{13} \mathrm{C}$ resonances of the $\mathrm{PC}$ with no dipolar dephasing from the deuterons of the TTA. Only the ${ }^{13} \mathrm{C}$ resonances from the PC are present since the TTA is fully deuterated and makes no contribution to the CPMAS spectrum. A dipolar dephased

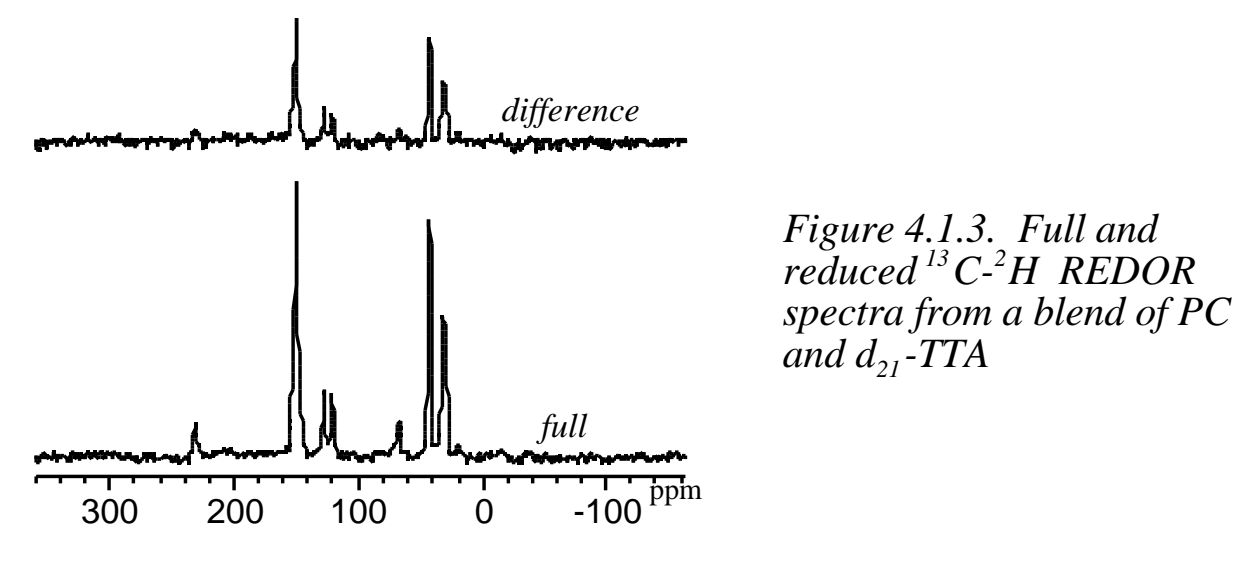


spectrum (reduced spectrum) with a dipolar evolution period of $19 \mathrm{msec}$ was acquired by applying a ${ }^{2} \mathrm{H}$ dipolar recoupling pulse. The difference spectrum, defined as the full spectrum minus the reduced spectrum, shown in Figure 4.1.3, shows that each of the PC ${ }^{13} \mathrm{C}$ resonances is dephased by the deuterons on the $\mathrm{d}_{21}$-TTA. The presence of a difference spectrum for any of the $\mathrm{PC}{ }^{13} \mathrm{C}$ resonances indicates that the ${ }^{2} \mathrm{H}$ nuclei of the $\mathrm{d}_{21}$-TTA are within 6.5 angtroms of the PC carbon atoms. In fact, all carbon sites of PC show similar

${ }^{13} \mathrm{C}$ signal loss upon application of the ${ }^{2} \mathrm{H}$ pulses which implies that there is no specific site on the PC chain to which the TTA molecules are more attracted. The combination of the proximity of the TTA atoms to the PC atoms, along with the lack of a particular site on the PC chain to which the TTA is attracted, show that the TTA forms a miscible blend with PC at this composition. A plot of $\Delta \mathrm{S} / \mathrm{S}$ for this blend is shown in Figure 4.1.4. $\Delta \mathrm{S} / \mathrm{S}$ is defined as 1 - reduced intensity/full intensity. Complete dephasing would correspond to a $\Delta \mathrm{S} / \mathrm{S}$ value of 1 . The $\Delta \mathrm{S} / \mathrm{S}$ data reaches a limiting value of 0.44 and indicates that $44 \%$ of the PC carbon atoms are in intimate contact with the deuterons of the TTA.

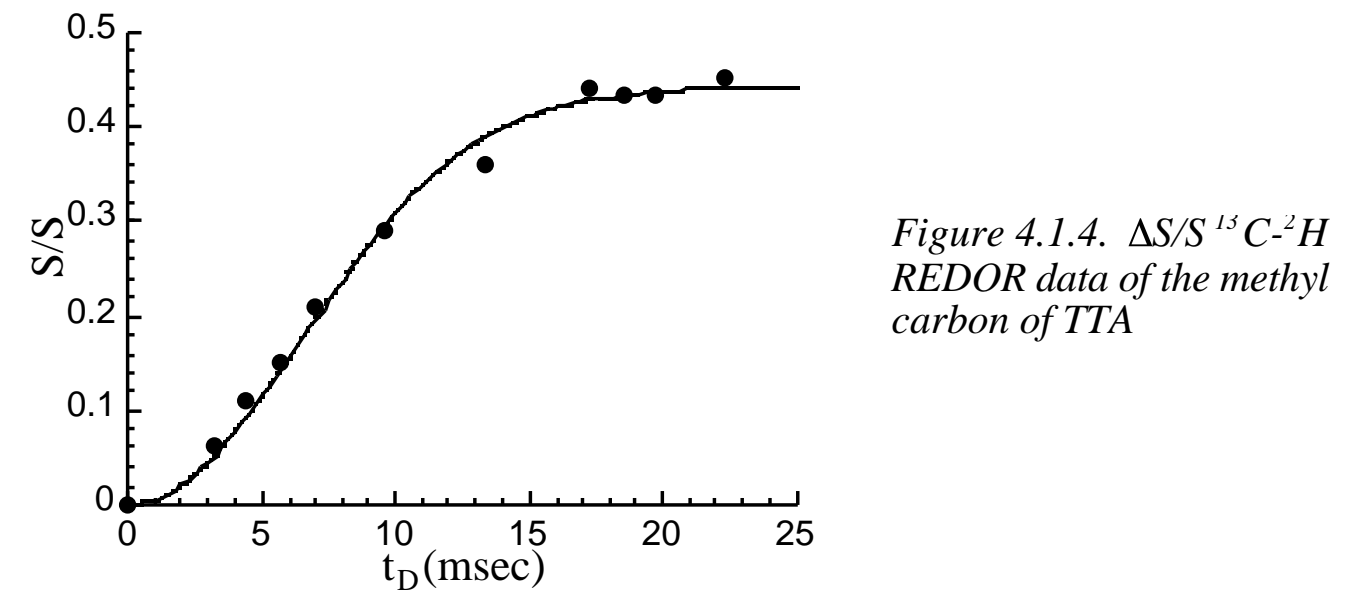

Although doped polymer systems such as the TTA/PC blend are normally considered to follow a uniform dispersion model, there has been previous work to suggest a structuring of the dopant molecules within the polymer matrix. Hewitt et. al published cross 
polarization experiments in 1984 that seemed to indicate a stacking of the TTA molecules in the PC matrix. With that in mind, ${ }^{13} \mathrm{C}-{ }^{14} \mathrm{~N}$ REAPDOR experiments on $\mathrm{d}_{21}$-TTA/PC blends were performed. The full and reduced spectra for the ${ }^{13} \mathrm{C}-{ }^{14} \mathrm{~N}$ REAPDOR experiment are presented in Figure 4.1.5 superposed together. As can be seen in the spectra, the ${ }^{13} \mathrm{C}$ resonances of the PC are hardly dephased by the $\mathrm{TTA}^{14} \mathrm{~N}$. There are three possible explanations for this lack of dephasing, one being that the $14 \mathrm{~N} \mathrm{~T}_{1}$ is very short and another that the ${ }^{14} \mathrm{~N}$ quadrupole coupling constant is too large for this experiment to work. A third possibility is that the ${ }^{14} \mathrm{~N}$ is too far away from the PC carbons for dephasing to occur. The REAPDOR experiment is able to measure dipolar dephasing to a distance of approximately $6.5 \stackrel{\circ}{\mathrm{A}}$. If we consider the previous data showing that the TTA deuterons are in close contact

a)

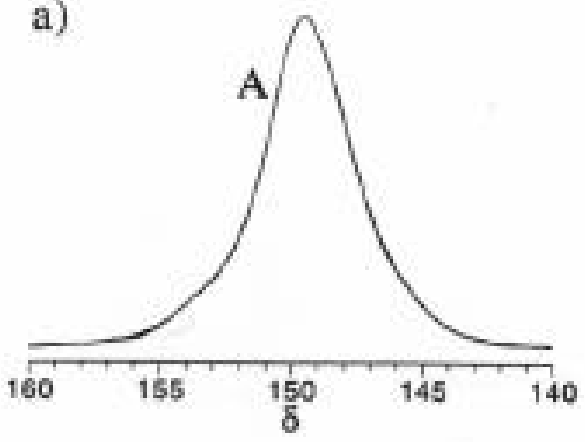

c)

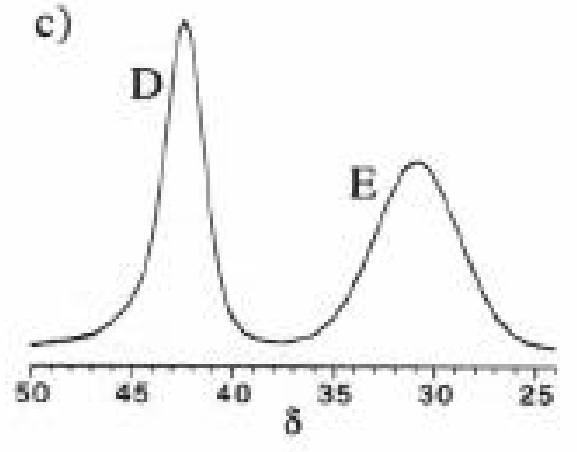

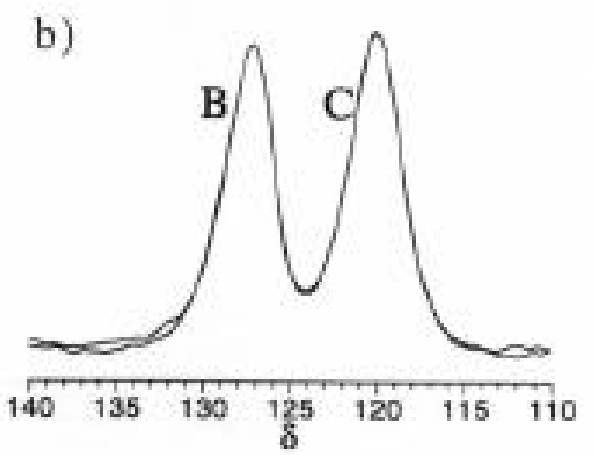

d)

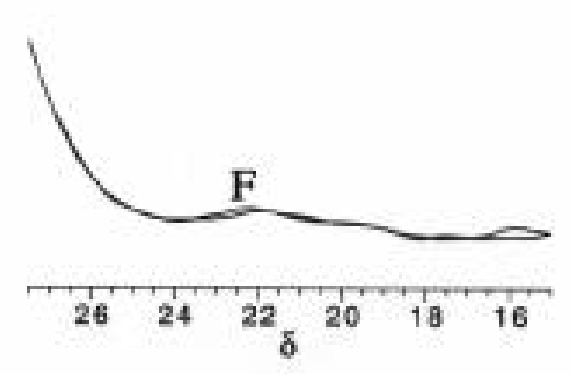


with the PC and combine it with the knowledge that the TTA nitrogens reside more than $6.5 \mathrm{~A}^{0}$ from the PC, the stacking picture proposed by Hewitt, and illustrated in Figure 4.1.6, may have some merit.

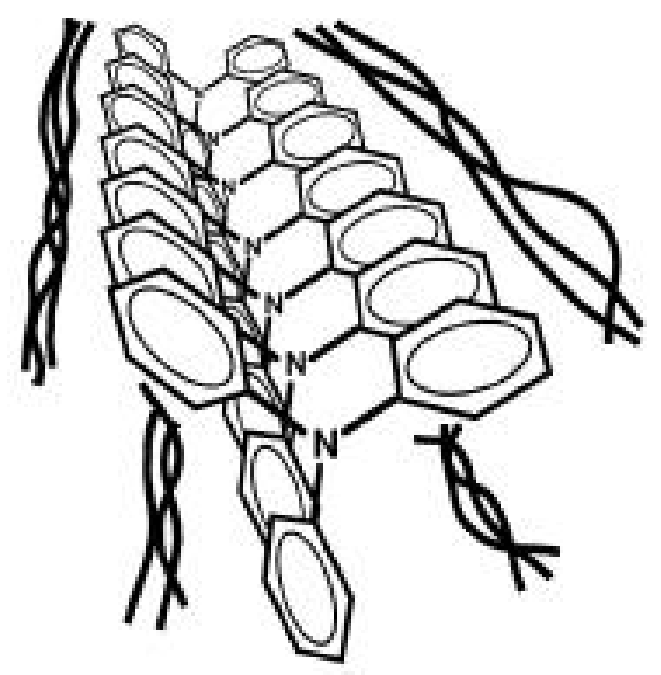

Figure 4.1.6. A cartoon representation of the TTA stacking model proposed by Hewitt. The curved line represent $P C$ chains, and methyl groups have been omitted from the TTA molecules for visual clarity.

\subsection{Structural Relationship between TTA Molecules}

It is clear that TTA and PC form a miscible blend. In addition, the ${ }^{13} \mathrm{C}-{ }^{2} \mathrm{H}$ REDOR and ${ }^{13} \mathrm{C}-{ }^{15} \mathrm{~N}$ REAPDOR experiments suggest that the TTA molecules are ordered to some degree relative to one another. The stacking picture is preliminary and is based simply on two pieces of data, the TTA deuterons are in close contact with the PC, while the TTA nitrogen is effectively shielded from the PC carbons. The next step in elucidating the organization of the TTA in the PC matrix is to make distance measurements from one TTA molecule to another.

Consider a blend (70:30 PC:TTA) in which the TTA portion of the blend is comprised of a 1:1 mole ratio of fully deuterated and ${ }^{15} \mathrm{~N}$ labeled TTA $\left(\mathrm{d}_{21}-{ }^{15} \mathrm{~N}-\mathrm{TTA}\right)$ and TTA molecules which have one methyl ${ }^{13} \mathrm{C}$ label $\left({ }^{13} \mathrm{C}(7)\right.$-TTA). The natural abundance of ${ }^{15} \mathrm{~N}$ is $0.3 \%$, so the ${ }^{13} \mathrm{C}$ labeled TTA is mostly absent of ${ }^{15} \mathrm{~N} . \quad \mathrm{A}^{13} \mathrm{C}-{ }^{2} \mathrm{H}$ REDOR 
experiment will give information about the proximity of TTA molecules relative to one another. Figure 4.2.1 shows the ${ }^{13} \mathrm{C}-{ }^{2} \mathrm{H}$ REDOR data collected for this blend.

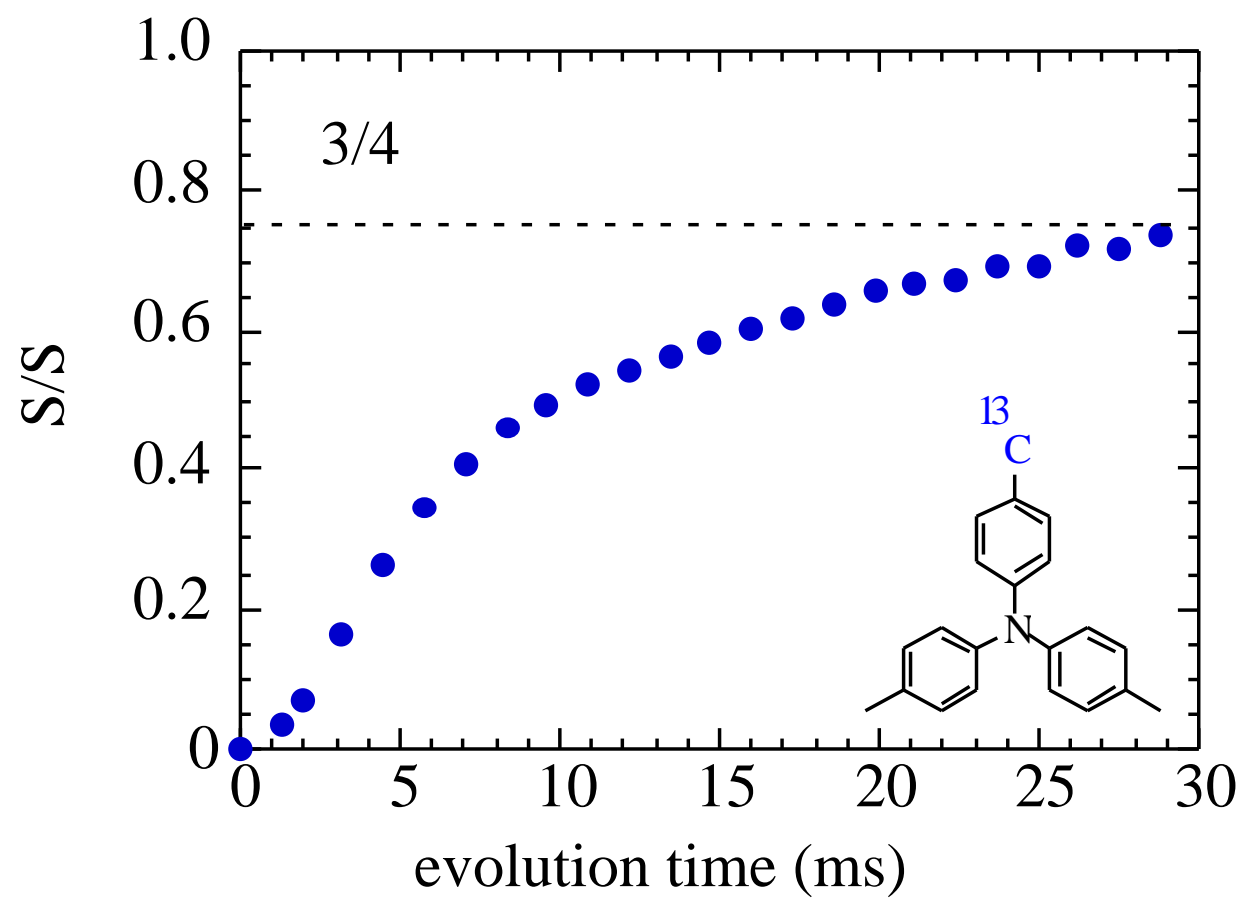

Figure 4.2.1. ${ }^{13} \mathrm{C}-{ }^{2} \mathrm{H}$ REDOR data measuring proximity of one TTA to another. Data is for the methyl carbon of TTA.

The ${ }^{13} \mathrm{C}$ dipolar dephasing tends toward an asymptotic $\frac{\Delta S}{S}$ limit of 0.75 instead of 1.0. Consider a chain of TTA molecules consisting of a 1:1 mole ratio of $\mathrm{d}_{21}{ }^{-15} \mathrm{~N}$-TTA and ${ }^{13} \mathrm{C}(7)$-TTA. In a randomly assembled, one-dimensional chain of these TTA molecules, there is a $50 \%$ chance that a neighboring TTA molecule is fully deuterated. It follows that each of the methyl ${ }^{13} \mathrm{C}$ labeled TTA molecules will have a $75 \%$ chance of having one or both of the neighboring TTA molecules fully deuterated. The data in Figure 4.2.1 from the 1:1 TTA blend agrees with the chain theory by reaching the predicted $\Delta \mathrm{S} / \mathrm{S}$ reaches a limit of 0.75. This is a very fortunate result, as a higher limit for our $\Delta \mathrm{S} / \mathrm{S}$ value might be obtained if the proposed chains were bundled together (see section 4.3), allowing the ${ }^{13} \mathrm{C}$ methyl carbons to be dephased by the $\mathrm{d}_{21}{ }^{-15} \mathrm{~N}$-TTA molecules of neighboring chains rather than by the $\mathrm{d}_{21}{ }^{-}{ }^{15} \mathrm{~N}$-TTA molecules within the same chain. 
The ${ }^{13} \mathrm{C}-{ }^{2} \mathrm{H}$ REDOR data is highly suggestive that there is some type of organization for the TTA molecules in the PC matrix and, whatever the structure, the TTA molecules do not follow the uniform dispersion model. A stacking model has been proposed for the TTA structure based on previous research, and three pieces of data from this lab support that picture: 1) the nitrogens of the TTA molecules are shielded (more than $6 \stackrel{\circ}{A}$, the radius of a TTA molecule) from the carbons of the PC 2) the deuterons of the deuterated TTA molecules are in intimate contact with the carbons of the PC and 3) there is strong evidence that the TTA molecules form one dimensional chains in the PC.

A more detailed picture of the TTA-TTA structural relationship requires intermolecular measurements between specific TTA sites. Because of the low natural abundance of ${ }^{13} \mathrm{C}$, labeling a site-specific ${ }^{13} \mathrm{C}$ will increase the corresponding NMR signal by a factor of 100. Consider a TTA/PC blend in which half of the TTA molecules have one ${ }^{13} \mathrm{C}$ label on one of the tolyl positions and the other half of the TTA molecules are fully deuterated and labeled with a ${ }^{15} \mathrm{~N}$. A ${ }^{13} \mathrm{C}-{ }^{15} \mathrm{~N}$ REDOR experiment will yield dipolar information concerning the distance between the ${ }^{13} \mathrm{C}$ labeled tolyl position and the ${ }^{15} \mathrm{~N}$ of the neighboring TTA.

Figure 4.2.2 shows the results of ${ }^{13} \mathrm{C}-{ }^{15} \mathrm{~N}$ REDOR measurements for the methyl carbon of TTA in a blend in which half the TTA molecules are ${ }^{13} \mathrm{C}(7)$-TTA, and the other half are $\mathrm{d}_{21}{ }^{15} \mathrm{~N}$-TTA. The REDOR data shown in Figure 4.2.2 clearly show that the nitrogen sites of one molecule are near the methyl sites of a neighboring TTA. Based on the stacking model, we would expect the methyl carbon to be the farthest tolyl carbon from a neighboring TTA nitrogen. In order to confirm this, a series of 70:30 PC:TTA blends was prepared with ${ }^{13} \mathrm{C}$ labels at different positions on the TTA. In each of the blends, the TTA component is a 1:1 mixture of $\mathrm{d}_{21}{ }^{-15} \mathrm{~N}$-TTA and a TTA with a ${ }^{13} \mathrm{C}$ label at position of either $\mathrm{A}, \mathrm{B}, \mathrm{B}$ ', or $\mathrm{C}$ as shown in Figure 4.2.3. ${ }^{13} \mathrm{C}-{ }^{15} \mathrm{~N}$ REDOR experiments are performed on each blend to probe the distance from the ${ }^{13} \mathrm{C}$ site of TTA to the ${ }^{15} \mathrm{~N}$ site of a neighboring TTA. 


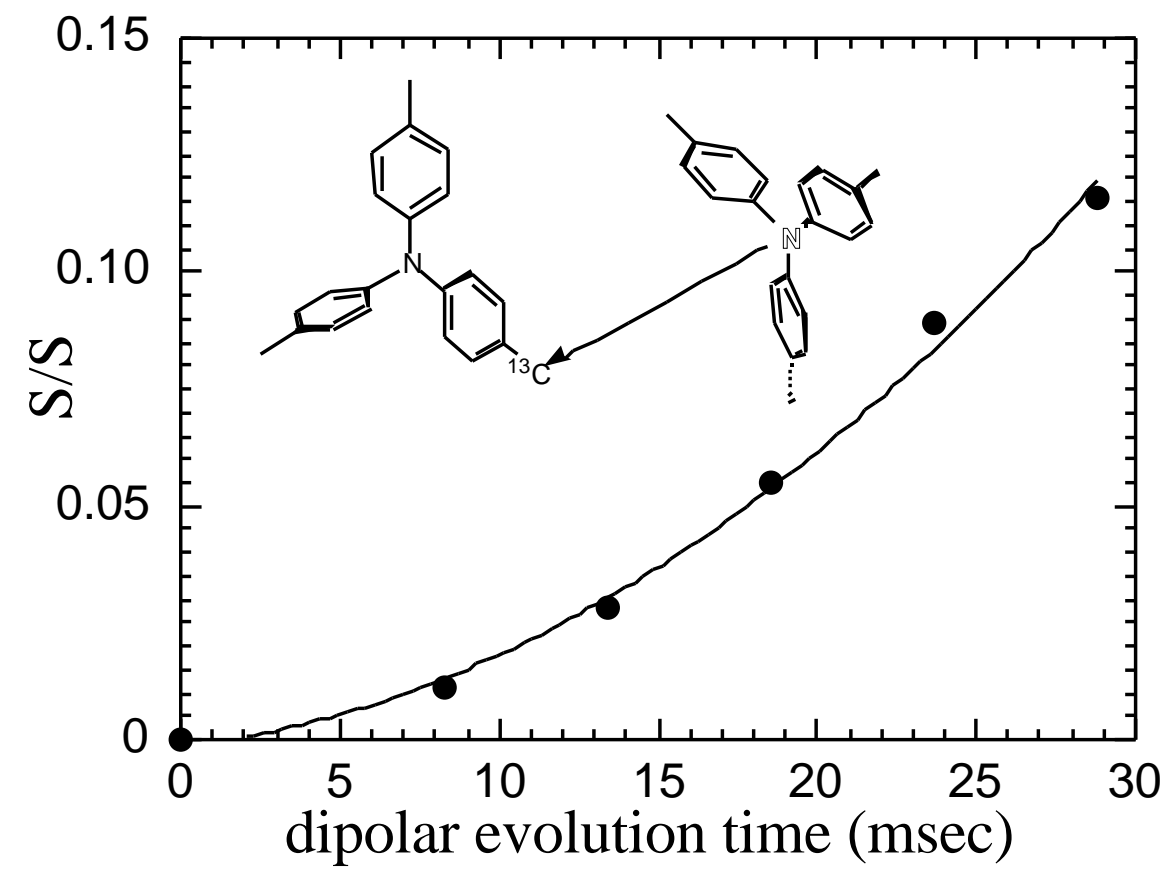

Figure 4.2.2 shows ${ }^{13} \mathrm{C}^{-13} \mathrm{~N}$ REDOR data measuring the proximity of a TTA methyl site to the ${ }^{15} \mathrm{~N}$ of a neighboring TTA.

The data from this series of blends is presented in Figure 4.2.3.

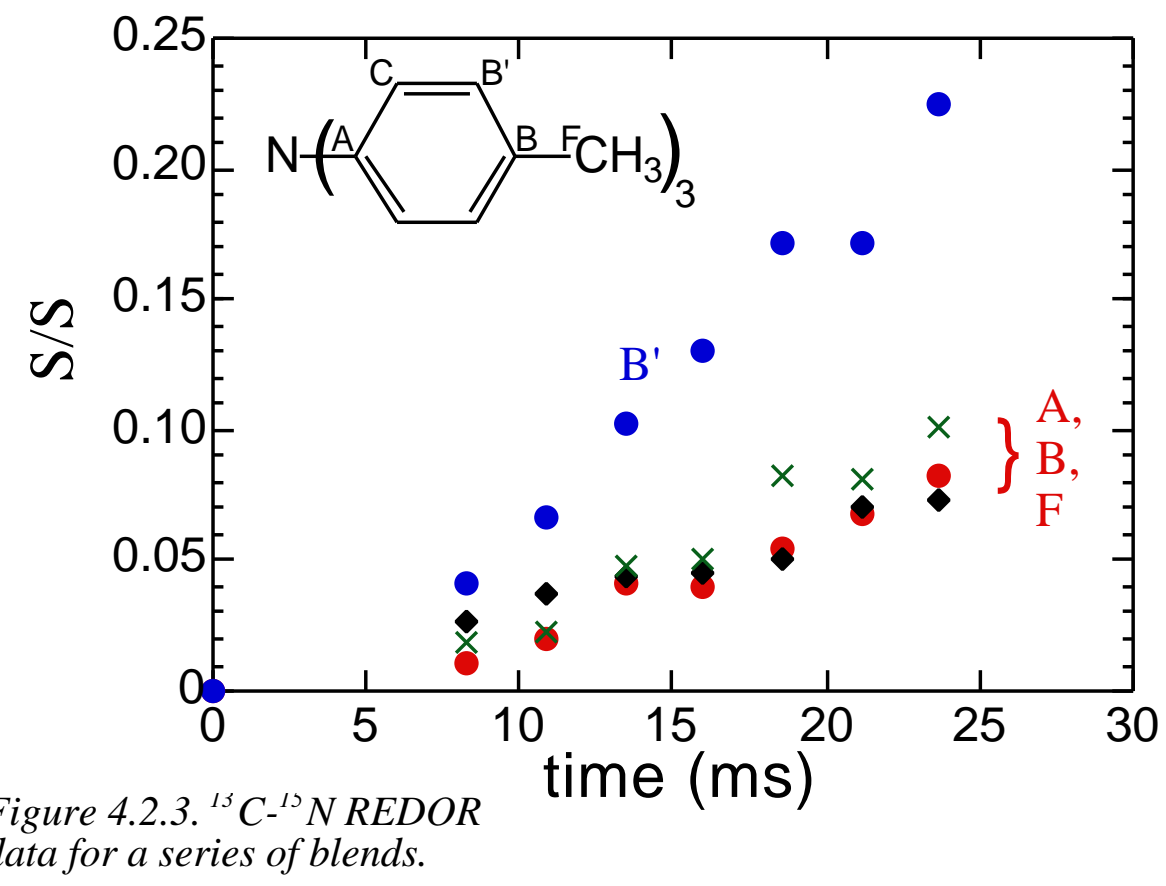


The data in Figure 4.2.3 appears to contradict the stacking model. According to the data from this series of experiments, the B' carbon is closest to the nitrogen of the neighboring TTA molecules since it has higher values of $\Delta \mathrm{S} / \mathrm{S}$ at all times. Sites $\mathrm{A}, \mathrm{B}$, and $\mathrm{F}$ are nearly equivalent in distance from the neighboring nitrogen since they have comparable $\Delta \mathrm{S} / \mathrm{S}$ values. In order to gain a better picture of the arrangement of the TTA molecules, the crystal structure of pure TTA, available on the Cambridge Crystal Database (CCDB), may provide some insight.

Figure 4.2.4 shows an expansion of the TTA crystal lattice along the $\mathrm{c}$ axis. Focusing on the TTA highlighted in red, it is apparent that the protons ortho to the methyl carbon point directly into the tolyl rings of the adjacent TTA. Having the TTA molecules fixed in this way leads to an interesting consequence.

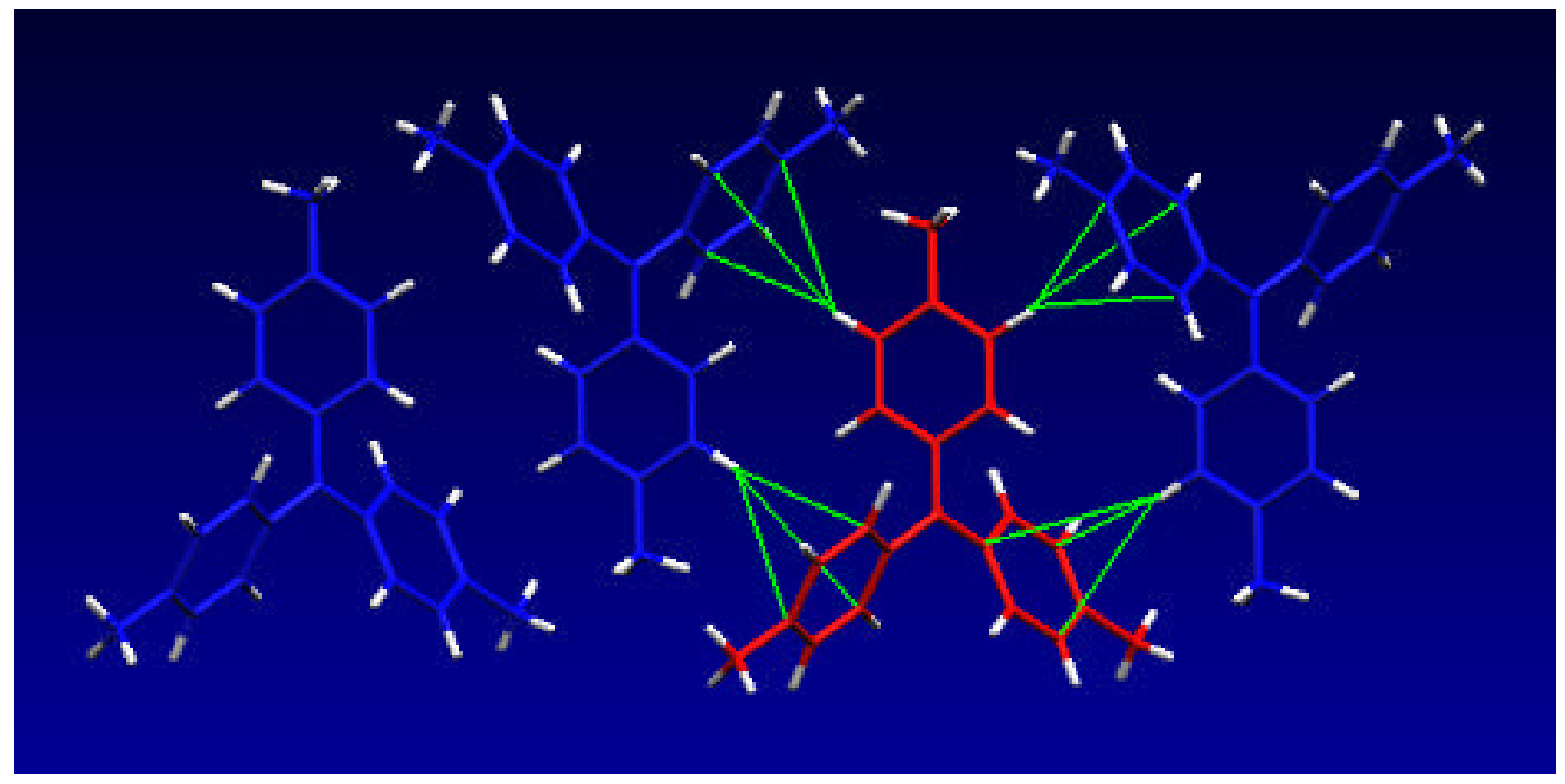

Figure 4.2.4. Crystal structure of TTA along c-axis to illustrate $C H-\pi$ interactions. Adapted from Cambridge Crystal Database and S.L. Reynolds and R.P. Scaringe, Cryst. Struct. Comm. 11, 1989, 1129

The distance from the tolyl carbons of the red TTA to the nitrogens of the neighboring TTA molecules is closest for the carbon at the B' position, as shown in Figure 4.2.5. The 
arrangement of TTA molecules along the c-axis is more or less edge-to-edge and not face to face as proposed in the model proposed by Hewitt.

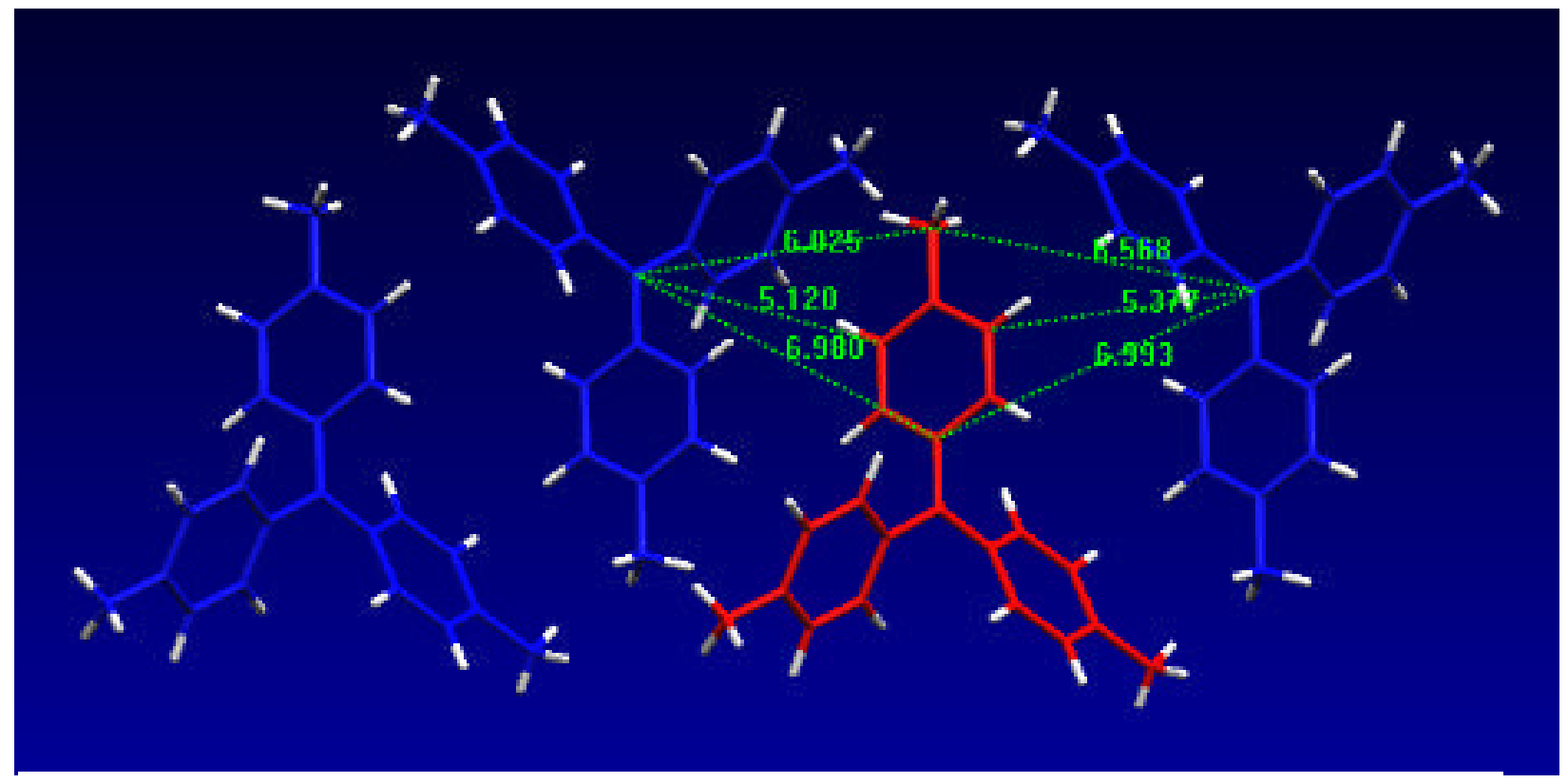

Figure 4.2.5. Crystal structure of TTA along c-axis measuring carbon to nearest neighbor nitrogen distances. Adapted from Cambridge Crystal Database and S.L. Reynolds and R.P. Scaringe, Cryst. Struct. Comm. 11, 1989, 1129

The stacking model first proposed does not appear to be entirely valid, and the REDOR data suggests a new model. It would appear as though the TTA molecules align in the PC matrix in a similar way that they align in their crystal structure along the c-axis. One question that may arise is whether the TTA molecules are simply interacting with one another due to $\mathrm{CH}$-pi interactions, or whether the TTA molecules are beginning to crystallize. $\mathrm{CH}$-pi interactions are well documented and are similar in nature to hydrogen bonding, although the $\mathrm{CH}$-pi interactions are a weaker than the hydrogen bonding. The idea that the TTA is beginning to crystallize may be valid, because TTA will phase separate from the PC matrix once the blend reaches a TTA composition of $40 \mathrm{wt} \%$. An important question, then, is at what concentration does organization initiate? 


\subsection{Onset of Ordering}

The REDOR data suggests that TTA molecules form edge-to-edge chains. The onset of the ordering of the TTA molecules may be found by lessening the amount of TTA in the blend. Figure 4.3.1 shows REDOR data from three different dTTA/PC blends. The TTA portion of each blend contains an equimolar amount of $\mathrm{d}_{21}{ }^{-}{ }^{15} \mathrm{~N}-\mathrm{TTA}$ and ${ }^{13} \mathrm{C}(7)$-TTA. The total weight percent of TTA in the blend is varied between 5\% and $30 \%$.

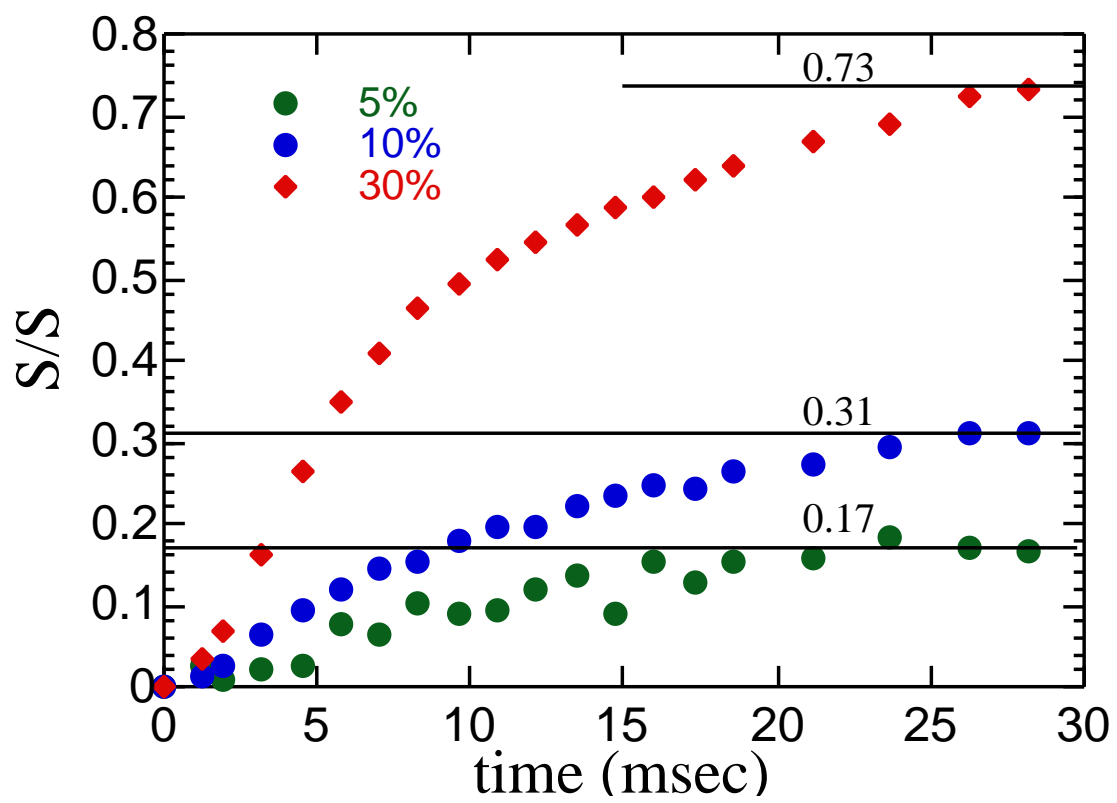

Figure 4.3.1. $\Delta S / S$ limits indicate the degree of ordering for blends with varied wt\% of TTA

In a $70: 30$ blend the $\Delta \mathrm{S} / \mathrm{S}$ reaches a limit of 0.73 . According to the chain model, this would indicate that there is $100 \%$ organization in the $70: 30$ blend. Comparison of the asymptotic limits of $\Delta \mathrm{S} / \mathrm{S}$ for the different wt $\%$ dTTA samples shows that, with as little as $5 \mathrm{wt} \%$ TTA, $23 \%$ of the TTA molecules are arranged in the proposed chain structure. When the TTA molecules make up $10 \%$ of the blend, $42 \%$ of the TTA has organized into the chain structure. It was mentioned earlier that with the uniform dispersion model, the TTA molecules would be separated by an average distance of $12 \stackrel{\circ}{A}$ for the $30: 70$ blend. In a 95:5 PC:TTA blend, the TTA molecules would be separated by an average of $21 \stackrel{\circ}{\mathrm{A}}$ if they 
followed the uniform dispersion model. The sum of the REDOR data suggests that TTA is organized into linear strands and that the onset of ordering occurs for samples with as little as $5 \mathrm{wt} \%$ TTA. Are these strands isolated from one another or are they clustered together, as depicted in Figure 4.3.2? In Figure 4.3.2, the N's represent the nitrogen of a TTA and the black dots represent the position of the methyl groups on the TTA molecule. Each cartoon TTA represents the end of a linear chain of TTA molecules held in a PC matrix, with the PC represented by the curved lines. Consider the two packing structures of Figure 4.3.2. In the scheme on the left, the chains are clustered around one another, possibly on the verge of phase separation, while in the scheme on the right, the chains are separated by of the
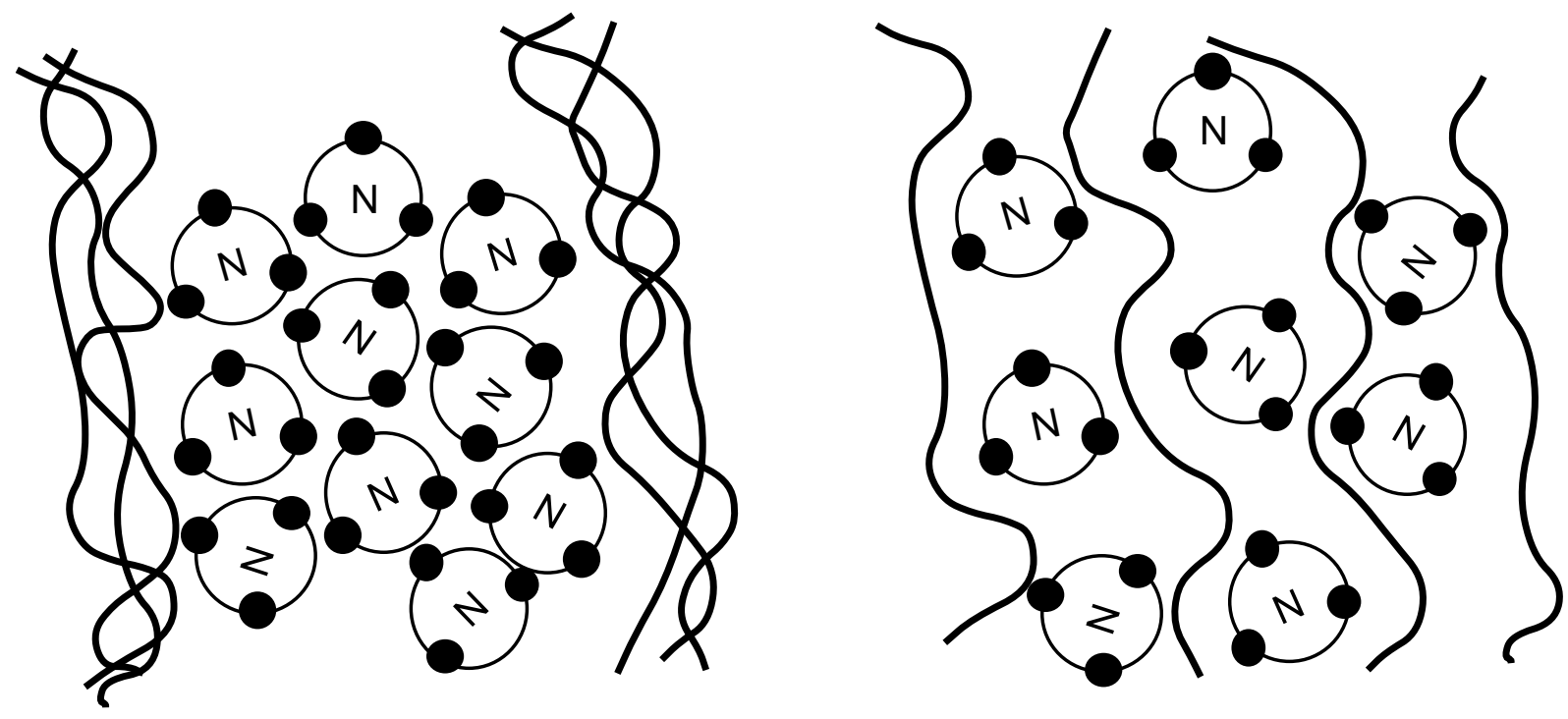

Figure 4.3.2 shows two possible arrangements for chains of TTA molecules in PC.

polymer. Nearly all of the TTA methyl groups would be in contact with the polymer in the isolated chain model. However, in the clustered chain model, many of the methyl carbons would be shielded from the polymer. In order to differentiate between these two possibilities, a blend in which the PC is deuterated and the TTA has a single ${ }^{13} \mathrm{C}$ methyl label was prepared. The deuterated PC was a gift from the Kodak Corporation. $\mathrm{A}^{13} \mathrm{C}-{ }^{2} \mathrm{D}$ REDOR experiment was performed to investigate the contacts between the methyl groups of the TTA molecules and the deuterons of the PC. The results of this experiment are 
shown in Figure 4.3.3. The data shows that $84 \%$ of the TTA methyl groups are in intimate contact with the PC. In the isolated chain model, $\Delta \mathrm{S} / \mathrm{S}$ should tend to 1 . The data goes to a limiting value of 0.84 , which suggests that most of the strands are isolated, but that clustering may be starting to occur for $30 \mathrm{wt} \%$ blends.

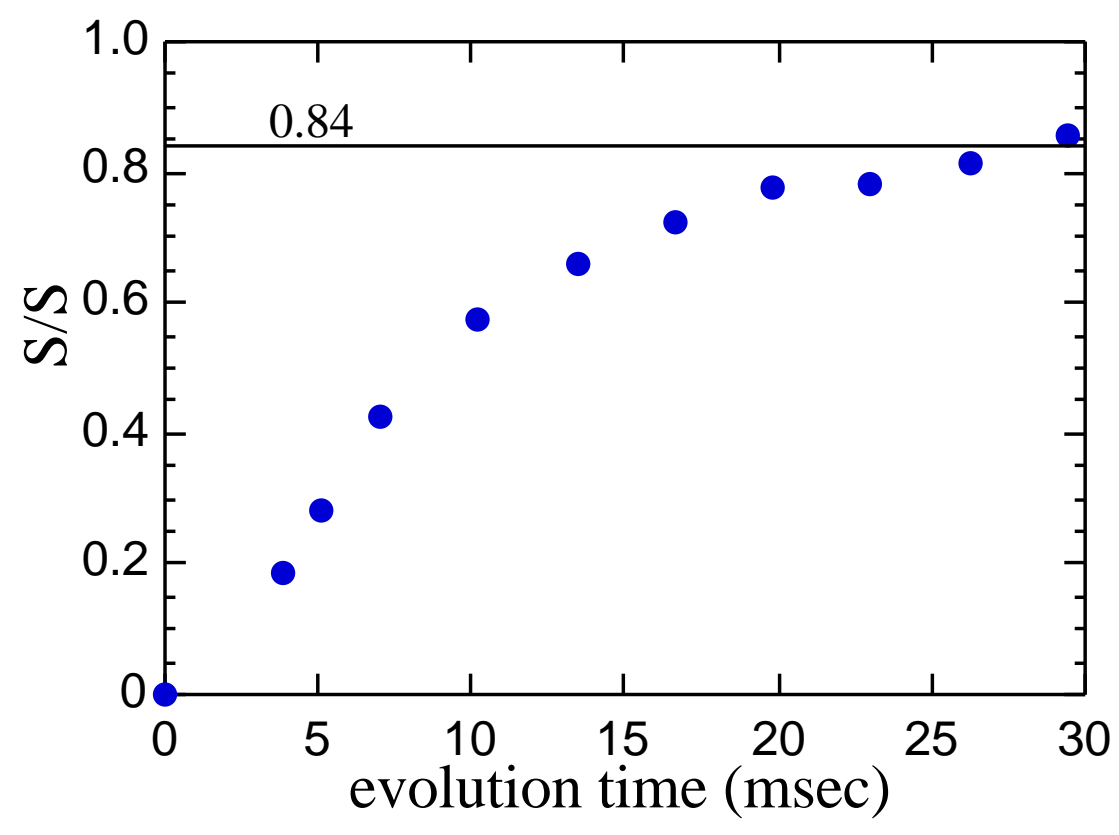

Figure 4.3.3 ${ }^{13} \mathrm{C}_{-}^{2} \mathrm{D}$ REDOR data from a blend of deuterated $P C$ and methyl ${ }^{13}$ C labeled TTA. 


\section{Chapter 5. Conclusions}

Polymer films doped with photoconductiong organic molecules play an important role in the xerography process. These polymer blends are amorphous systems difficult to characterize with traditional methods of structural elucidation such as x-ray scattering and liquid NMR. While traditional studies involving such blends have assumed that the doped molecules follow a uniform dispersion model, NMR studies begun in 1984 have suggested a model for the organization of the dopant molecules. Modern NMR techniques such as REDOR and REAPDOR, in combination with simple isotope labeling techniques, allow site-specific distance measurements to be made between the dopant molecules and host polymer, as well as between neighboring dopant molecules. These measurements suggest a

more detailed model than previously considered while serving as a complimentary technique to other methods used to characterize amorphous systems such as neutron scattering. As a result of the NMR measurements made on this system, a structure for the organization of tri-p-tolylamine in a polycarbonate matrix is proposed. This model for organization involves the TTA molecules forming one-dimensional chains similar to the organization of the TTA molecules along the c-axis in pure crystalline TTA. Ordering of dopant molecules in polymers is controversial. The NMR data reported here supports an ordered arrangement of TTA in the PC matrix. Low Q neutron scattering experiments may be possible with these blends and such experiments may provide additional information about the structured nature of TTA/PC blends. 


\section{Appendix A}

\section{Rotational-Echo, Double-Resonance Dipolar Dephasing}

\section{$\mathrm{I}=\mathbf{1 / 2}, \mathrm{S}=\mathbf{1 / 2}$}

For a pair of spin-1/2 nuclei separated by a distance $r$, the REDOR dipolar dephasing signal, $\mathrm{s}_{\mathrm{d}}$, for an I-spin coupled to an S-spin is

$$
s_{d}=\cos \omega_{d} \tau .
$$

The rate of dipolar dephasing $\omega_{d}$ is

$$
\omega_{d}(t)= \pm \frac{1}{2} d\left[\sin ^{2} \beta \cos 2\left(\alpha+\omega_{r} t\right)-\sqrt{2} \sin 2 \beta \cos \left(\alpha+\omega_{r} t\right]\right.
$$

where $d=\frac{\gamma_{I} \gamma_{S} \hbar}{r^{3}}$ and provided that the spacing between $\pi$ pulse is $\frac{\mathrm{T}_{\mathrm{r}}}{2}$. The angles $\alpha$ and $\beta$ are the azimuthal and polar angles that define the internuclear vector $\bar{r}$ with respect to the rotor frame. The dipolar dephased signal for a powder sample is obtained by summing over all orientations of $\bar{r}$ and weighting each orientation by $\sin \beta$. The powder sum, $S_{d}$, is

$$
S_{d}=\frac{1}{2 \pi} \int_{0}^{\pi} \int_{0}^{\pi} \cos (\phi) \sin \beta d \alpha d \beta .
$$

In addition to the signal loss by coherent averaging of the dipolar coupling to a nonzero value, signal is also lost through incoherent $T_{2}$ relaxation of the I-spin. $T_{2}$ effects are eliminated from data analysis by taking a control signal, $S_{0}$, and taking the ratio $\frac{S_{d}}{S_{0}}$.

REDOR data is commonly represented as $\frac{S_{d}}{S_{0}}$ or as $\frac{\Delta S}{S}=1-\frac{S_{d}}{S_{0}}$.

\section{$\underline{I=1 / 2, S=1}$}

For $\mathrm{S}=1$, REDOR experiments with ${ }^{2} \mathrm{H}$ are performed with a $\pi / 2$ pulse instead of a $\pi$ pulse. The dipolar dephased signal is the sum over $S_{d}=\frac{1}{6}\left(1+4 \cos \varpi_{d} t+2 \cos ^{2} \varpi_{d} t\right)$ where $\varpi_{d}=2 \sqrt{2} D \sin 2 \beta \sin \alpha$ and $\mathrm{D}=\mathrm{d} / 2 \pi$. 


\section{APPENDIX B NMR Spectra}

\section{General description:}

$\mathrm{X}$ coordinates for all NMR spectra are in parts per million. For compounds with no signal, ie, fully deuterated species, characterization was confirmed by lack of NMR peaks in combination with melting points and synthesis procedures. All spectra are organized by compound as noted below.

B1: Bromotoluene spectra

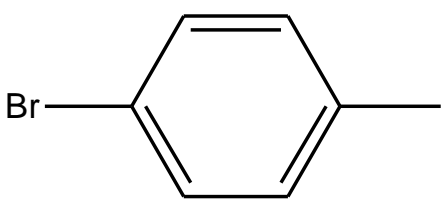

B2: Nitrotoluene spectra

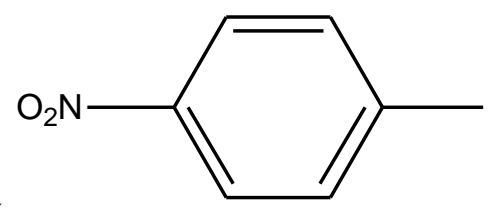

B3: Toluidine spectra

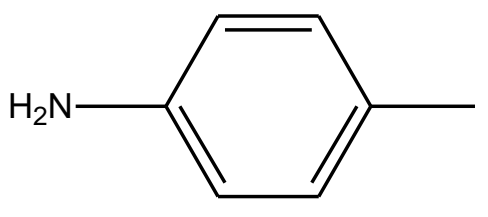

B4: Di-p-tolylamine spectra

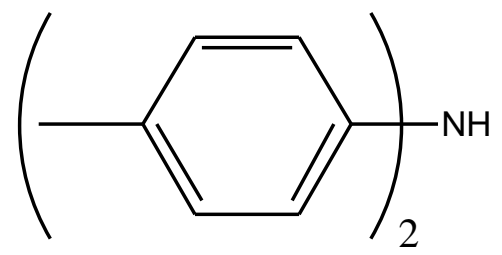

B5: Tri-p-tolylamine spectra

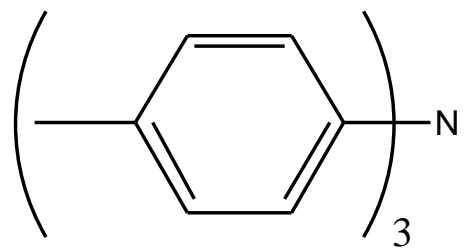


B1.1 $\left({ }^{2} D_{7}\right)$-4-Bromotoluene (1) ${ }^{13} \mathrm{C}$ NMR
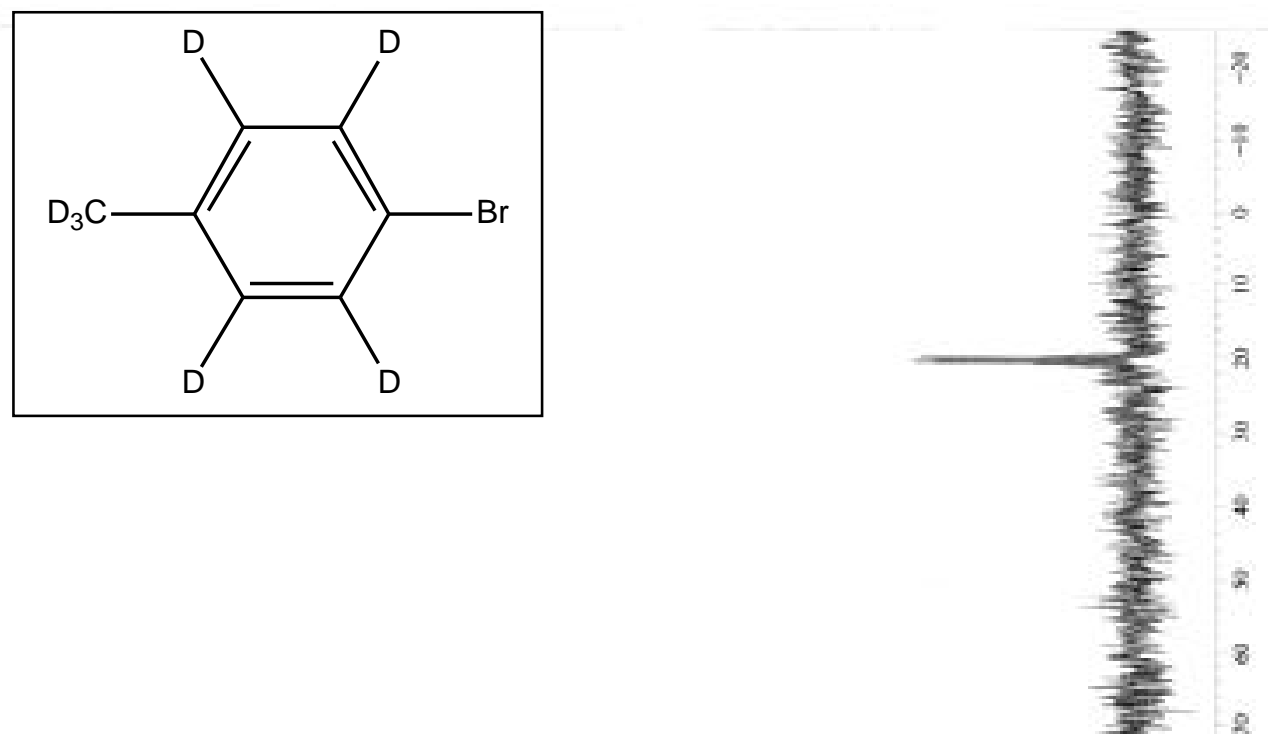


\section{B1.2 $\left({ }^{13} \mathrm{CH}_{3}\right)$-4-Bromotoluene (2) ${ }^{1} \mathrm{H}$ NMR}

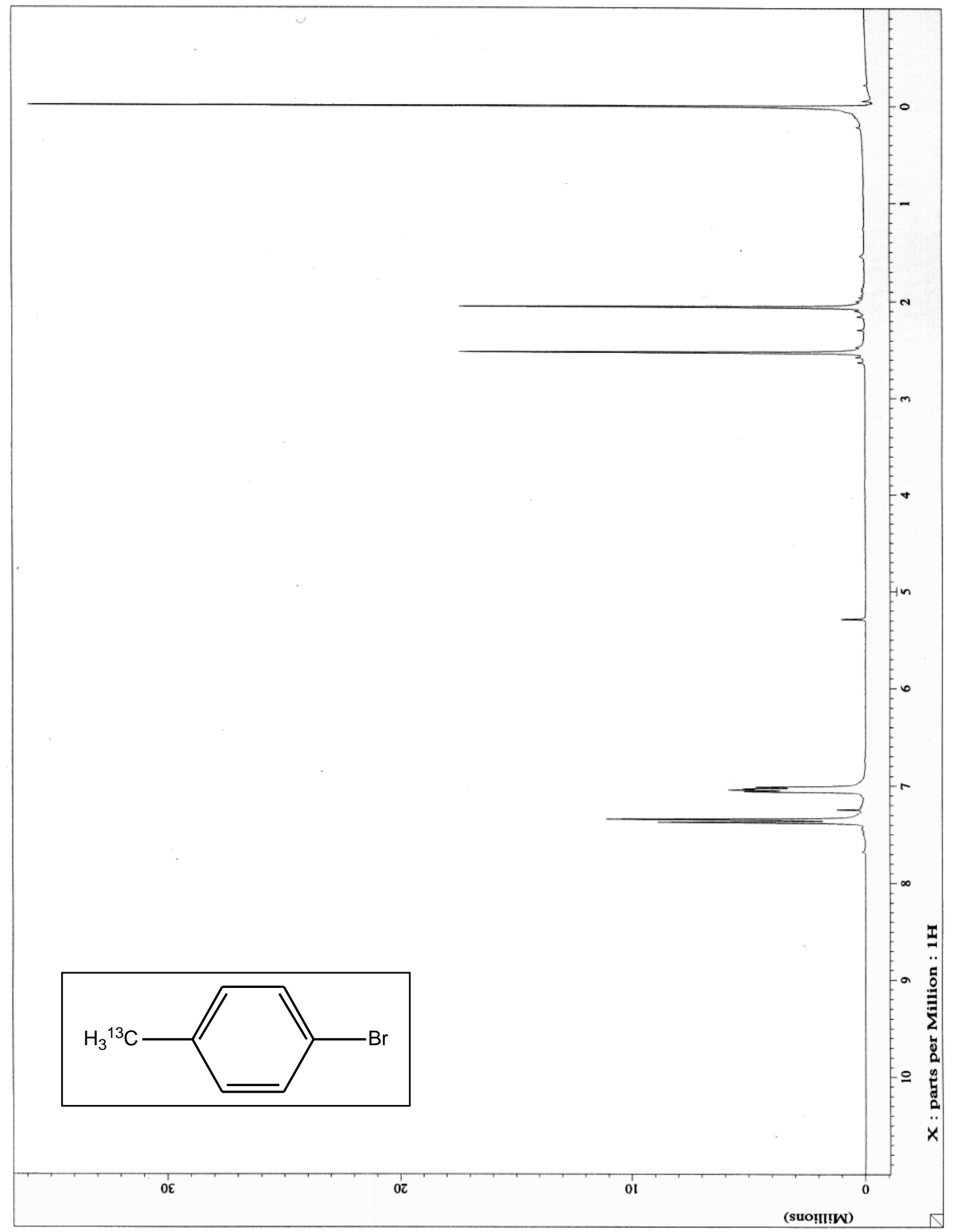




\section{B $1.3\left({ }^{13} \mathrm{CH}_{3}\right)$-4-Bromotoluene (2) ${ }^{13} \mathrm{C}$ NMR}

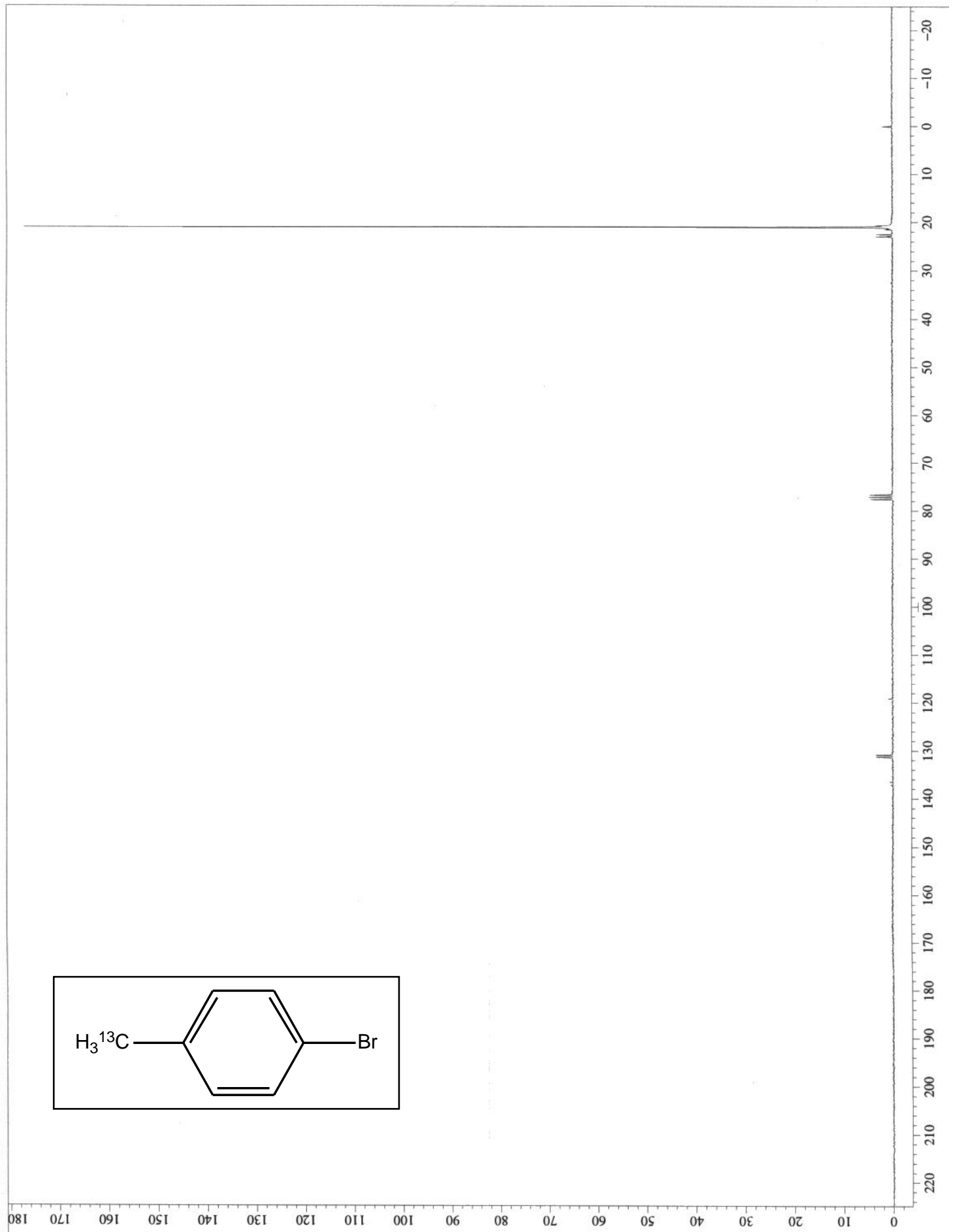




\section{B1.4 ( $\left.C^{2} D_{3}\right)$-4-Bromotoluene (3) ${ }^{1}$ H NMR}

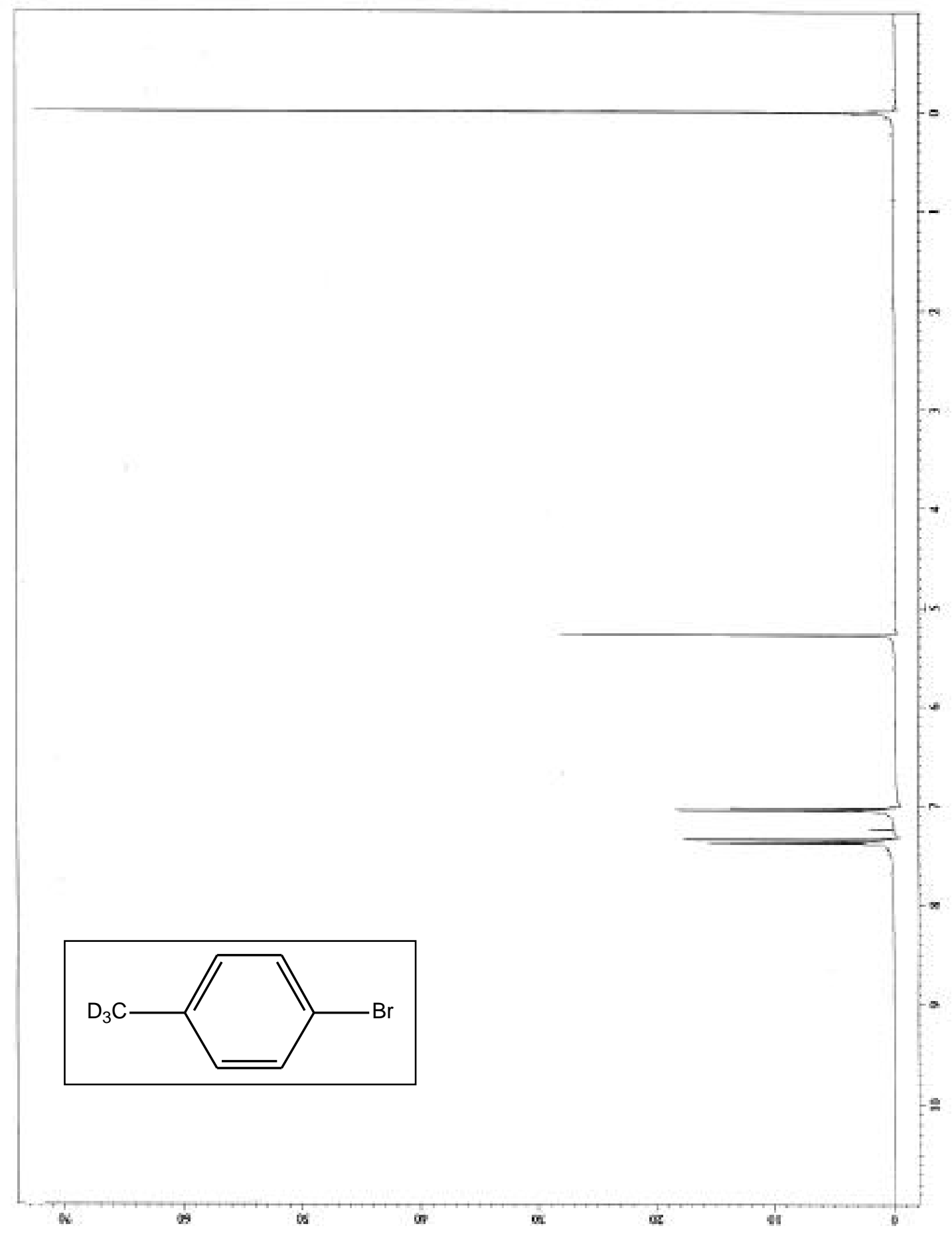




\section{B1.5 $\left(C^{2} D_{3}\right)$-4-Bromotoluene (3) ${ }^{13}$ C NMR}

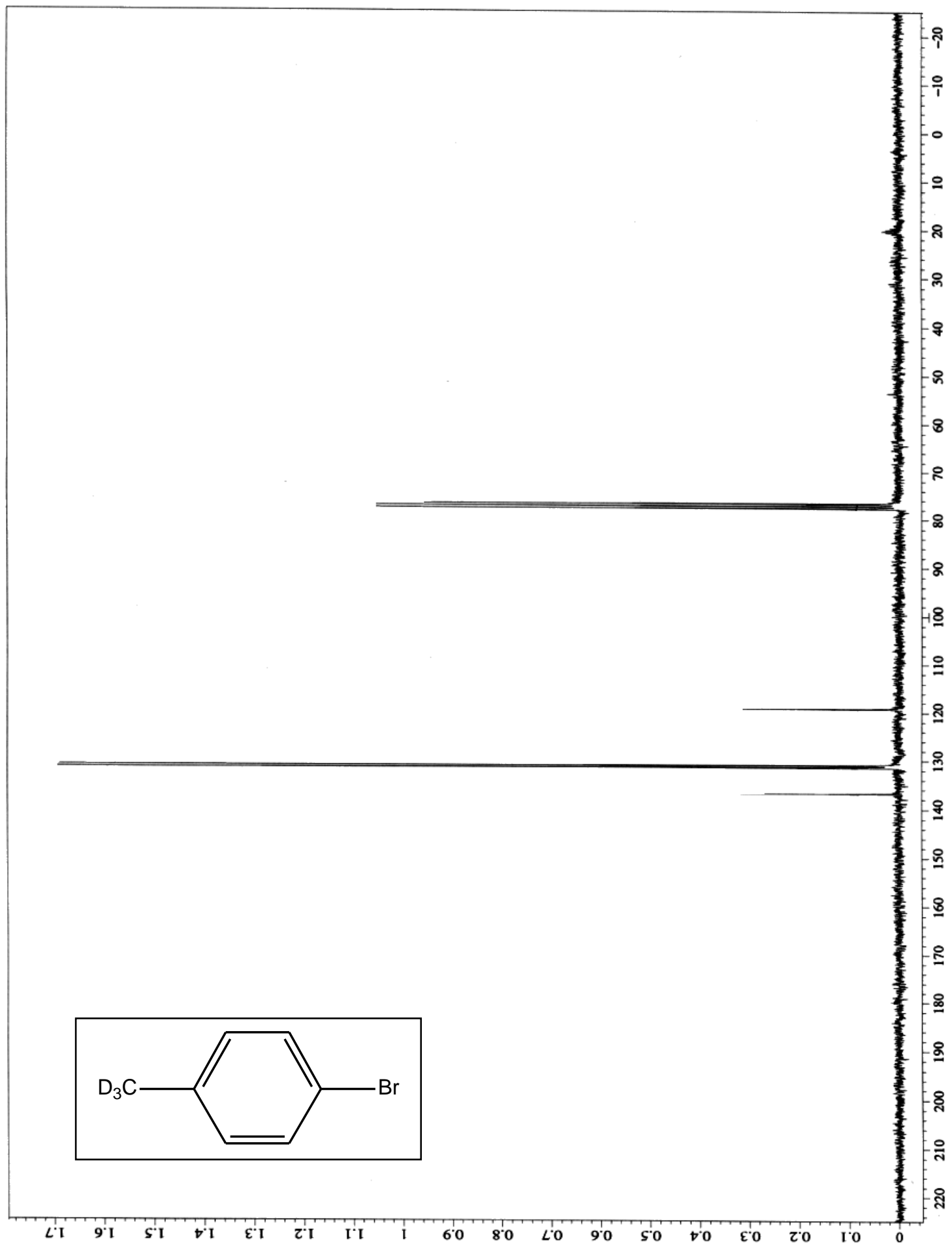


B 2.1 (15N)-4-Nitrotoluene (5) ${ }^{1}$ H NMR

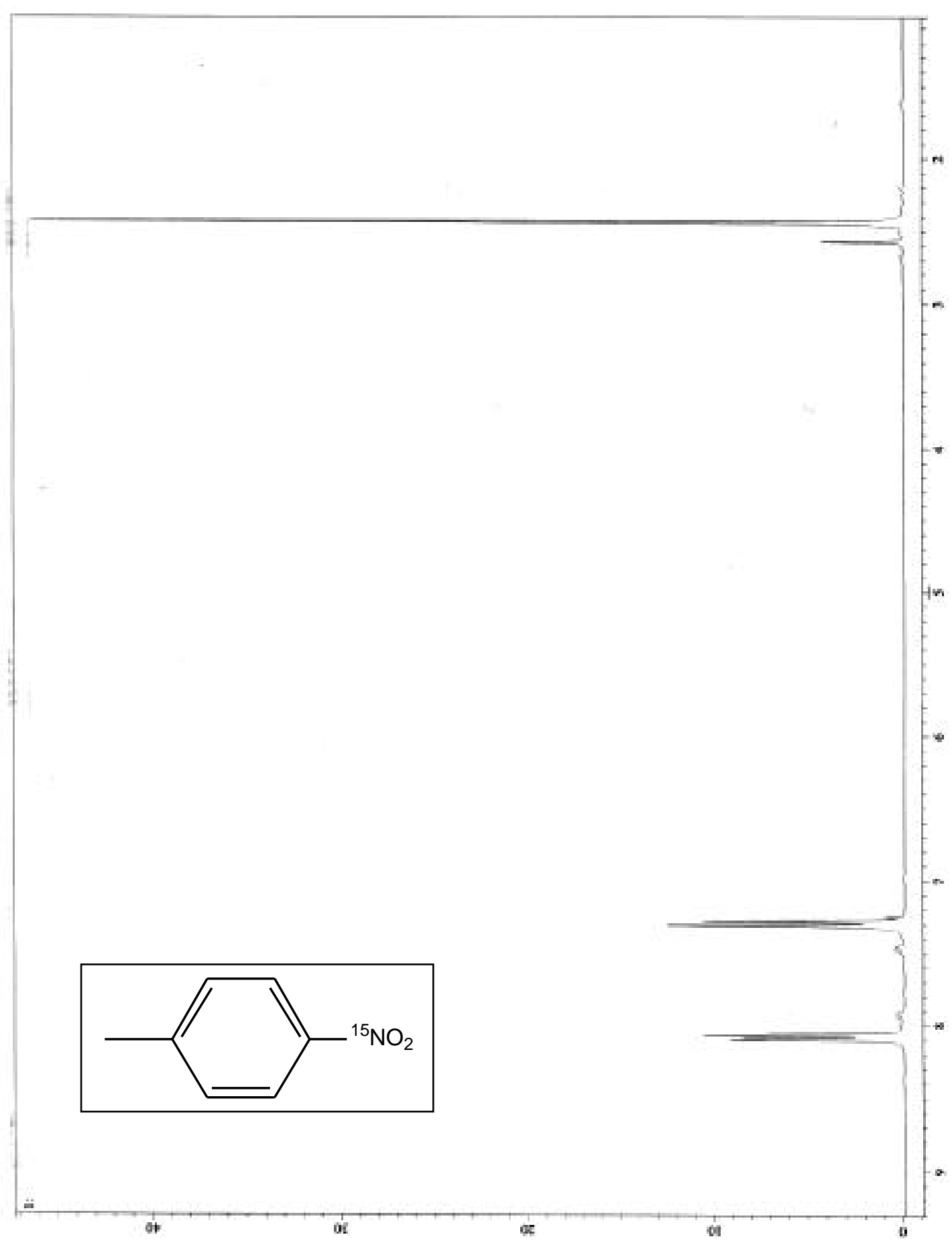


B 2.2 (15N)-4-Nitrotoluene (5) ${ }^{13} \mathrm{C}$ NMR

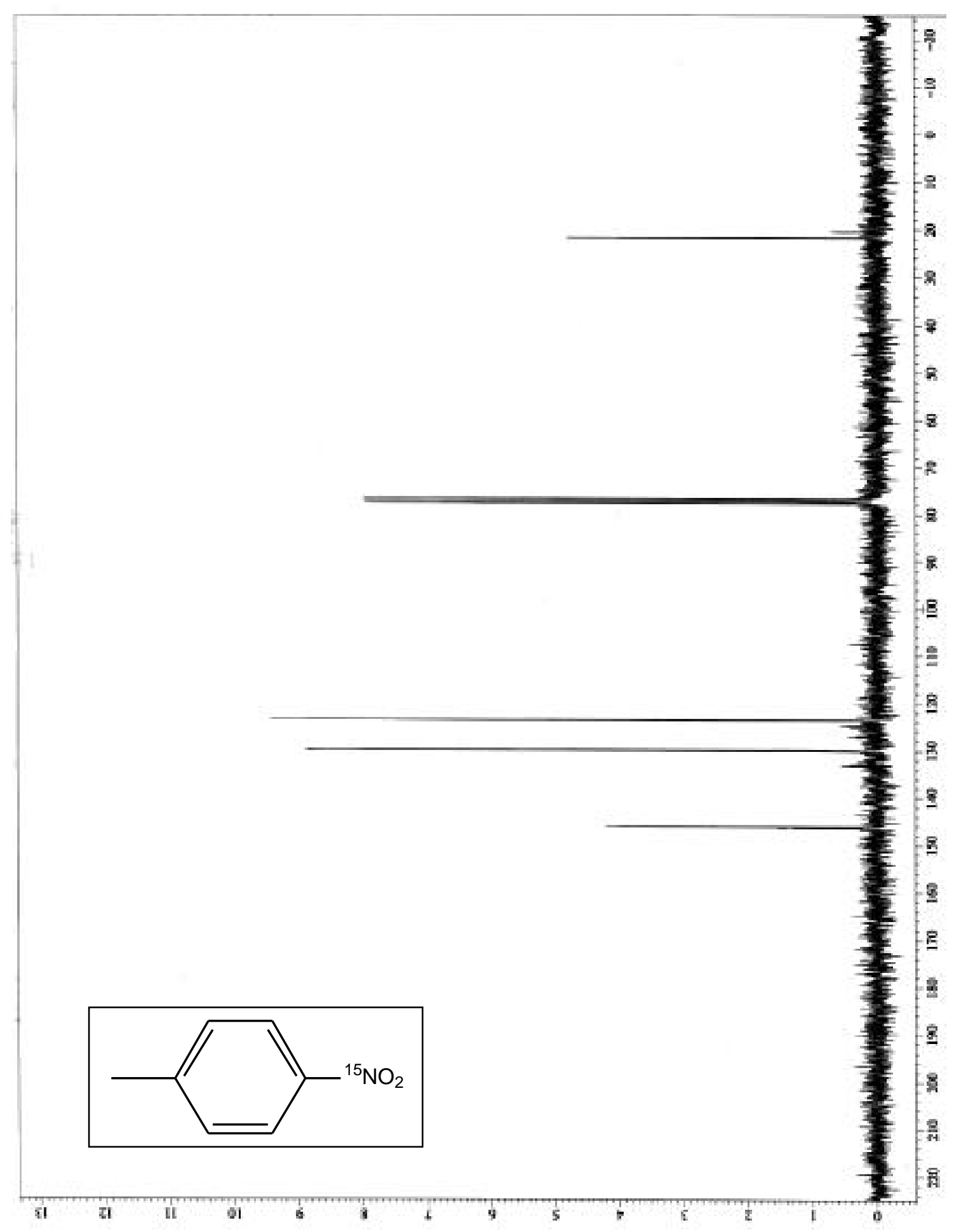

51 
B $2.3\left({ }^{15} \mathrm{~N},{ }^{2} \mathrm{D}_{7}\right)$-4-nitrotoluene (6) ${ }^{1} \mathrm{H}$ NMR

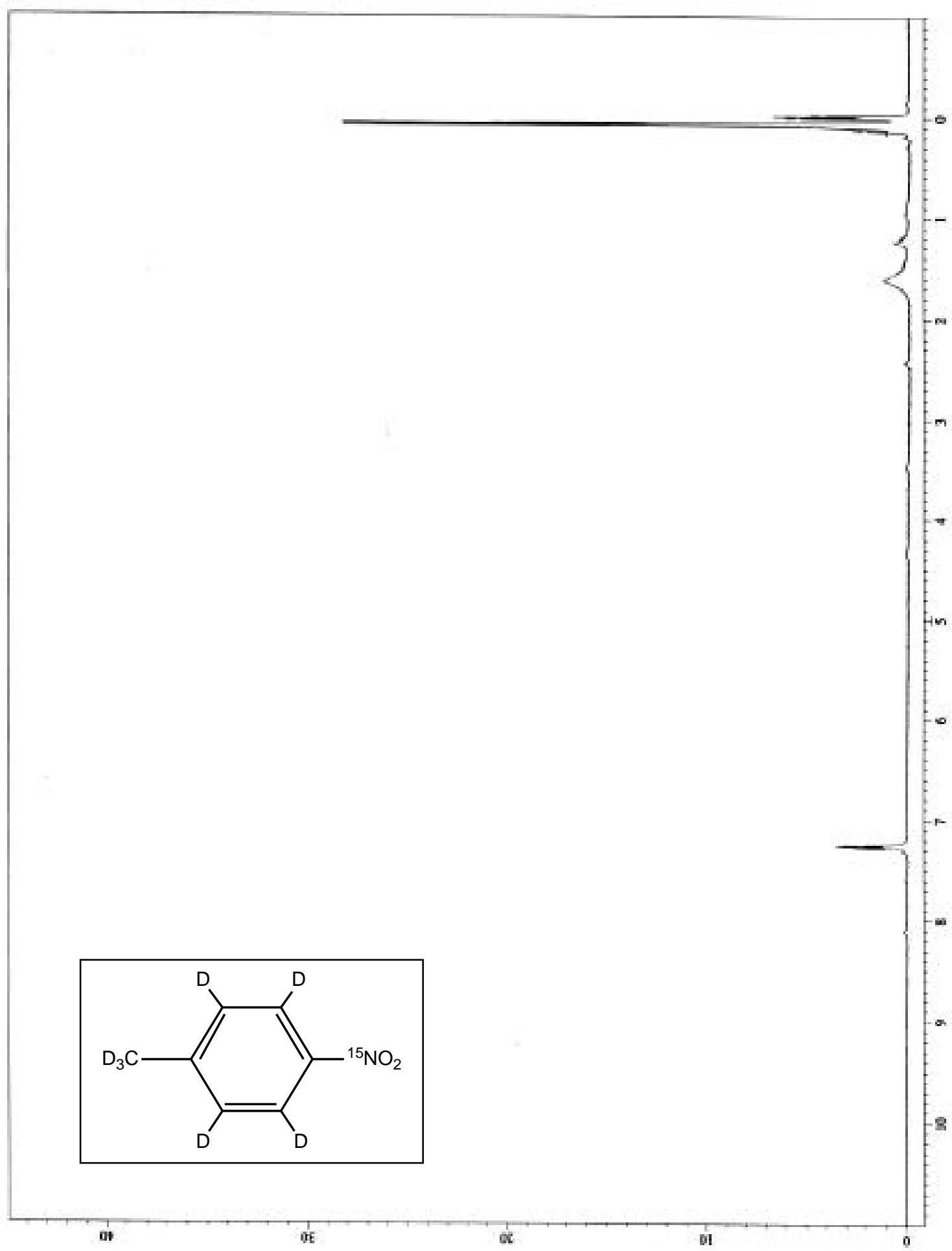


B 2.4 $\left({ }^{15} \mathrm{~N},{ }^{2} \mathrm{D}_{7}\right)$-4-nitrotoluene (6) ${ }^{13} \mathrm{C}$ NMR

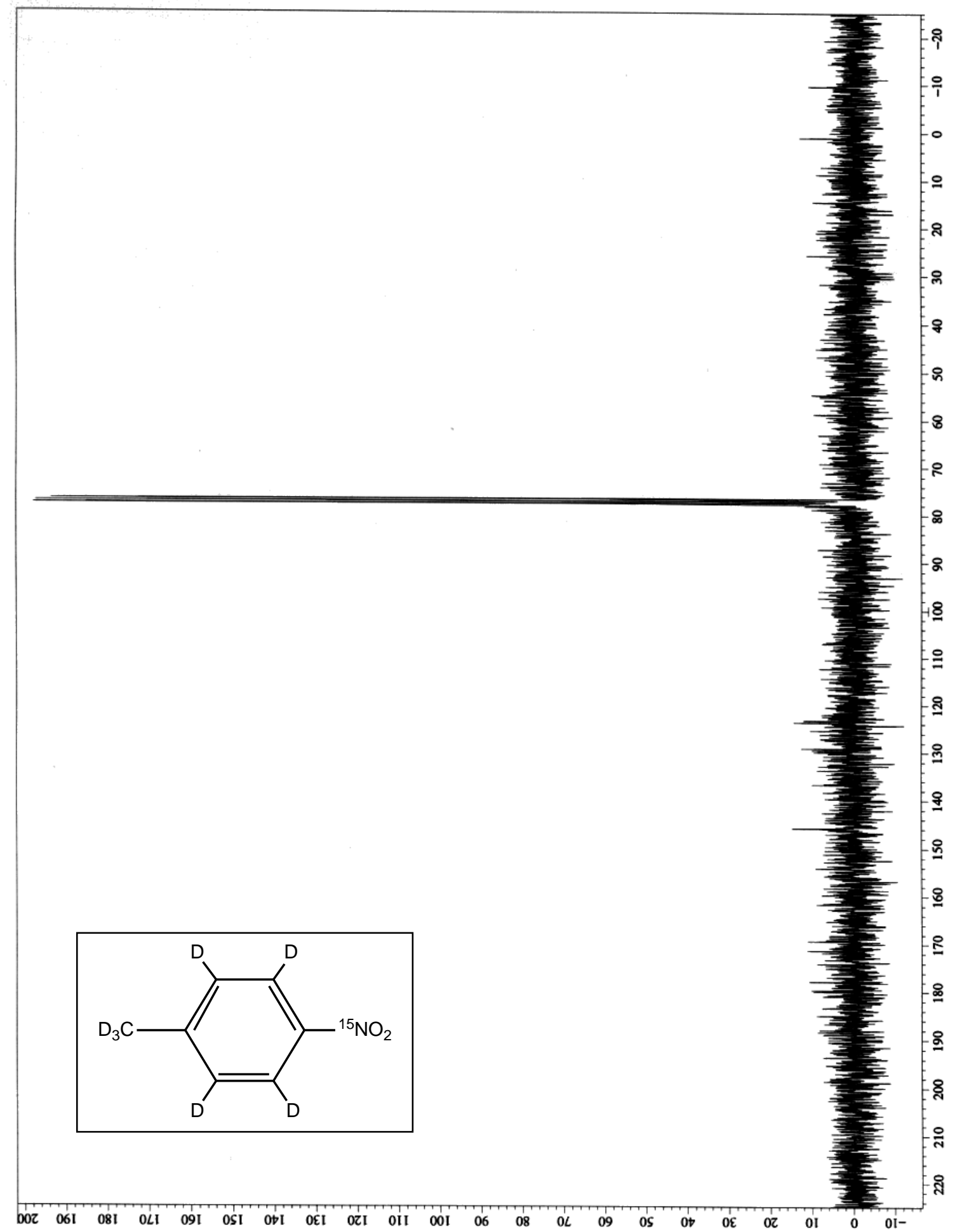




\section{B $3.1\left({ }^{15} \mathrm{~N}\right)$-4-Aminotoluene (7) ${ }^{1} \mathrm{H}$ NMR}

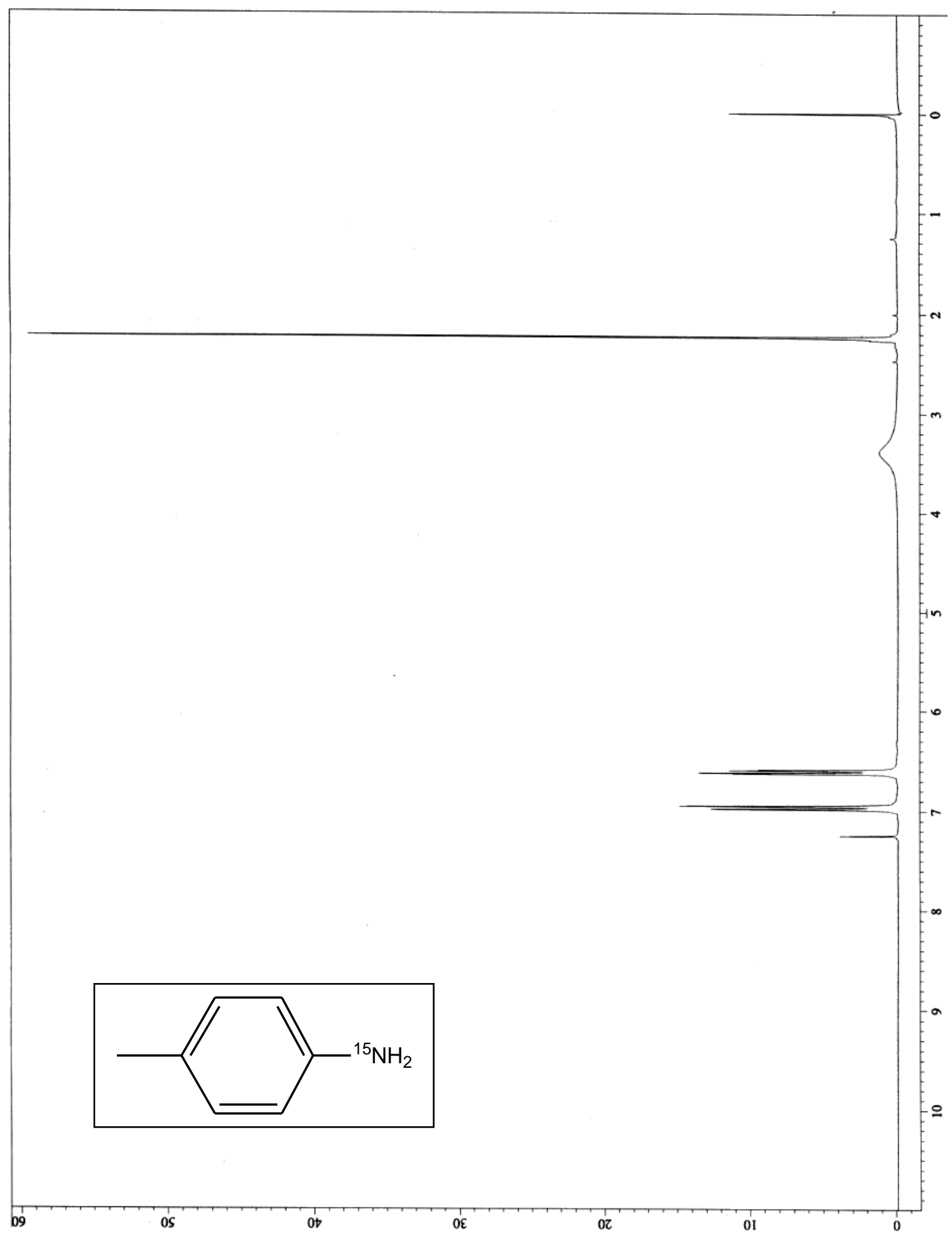




\section{B 3.2 $\left({ }^{15} \mathrm{~N}\right)$-4-Aminotoluene (7) ${ }^{13} \mathrm{C}$ NMR}

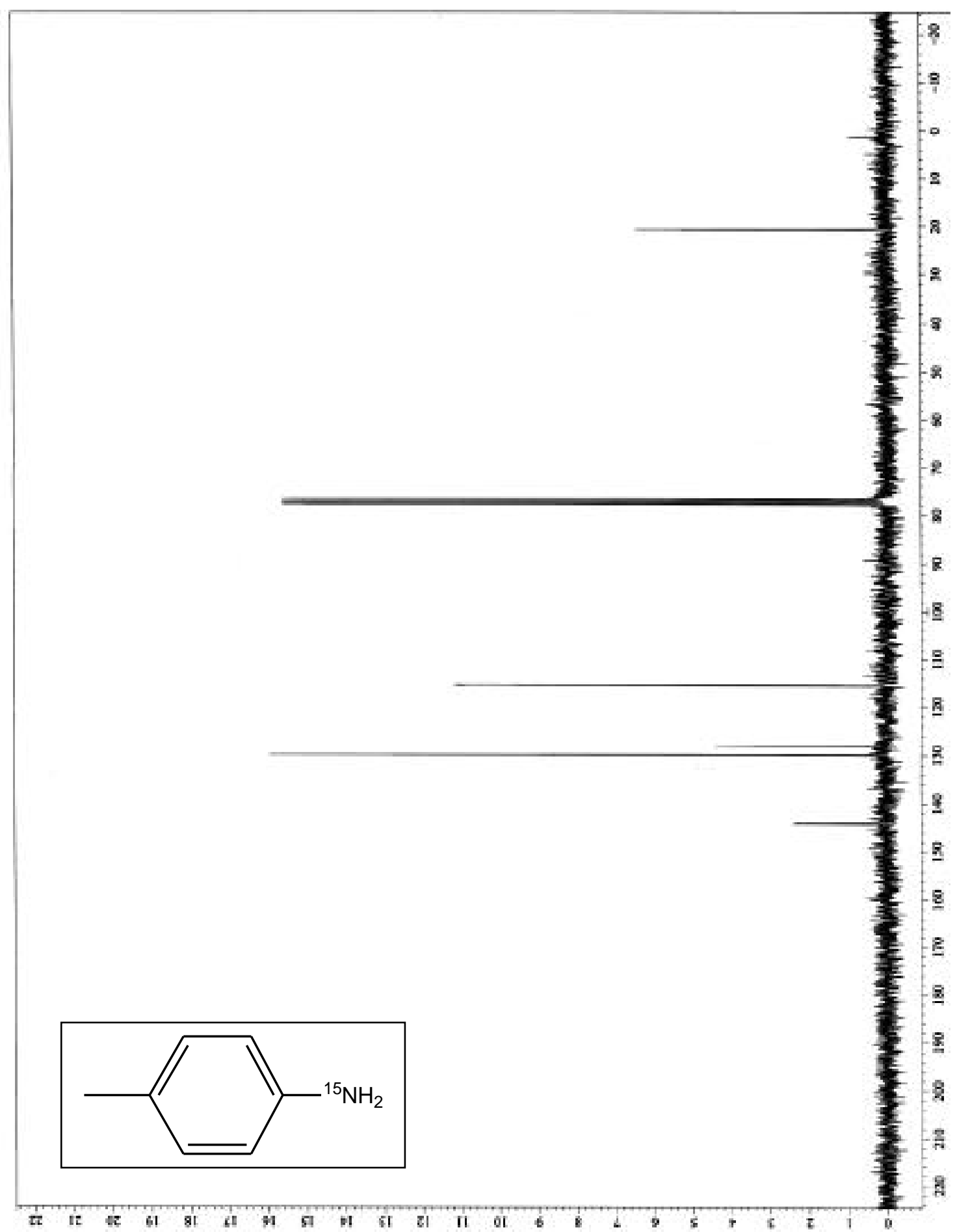


B 3.3 $\left({ }^{15} \mathrm{~N}\right)$-4-Amino- $\left({ }^{2} \mathrm{D}_{7}\right)$-toluene (8) ${ }^{1} \mathrm{H}$ NMR

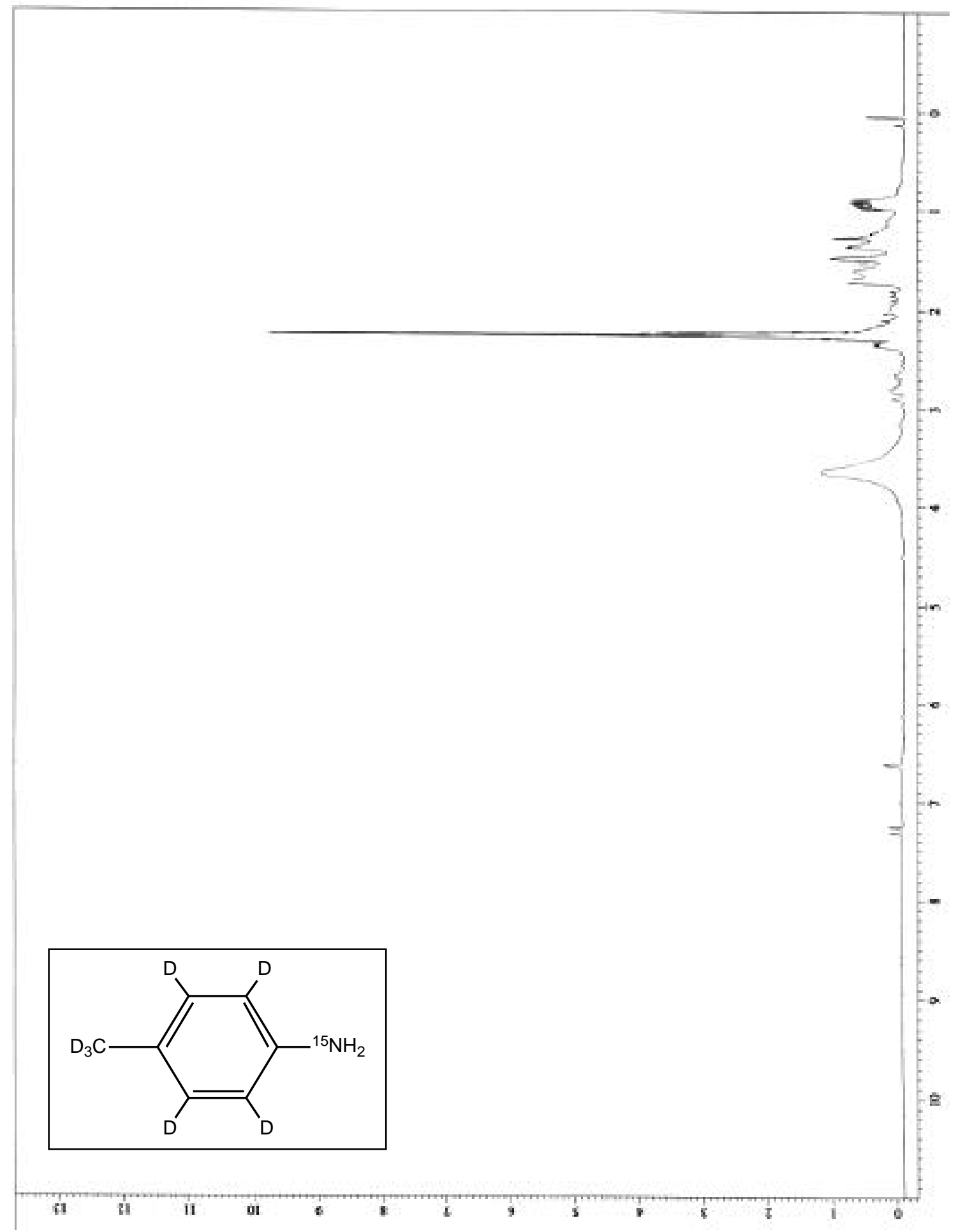


B $3.4\left({ }^{15} \mathrm{~N}\right)$-4-Amino- $\left({ }^{2} \mathrm{D}_{7}\right)$-toluene (8) ${ }^{13} \mathrm{C}$ NMR

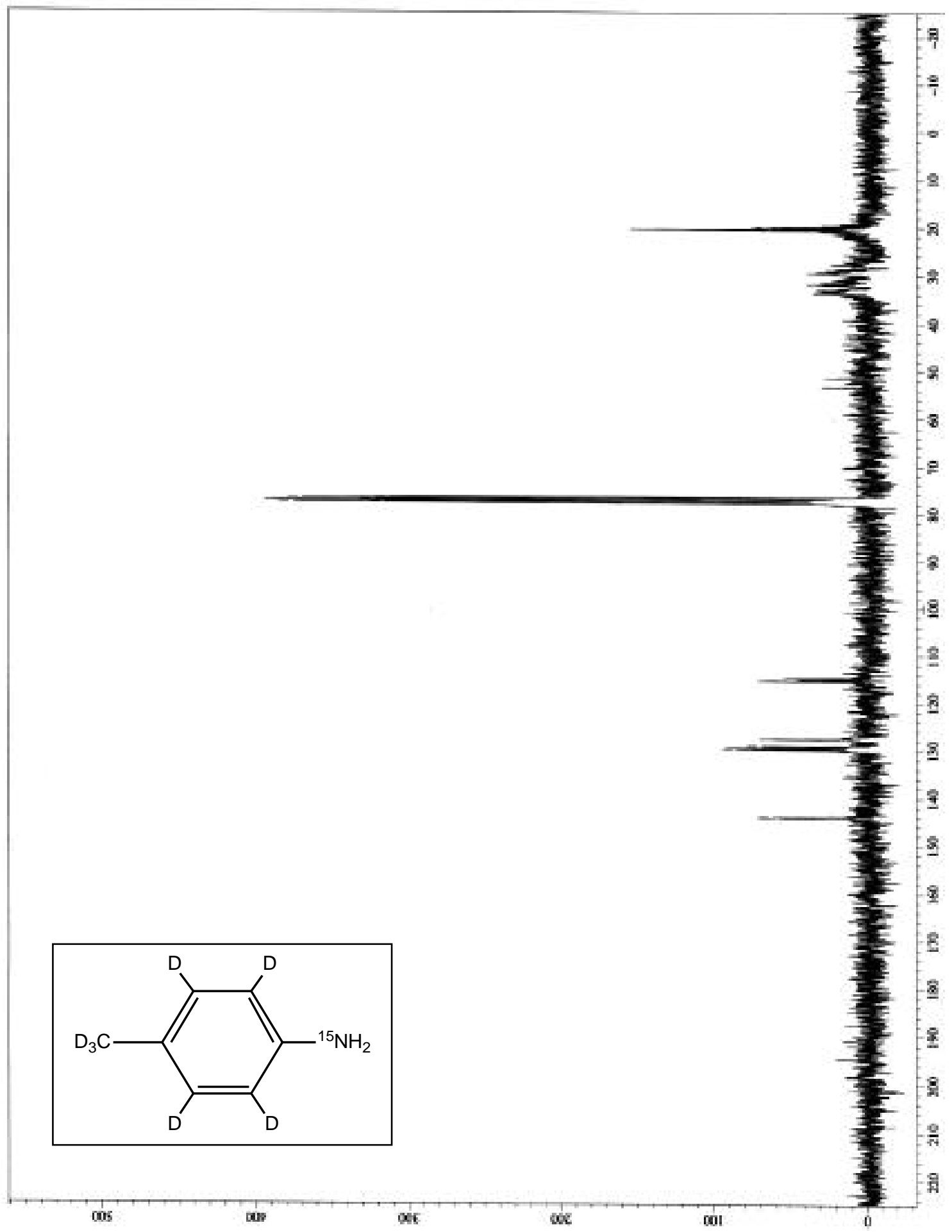


B 4.1 ( $\left({ }^{15} \mathrm{~N}\right)$-N-(4-methylphenyl)-4-methylbenzeneamine (10) ${ }^{1} \mathrm{H}$ NMR

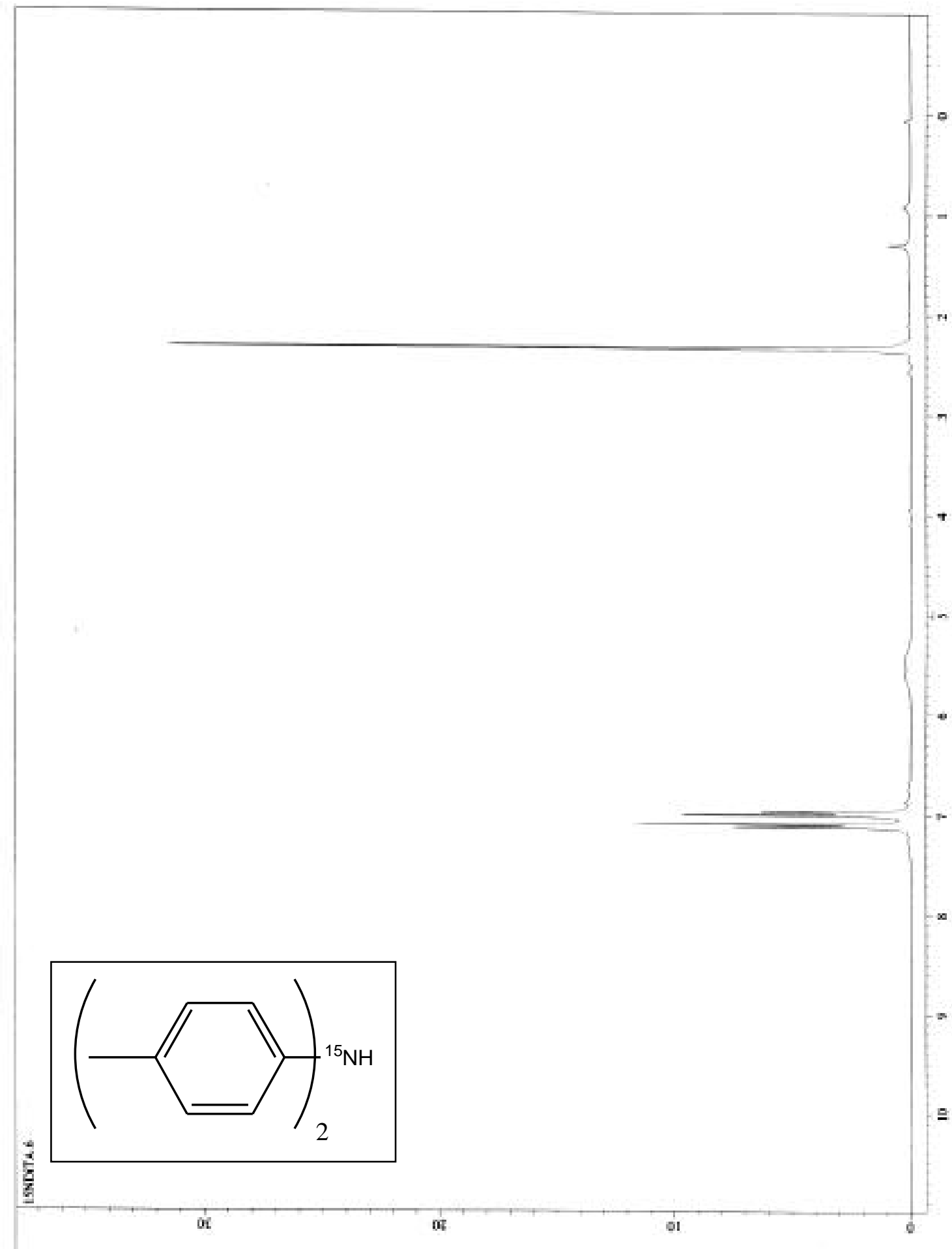


B $4.2\left({ }^{15} \mathrm{~N}\right)-\mathrm{N}$-(4-methylphenyl)-4-methylbenzeneamine (10) ${ }^{13} \mathrm{C}$ NMR

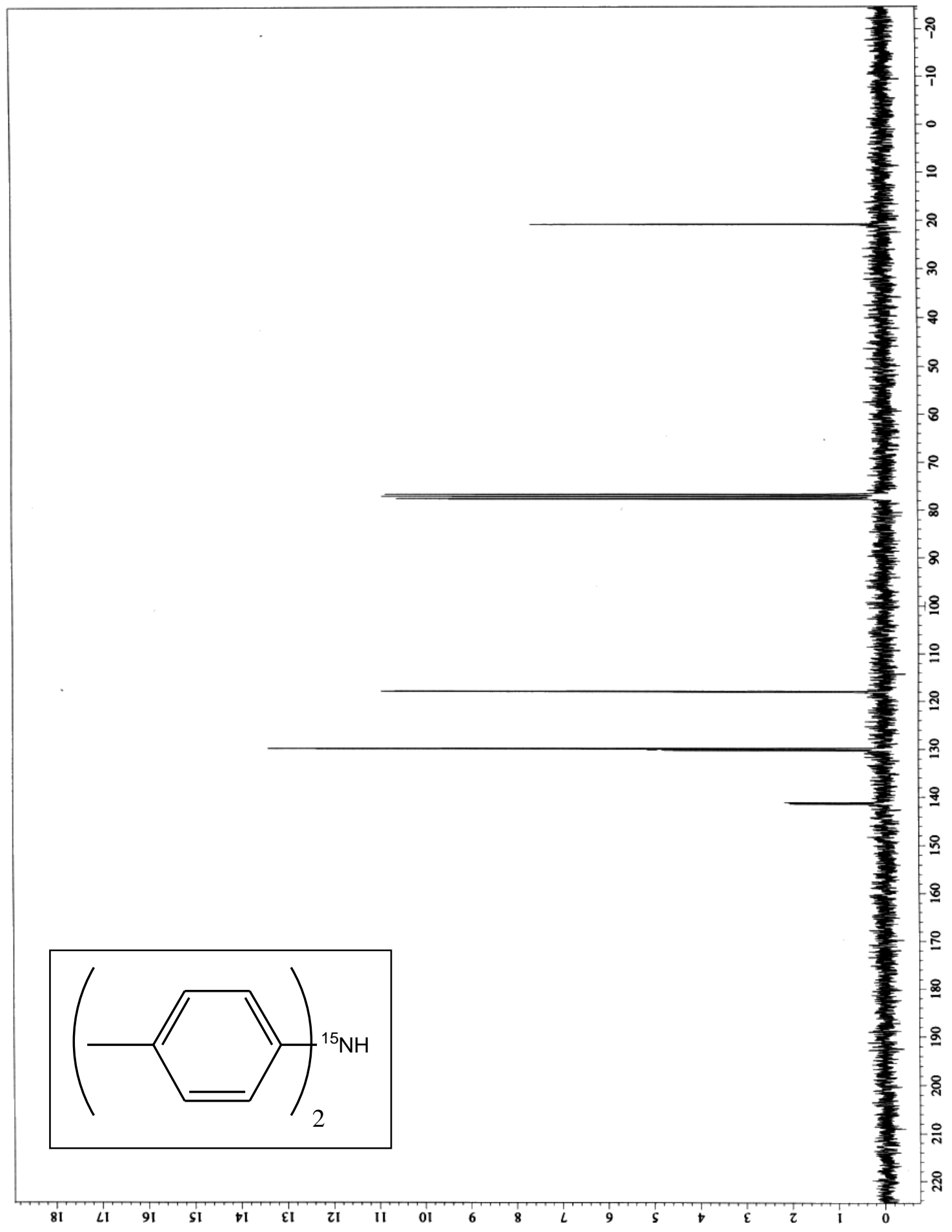


B $4.3\left({ }^{15} \mathrm{~N},{ }^{2} \mathrm{D}_{14}\right) \mathrm{N}$-(4-methylphenyl)-4-methylbenzeneamine (12) ${ }^{1} \mathrm{H}$ NMR

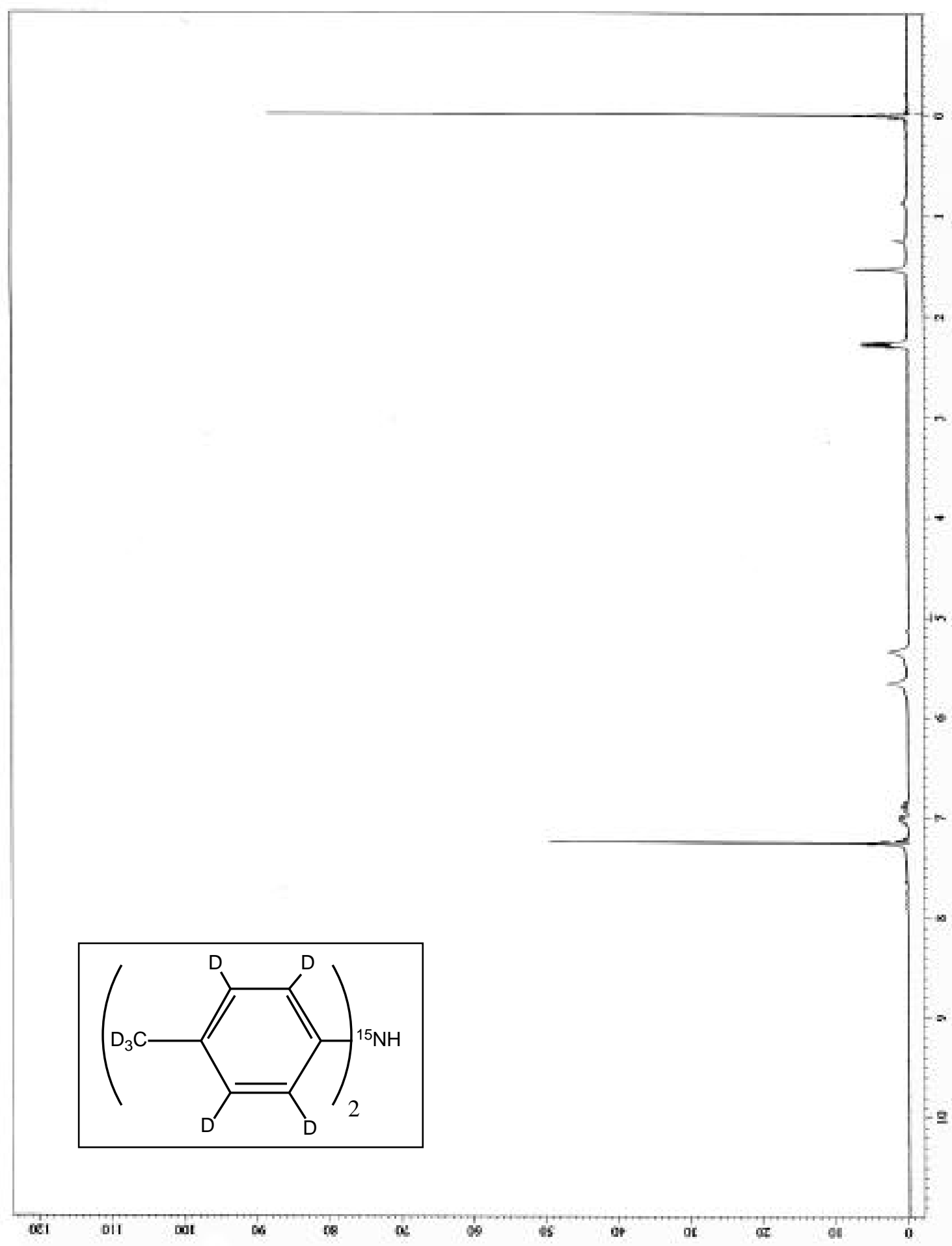


B 4.4 $\left({ }^{15} \mathrm{~N},{ }^{2} \mathrm{D}_{14}\right) \mathrm{N}$-(4-methylphenyl)-4-methylbenzeneamine (12) ${ }^{13} \mathrm{C}$ NMR

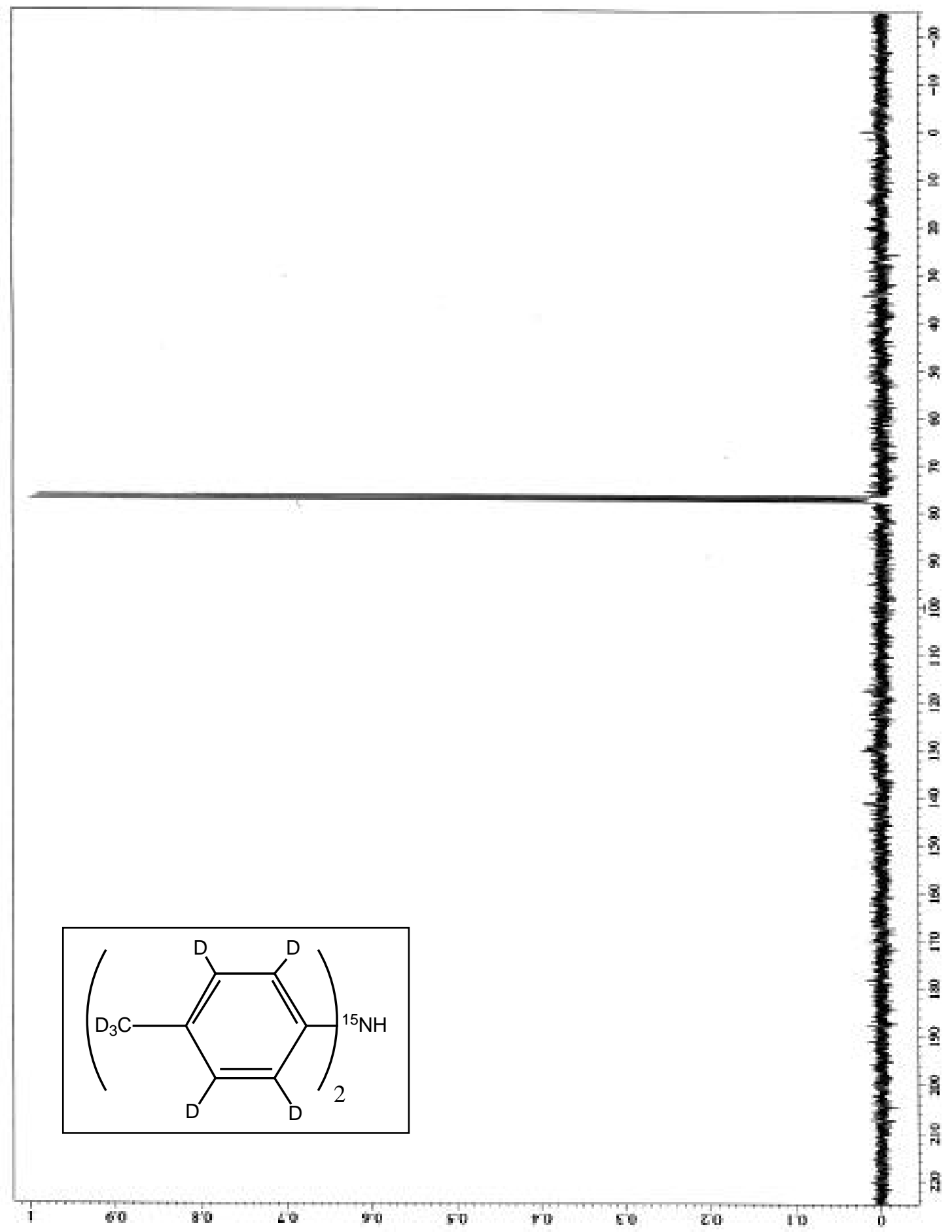




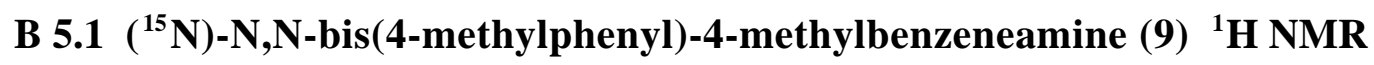

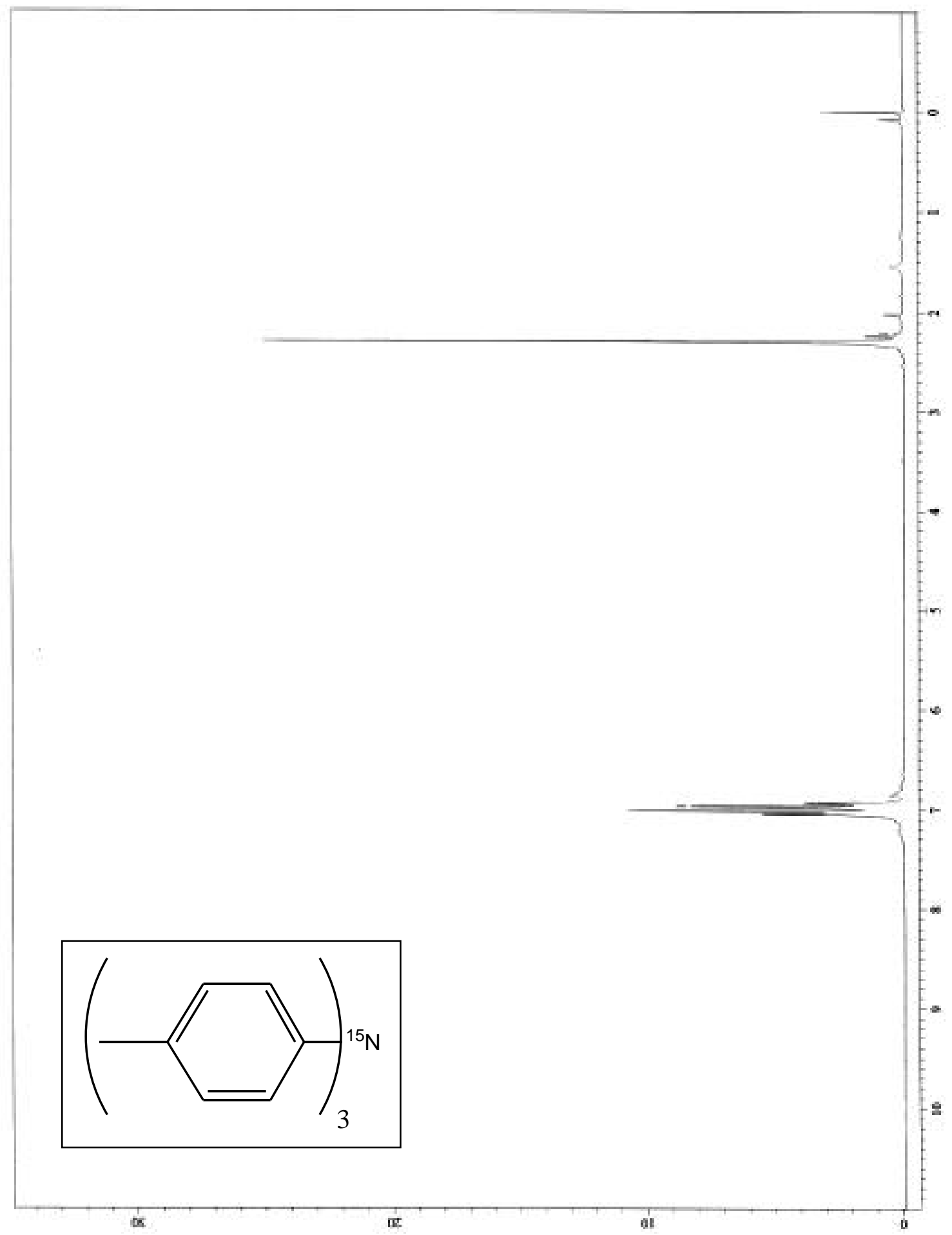


B $5.2\left({ }^{15} \mathrm{~N}\right)$-N,N-bis(4-methylphenyl)-4-methylbenzeneamine $(9){ }^{13} \mathrm{C}$ NMR

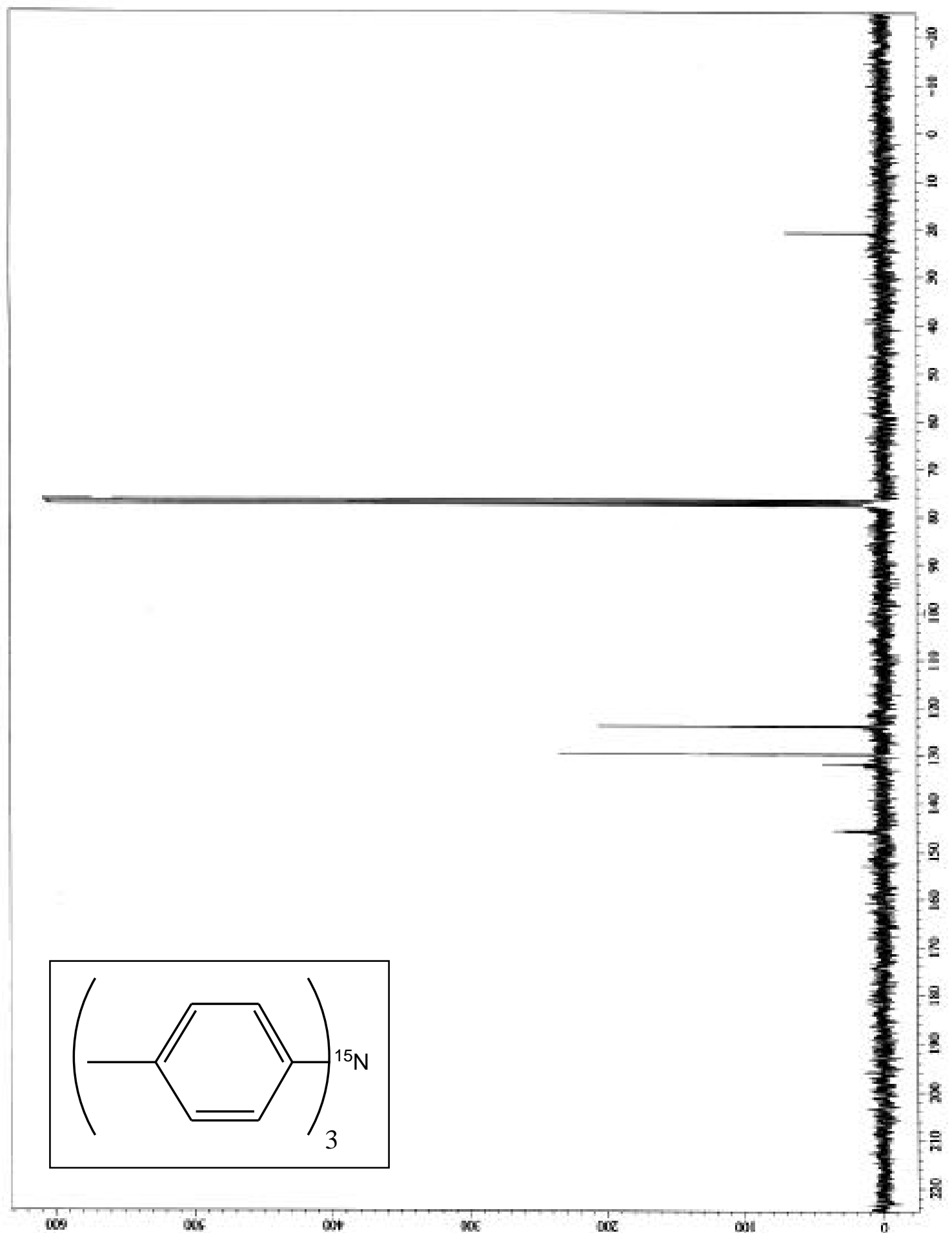


B $5.3\left({ }^{15} \mathrm{~N},{ }^{2} \mathrm{D}_{21}\right)$-N,N-bis(4-methylphenyl)-4-methylbenzeneamine (11)

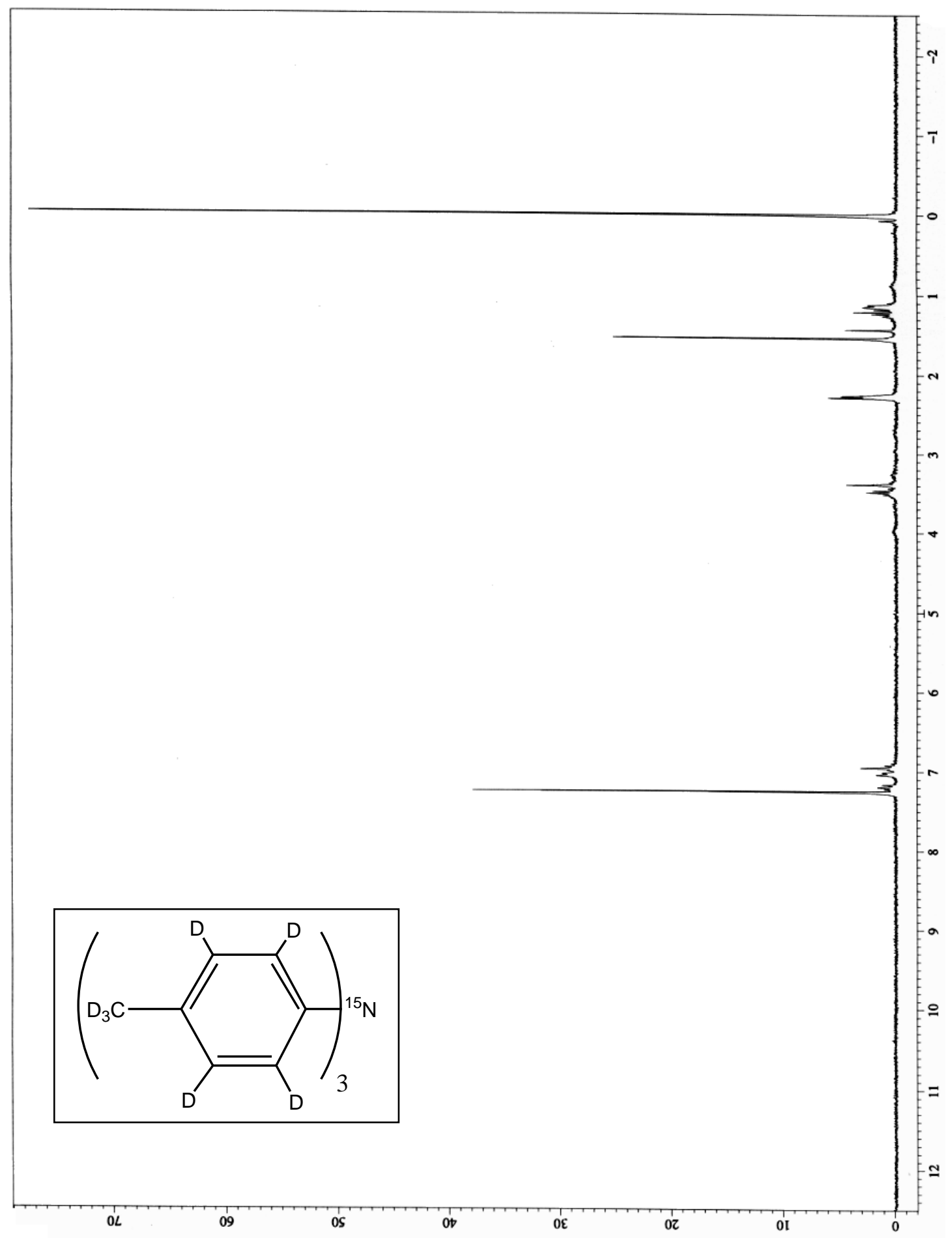


B $5.4\left({ }^{15} \mathrm{~N},{ }^{2} \mathrm{D}_{21}\right)$-N,N-bis(4-methylphenyl)-4-methylbenzeneamine (11) ${ }^{13} \mathrm{C}$ NMR

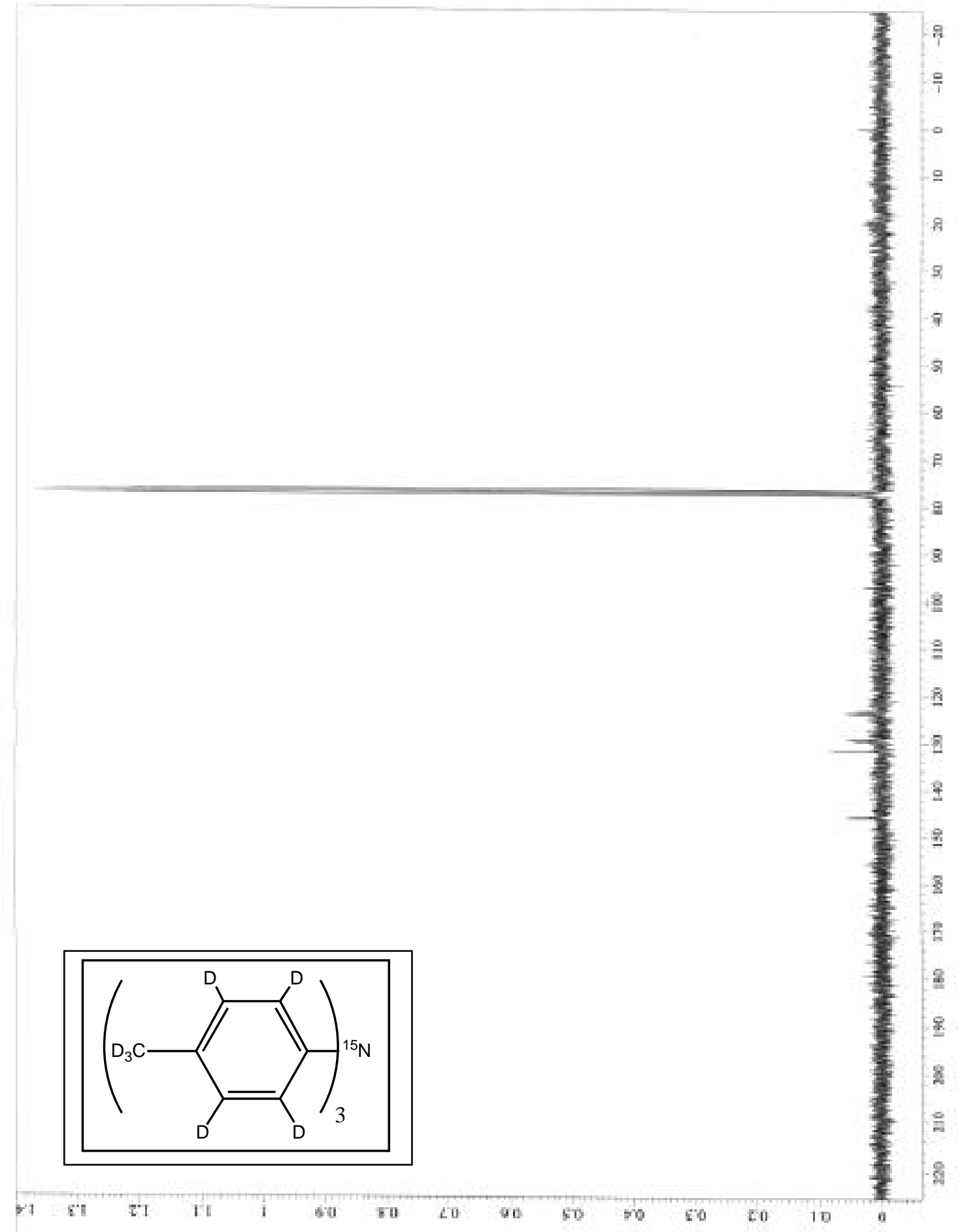


B $5.5 \mathrm{~N}, \mathrm{~N}$-bis(4-methylphenyl)-4-(13CH3)-methylbenzeneamine (13) ${ }^{1} \mathrm{H}$ NMR

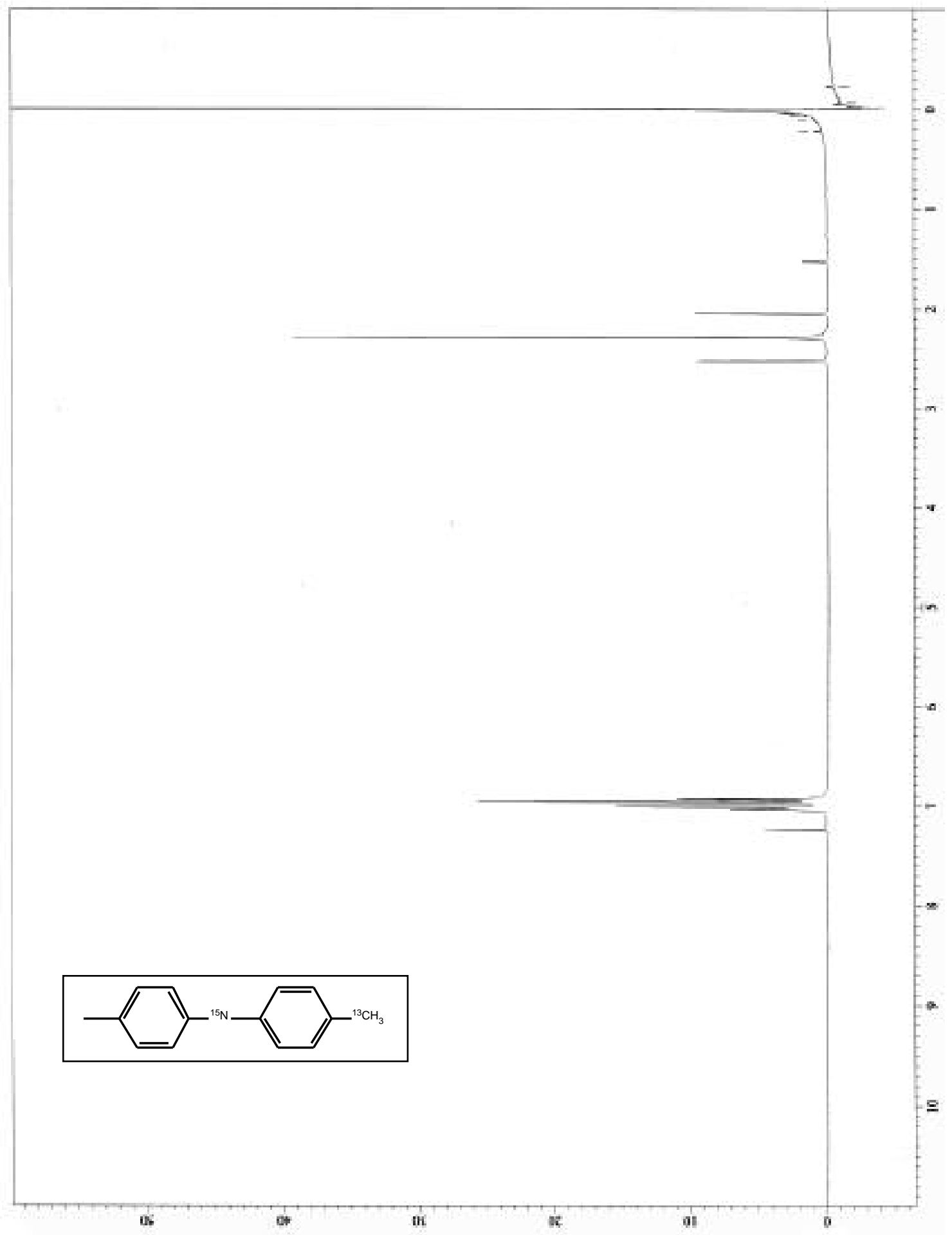


B 5.6 N,N-bis(4-methylphenyl)-4-(13CH3)-methylbenzeneamine (13) ${ }^{13} \mathrm{C}$ NMR

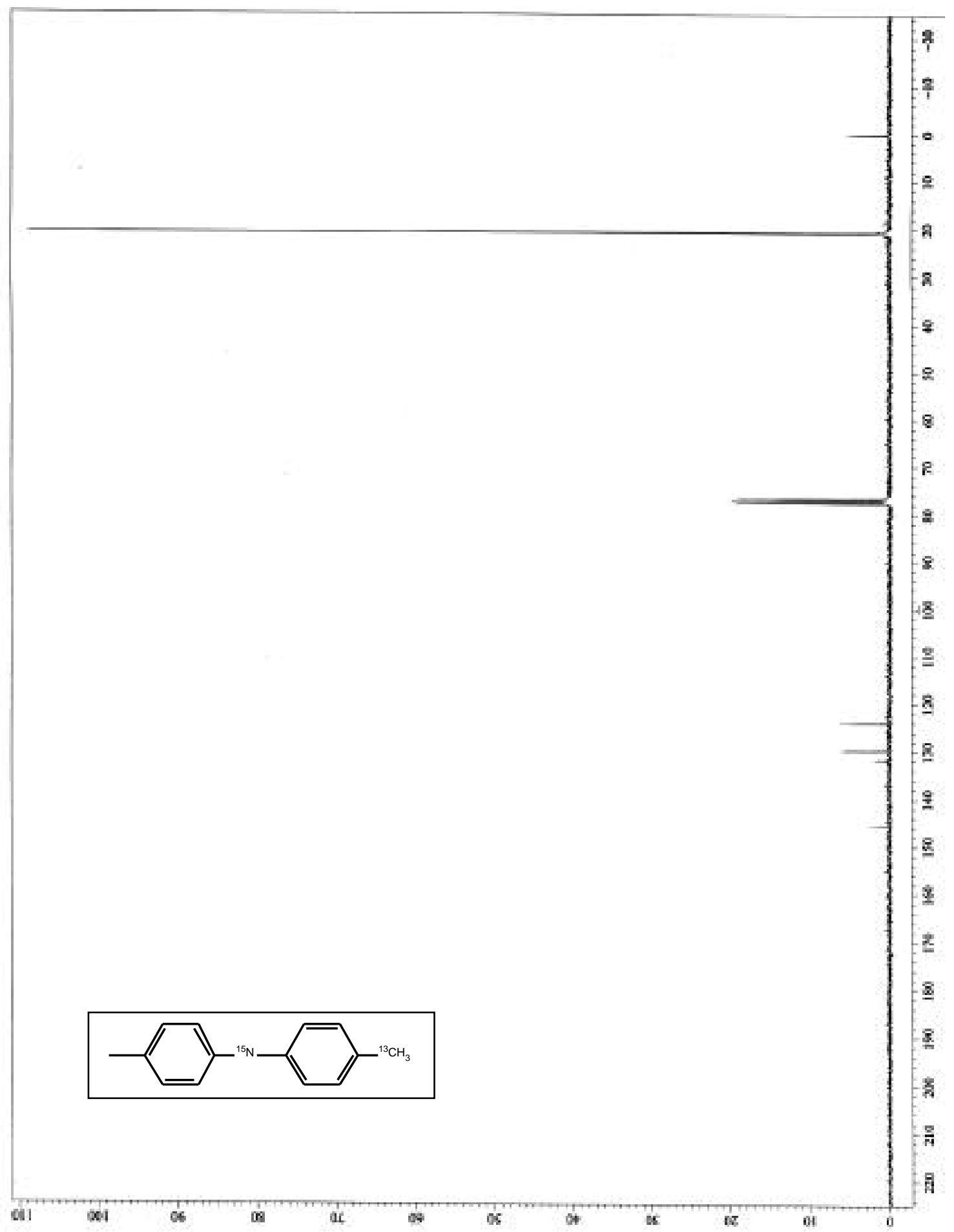




\section{Bibliography}

1 “Organic Photoreceptors for Xerography”, Paul M. Borsenberger and David S. Weiss, Marcel Dekker, Inc, 1998

2 D. M. Pai, B. E. Springett, Rev. Mod. Phys. 65 (1993) 163

3 "Principles of Magnetic Rsonance, $2^{\text {nd }}$ ed", Slichter, C. P., Springer-Verlag: Berlin, 1978

4 "NMR Spectroscopy, $2^{\text {nd }}$ ed", Gunther, Harald, Wiley \& Sons, Chichester, 1995

5 Gullion, T.; Schaefer, J. Advances in Magnetic Resonance (13); Academic Press: New York; Warren, W. S., Ed.; 1989, 57-83.

6 Gullion, T.; Schaefer, J. J. Magn. Reson. 1989, (81), 196.

7 Hewitt, J.M., Henrichs, P.M., Scozzafava, M., Scaringe, R.P., Linder, M., Sorriero, L.J., Macromolecules 17 (1984)2566

8 Smith, K; Bahzad, D.J. Chem. Soc., Chem. Commun. 467-8 (1996)

9 Smith, K.; Musson, A.; DeBoos, G.A. J.Org.Chem. 63: 8448-54 (1998)

10 Kesling, Brian D., Soderberg, Bjorn C., Gullion, Terry, J. Labelled Cpd. Radiopharm. 43, 1059-1068 (2000)

11 Kesling, Brian, Hughes, Eric, Gullion, Terry Solid State Nuclear Magnetic Resonance 16(2000)1-7 